\title{
What life cycle graphs can tell about the evolution of life histories
}

Rueffler Claus

Hans J.A.J. Metz (metz@iiasa.ac.at)

Tom J. M. Van Dooren

\section{Approved by}

Ulf Dieckmann

Director, Evolution and Ecology Program

June 2015 


\title{
What life cycle graphs can tell about the evolution of life histories
}

\author{
Claus Rueffler • Johan A. J. Metz • \\ Tom J. M. Van Dooren
}

Received: 2 December 2010 / Revised: 17 December 2011 / Published online: 5 February 2012

(C) Springer-Verlag 2012

\begin{abstract}
We analyze long-term evolutionary dynamics in a large class of life history models. The model family is characterized by discrete-time population dynamics and a finite number of individual states such that the life cycle can be described in terms of a population projection matrix. We allow an arbitrary number of demographic parameters to be subject to density-dependent population regulation and two or more demographic parameters to be subject to evolutionary change. Our aim is to identify structural features of life cycles and modes of population regulation that correspond to specific evolutionary dynamics. Our derivations are based on a fitness proxy that is an algebraically simple function of loops within the life cycle. This allows us to phrase the results in terms of properties of such loops which are readily interpreted biologically. The following results could be obtained. First, we give sufficient conditions for the
\end{abstract}

C. Rueffler $(\square)$

Mathematics and Biosciences Group, Department of Mathematics, University of Vienna,

Nordbergstrasse 15, 1090 Vienna, Austria

e-mail: claus.rueffler@univie.ac.at

J. A. J. Metz

Mathematical Institute and Institute of Biology, Leiden University,

P. O. Box 9512, 2300 RA Leiden, The Netherlands

e-mail: j.a.j.metz@biology.leidenuniv.nl

J. A. J. Metz

Evolution and Ecology Program, International Institute of Applied Systems Analysis,

2361 Laxenburg, Austria

J. A. J. Metz · T. J. M. Van Dooren

Netherlands Centre for Biodiversity, Naturalis, P. O. Box 9517, 2300 RA Leiden, The Netherlands

T. J. M. Van Dooren

UMR 7625 Ecology and Evolution, Eco-Evolutionary Mathematics,

Ecole Normale Supérieure, Rue d'Ulm 46, 75230 Paris Cedex 05, France

e-mail: vandoore@biologie.ens.fr 
existence of optimisation principles in models with an arbitrary number of evolving traits. These models are then classified with respect to their appropriate optimisation principle. Second, under the assumption of just two evolving traits we identify structural features of the life cycle that determine whether equilibria of the monomorphic adaptive dynamics (evolutionarily singular points) correspond to fitness minima or maxima. Third, for one class of frequency-dependent models, where optimisation is not possible, we present sufficient conditions that allow classifying singular points in terms of the curvature of the trade-off curve. Throughout the article we illustrate the utility of our framework with a variety of examples.

Keywords Adaptive dynamics - Density dependence - Frequency dependence . Life history theory $\cdot$ Matrix model $\cdot$ Evolutionary optimisation

\section{Mathematics Subject Classification (2000) 92D15}

\section{Introduction}

Life history theory aims at explaining the differences in rates of growth, maturation and reproduction between species and populations as a result of natural selection (e.g. Stearns 1992; Charnov 1993; Charlesworth 1994; Roff 2002). Time and energy are necessarily limiting factors for the long-term growth of any population, and life history theory revolves around the question how these limiting factors are optimally allocated to the different processes within a life cycle. The concern about the allocation of limiting factors leads us directly to the concept of a trade-off which is crucial to life history theory. The idea is that different demographic parameters that affect fitness cannot evolve independently but are developmentally or physiologically coupled. A change in one parameter that increases fitness has to be accompanied by changes in one or more other parameters with detrimental effects. Life history theory aims at predicting which compromises are favored by natural selection under different ecological and developmental boundary conditions (e.g. Stearns 1992; Charlesworth 1994; Charnov 1993; Roff 2002).

An influential method to predict life history patterns has been introduced by Levins (1962, 1968). His method is an optimisation procedure that can be applied when only two demographic parameters are evolving and can be visualized geometrically (Fig. 1). First one computes a fitness measure for every combination of demographic parameters resulting in a two-dimensional fitness landscape. In a second step, one chooses which parameter combinations are admissible, that is, which combinations are assumed to be possible given developmental and physiological processes. For two parameters the boundary of the set of admissible combinations can be depicted in the form of a trade-off curve. If fitness is increasing in both parameters under study, the expected evolutionary outcome based on Levins' approach is given by the combination of parameters corresponding to the point on the trade-off curve that lies on the highest fitness contour touching the trade-off curve (Fig. 1). The exact prediction depends on the curvatures of both the trade-off and the contour lines of the fitness landscape. While the former curve is a choice of the modeler that is ideally informed by 

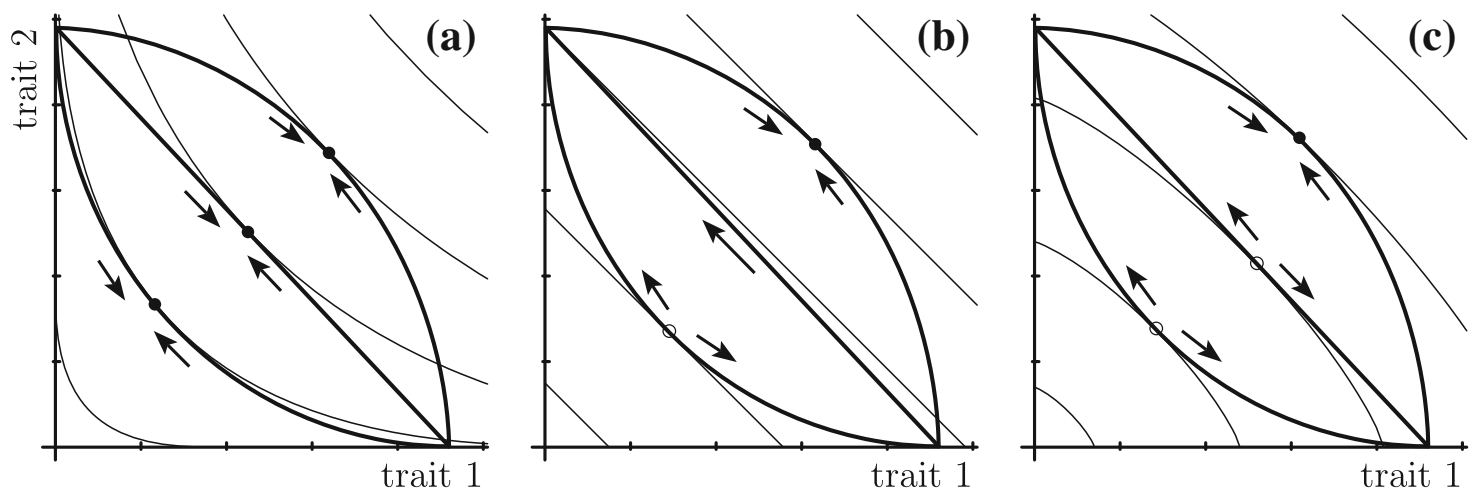

Fig. 1 Levins' geometrical fitness set approach. Thin lines represent the contour lines of three hypothetical fixed fitness landscapes, each increasing in both traits. Thick lines correspond to different realizations of a trade-off curve. If fitness is increasing in the two demographic parameters considered evolvable, selection pushes a population's trait distribution towards a specific trade-off curve. From then on, trait combinations can be assumed to lie on the trade-off curve. Selection acts to increase fitness and changes the combination of trait values as indicated by the arrows. Circles indicate intermediate trait combinations where the fitness contour is tangent to the trade-off curve. Such points are either fitness maxima (filled circles) or fitness minima (open circles). a Convex fitness contour lines. b Linear fitness contour lines. c Concave fitness contour lines

knowledge about developmental and physiological constraints in the specific organism under study, the latter is a property of the algebraic nature of the relationship between the fitness measure and the demographic parameters considered evolvable. In the simplest case where two evolving parameters affect fitness linearly, contour lines are straight lines (Fig. 1b) and the following conclusions can be drawn: In case of convex trade-offs selection results in extreme phenotypes that derive their fitness from a high value of one demographic parameter. In case of concave trade-offs selection results in intermediate phenotypes that derive their fitness from a combination of intermediate values of demographic parameters.

Since Levins' methodology is based on the idea that natural selection maximizes a fitness measure, the results will depend on the chosen fitness measure. In the last decades it became clear that an appropriate choice depends on the assumptions made about the ecology and that the choice has to be based on an invasion argument (Metz et al. 1992, 1996b, 2008a,b; Mylius and Diekmann 1995; Pásztor et al. 1996). Specifically, it has been shown that the details of population regulation influence whether well-known quantities like the intrinsic growth rate $r$, the basic reproduction ratio $R_{0}$ or other quantities with a less straightforward interpretation should be maximized to arrive at the same predictions as derived from an evolutionary invasion analysis. More severely, most instances of population regulation preclude the existence of an optimisation principle altogether and an invasion analysis is the only method to arrive at evolutionary predictions that are consistent with the assumptions made about the ecology (Metz et al. 1996b, 2008a,b). When the latter is the case we call selection frequency-dependent (Heino et al. 1998).

From the previous considerations it becomes clear that the nature of population regulation, curvature properties of the fitness landscape and the trade-off curve are key determinants of the evolutionary dynamics. To illustrate this point further, we sketch a life history problem analyzed by Takada and Nakajima (1996). Many herbaceous 


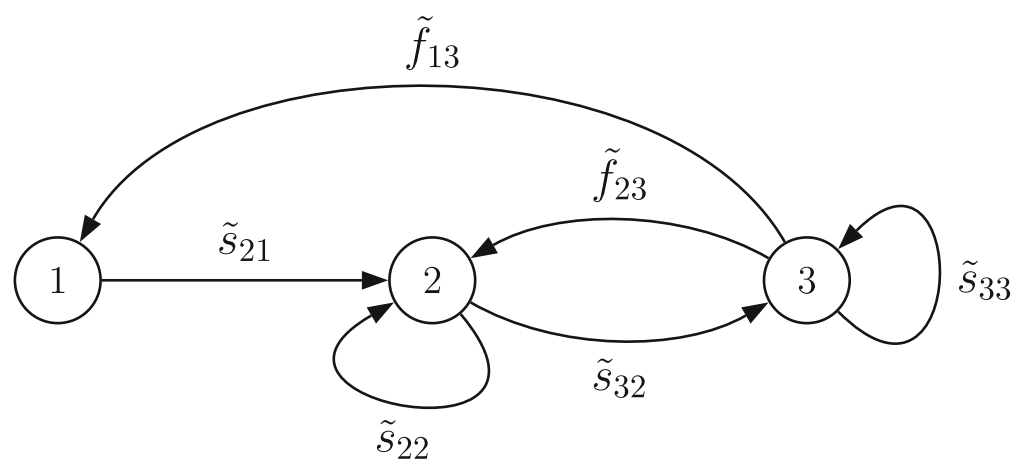

Fig. 2 Life cycle graph of a hypothetical perennial plant with a seedling (1), juvenile (2) and flowering state (3)

plants have the ability to reproduce sexually via seeds and asexually via vegetative propagules. What is the optimal resource allocation scheme for these two modes of reproduction? Takada and Nakajima (1996) formulated this question as a decision between either investing resources in many small offspring with low survival probability (seeds) or few large offspring with high survival probability (vegetative propagules). To analyze this question, Takada and Nakajima classified individuals as seedlings, juveniles or plants in the flowering stage, as shown in the corresponding life cycle graph in Fig. 2. Per year, seedlings turn into juveniles with probability $\tilde{s}_{21}$ and juveniles turn into flowering plants with probability $\tilde{s}_{32}$. Juvenile and flowering plants remain in their current state with probability $\tilde{s}_{22}$ and $\tilde{s}_{33}$, respectively. Flowering plants can reproduce via seeds, resulting in $\tilde{f}_{13}$ seedlings, or via vegetative propagules, resulting in $\tilde{f}_{23}$ juvenile plants in the next season. Takada and Nakajima assume a trade-off between $\tilde{f}_{13}$ and $\tilde{f}_{23}$, that is, an increase in one of these parameters is accompanied by a decrease in the other and vice versa. Furthermore, they assume that seedling survival and the production of seeds decrease with increasing population density, reflecting intraspecific competition. More specifically, $\tilde{f}_{13}$ and $\tilde{s}_{21}$ are assumed to be monotonically decreasing functions of the total population density given by $N_{1}+N_{2}+N_{3}$. For this specific model Takada and Nakajima derive that selection favors the combination of $\tilde{f}_{13}$ and $\tilde{f}_{23}$ maximizing the total population size. In accordance with Levins' results, in case of concave trade-offs the optimisation principle has a maximum at an intermediate value of $\tilde{f}_{13}$ so that vegetative and seed reproduction exist next to each other, while in case of convex trade-offs the optimisation principle has a maximum at the upper limit of either $\tilde{f}_{13}$ or $\tilde{f}_{23}$ and all resources are expected to be invested in one mode of reproduction. A natural question to ask is how robust this result is when the specific assumptions of Takada and Nakajima are modified? For example, is optimisation still possible when survival of vegetative propagules is density-dependent instead of the production of seeds? Does the answer depend on whether survival of seeds and vegetative propagules decrease with the same or with different population statistics? And how do all these results change if we assume that the survival probability of adult plants rather than the number of vegetative propagules trades off with seed production?

To search for patterns in the relationship between assumptions and evolutionary outcomes one could analyze many model variants separately. Alternatively, one searches for structural model features that are causally linked with specific evolutionary 
outcomes. The advantage of the latter strategy is at least twofold. First, once the work is done, one can predict the evolutionary dynamics in a large class of models. Second, identifying causal relationships is the essential step towards a true understanding of observed phenomena. In this article we follow this second approach. As a model family we choose a discrete time structured population framework represented by population projection matrices (Caswell 2001). Thus, we only consider life histories that can be described with a finite number of states. We allow for an arbitrary number of demographic parameters to be subject to evolutionary change although certain results could only be obtained for the case of two evolving parameters. Finally, an arbitrary number of demographic parameters can be affected by density-dependent population regulation whereby parameters can be regulated by specific combinations of the densities of individuals in different states.

Since this article is rather comprehensive and not necessarily meant to be read from "cover to cover" we here give a detailed overview of its structure. Section 2 contains preliminaries and consists of six subsections. In Sect. 2.1 we delineate the considered model family. In Sect. 2.2 we present an algebraically simple proxy for invasion fitness that forms the backbone of all further derivations. In Sect. 2.3 we review results from the theory of Adaptive Dynamics, an approximate description of evolutionary dynamics technically based on the assumption that evolution is mutation limited. In Sect. 2.4 we summarize the necessary and sufficient conditions for the existence of an evolutionary optimisation principle derived by Metz et al. (1996b, 2008a) and introduce the notion of frequency-dependent selection. In Sect. 2.5 we review the theory of invasion boundaries, special contour lines of the fitness landscape that partition the trait space into phenotypes that are or are not able to invade a given resident community. In Sect. 2.6 we consider the limitations of our approach and more in particular give a brief outlook on models with diploid sexual reproduction. The following four sections contain our results. In Sect. 3 we derive various conditions that allow for a pure optimisation approach. In its first part (Sect. 3.1) we give conditions that are sufficient for the existence of an optimisation principle derived from the aforementioned fitness proxy. In Sect. 3.2 we present an extensive list of such optimisation principles. In Sect. 3.3 we collect and extend three classical optimisation principles, based on the abundance of a single limiting resource, on the dominant eigenvalue of the population projection matrix $\lambda_{\mathrm{d}}$, and on the basic reproduction ratio $R_{0}$, respectively. In Sect. 4 we deal with models where no optimisation principle exists and state necessary conditions for selection to be frequency-dependent. In Sect. 5 we derive conditions in terms of properties of loops in the life cycle graph that correspond to invasion boundaries that are straight lines, convex or concave. This allows determining the range of trade-off curvatures for which singular points are invadable or uninvadable. In Sect. 5.1 we do so under the restriction that only two demographic parameters are evolving and in Sect. 5.2 we do the same for an arbitrary number of evolving parameters. While the results for two-dimensional trait spaces are rather general, the extension to higher dimensional trait spaces only applies to models with a high degree of symmetry. In Sect. 6 we take a closer look at a subclass of models with frequency-dependent selection. Given that fitness contours are straight lines and that population regulation is of a particularly simple kind we are able to classify singular points as a function of the trade-off curvature. The material is organized such that the first three results 
sections (3 Optimisation, 4 Frequency-Dependent Selection and 5 Curvature of Invasion Boundaries) can be read independently of each other. Only the last results section (6 Frequency-Dependent Selection and Linear Invasion Boundaries) draws on preceding results. We finish by applying our toolbox to various life history problems based on the life cycle of Fig. 2 (Sect. 7).

\section{Preliminaries}

\subsection{The life cycle and population dynamics}

We restrict ourselves to life cycles that can be described with $n$ discrete i-states (i for individual, see Metz and Diekmann (1986)) in a discrete time framework. Furthermore, we proceed as if individuals reproduce clonally; our results extend to Mendelian populations under conditions specified in Sect. 2.6. The population dynamics of a clone can be described by

$$
N_{t+1}=\mathrm{A} \boldsymbol{N}_{t}
$$

where $\mathrm{A}=\left[\tilde{a}_{l k}\right]$ is the $n \times n$ non-negative population projection matrix corresponding to a specific life cycle and $N_{t}=\left(N_{1 t}, \ldots, N_{n t}\right)^{\mathrm{T}}$ is the vector of densities in the different i-states at time $t$ (Caswell 2001). Census is taken just before reproduction. At reproduction individuals in i-state $k$ produce offspring of which $\tilde{f}_{l k} \geq 0$ are alive at the next census and in i-state $l$. After the reproduction episode all individuals - other than the newborns - undergo one of the following state-transitions: (i) with probability $\tilde{s}_{k k}$ they stay in their current i-state $k$, (ii) with probability $\tilde{s}_{l k}$ they move from i-state $k$ to i-state $l(l \neq k)$, (iii) with probability $\tilde{d}_{k}=1-\sum_{l} \tilde{s}_{l k}$ they die. Thus, A can be decomposed into a fertility matrix $\mathrm{F}=\left[\tilde{f}_{l k}\right]$ and a state-transition matrix $\mathrm{S}=\left[\tilde{s}_{l k}\right]$ such that $\mathrm{A}=\mathrm{F}+\mathrm{S}$ (Caswell 2001, p. 110). We will refer to $\tilde{f}_{l k}$ and $\tilde{s}_{l k}$ as demographic parameters. Throughout this article a tilde on a demographic parameter indicates that it is not necessarily constant on the ecological time scale but can be under density dependent population regulation. The described framework includes cases with i-states such as age class, size class, developmental state, or, in a patch model, spatial location. Individuals can either occur in one i-state at birth (e.g. immature, or small) or in different i-states (e.g., births in different patches). We will refer to clones with different demographic parameters as types and denote the population vector of the $j$ th type with $N^{j}$. We refer to the vector of densities of $m$ coexisting types $\mathcal{N}_{t}=\left(\boldsymbol{N}_{t}^{1}, \ldots, \boldsymbol{N}_{t}^{m}\right) \in \mathscr{N}=\left(\mathbb{R}_{+}^{n}\right)^{m}$ as population- or p-state.

Demographic parameters $\tilde{s}_{l k}$ and $\tilde{f}_{l k}$ give the state-transition probability and fertility, respectively, as they would be measured in the field or in the lab at a particular point in time. We assume that demographic parameters consist of a hereditary component - defined as the value in a 'virgin' environment, i.e., in the absence of any conspecifics - that is modified through the effect of conspecifics, either directly or indirectly due to their influence on the environment. We denote the hereditary component of $\tilde{s}_{l k}$ and $\tilde{f}_{l k}$ by $s_{l k}$ and $f_{l k}$, respectively. Evolutionary change occurs through mutations affecting hereditary components. In principle all hereditary components may mutate. 
However, we will often assume that only a subset of hereditary components is evolving and we refer to these as traits. The traits of the $j$ th type will be collected in the vector $\boldsymbol{x}^{j}=\left(x_{1}^{j}, x_{2}^{j}, \ldots\right)$. The phenotypic composition of a population of $m$ types is given by $\mathcal{X}=\left(\boldsymbol{x}^{1}, \ldots, \boldsymbol{x}^{m}\right) \in \mathscr{X}=\left((0,1)^{n_{\mathrm{s}}} \times \mathbb{R}_{+}^{n_{\mathrm{f}}}\right)^{m}$ with $n_{\mathrm{s}}$ the number of evolving state-transition parameters and $n_{\mathrm{f}}$ the number of evolving fertility parameters. We will often distinguish between trait values of a common resident type and a rare mutant type by labeling the latter with a prime: $\boldsymbol{x}^{\prime}=\left(x_{1}^{\prime}, x_{2}^{\prime}, \ldots\right)$. In principle, the values of the various traits can occur in any combination as long as for any i-state $k$ the biologically inevitable constraint $\sum_{l} s_{l k} \leq 1$ is fulfilled. Note that we implicitly assume that whenever one or more state-transition probabilities $s_{l k}$ evolve, this includes a change in the death probability $d_{k}$ such that the constraint $1=d_{k}+\sum_{l} s_{l k}$ remains fulfilled.

Since the dominant eigenvalue of a non-negative matrix and hence, long term population growth, is an increasing function of all demographic parameters (e.g. Horn and Johnson 1985), we can expect that selection acts to increase all traits. However, no organism is immortal or can produce infinitely many offspring. Thus, constraints that prevent an indefinite simultaneous increase of all traits have to exist and restrict the set of possible phenotypes. We will refer to this set as feasibility set and assume that it is bounded by the surfaces of the positive cone of $\mathbb{R}^{n_{\mathrm{s}}+n_{\mathrm{f}}}$ where one trait is zero and the other nonnegative and a smooth $\left(n_{\mathrm{S}}+n_{\mathrm{f}}-1\right)$-dimensional constraint manifold that divides the positive cone of $\mathbb{R}^{n_{\mathrm{s}}+n_{\mathrm{f}}}$ into an inward part adjacent to the origin, the feasibility set, and an outward part. Once the constraint manifold has been reached, an increase in one trait can only be bought at the expense of a decrease in other traits. In two-dimensional trait spaces this idea is commonly depicted as a trade-off curve: the set of trait vectors $\left(x_{1}, x_{2}\right)$ that constitute the non-trivial boundary of the feasibility set corresponds to a one-dimensional trade-off curve $\left\{\left(x_{1}, x_{2}\right) \mid x_{2}=T\left(x_{1}\right)\right\}$ with $\mathrm{d} T / \mathrm{d} x_{1}<0$ (Fig. 1).

If the matrix entries $\tilde{a}_{l k}$ would be independent of the population densities $N_{t}$, i.e., when all demographic parameters equal their hereditary component, then Eq. (1) is a linear model resulting in exponential growth. With increasing density, however, individuals are expected increasingly to exert negative influences on each other, either through direct interference such as fighting or mediated by resources, predators or pathogens. This prevents long-term exponential growth from occurring. Following Diekmann, Metz and others (Metz and Diekmann 1986; Metz et al. 1992; Diekmann et al. 1998, 2001, 2003; Diekmann 2004; Meszéna et al. 2006), dependence among individuals, hence, nonlinearities, can be accounted for in a two-step procedure:

(i) Demographic parameters may depend on components of the environment. These components can be determined by the requirement that individuals become independent of each other and hence Eq. (1) becomes linear in $\boldsymbol{N}_{t}$ whenever the environment is given as a function of time. Examples of such components are abundance of resources, predators, pathogens, or, if interactions occur directly between individuals, costs due to interference.

(ii) The components of the environment, in turn, are determined by the individuals that constitute the population, for example through consumption of resources, by making contacts with other individuals where pathogens can be transmitted, or through fighting. 
Thus, by appropriately defining the environment, all interactions are mediated by components of the environment. These components are on the one hand determined by the population composition as described by $\mathcal{N}_{t}$ and $\mathcal{X}$ and on the other hand affect demographic parameters. As intermediary in this environmental feedback loop we introduce the function $\boldsymbol{E}: \mathscr{N} \times \mathscr{X} \rightarrow \mathscr{E}=\mathbb{R}_{+}^{k}$ that accounts for all density- and frequency-dependent effects that arise through direct and indirect interactions between the individuals in the population. Thus, we assume that the relevant environmental components can be described by $k$ scalars $E_{j}\left(\mathcal{N}_{t}, \mathcal{X}\right)$ which we collect in a vector $\boldsymbol{E}\left(\mathcal{N}_{t}, \mathcal{X}\right)$ to which we refer as feedback environment. Doing the bookkeeping of interactions via the intermediate step of environmental components has two advantages. First, many results get a mechanistic biological flavor since they can be re-phrased in terms of e.g. the abundance of resources, predators or pathogens. Second, the number of environmental coordinates is an upper limit for the number of possibly coexisting species (Levin 1970; Diekmann et al. 2003; Meszéna et al. 2006).

In many scenarios $\boldsymbol{E}\left(\mathcal{N}_{t}, \mathcal{X}\right)$ is determined by weighted sums of densities of individuals in the $n$ different $\mathrm{i}$-states, each summed over all $m$ types present in the population. The weighting factors reflect the differential impact of individuals in different $\mathrm{i}$-states on the various limiting factors and may or may not depend on the trait values. Then the feedback environment is specified by

$$
\boldsymbol{E}\left(\mathcal{N}_{t}, \boldsymbol{\mathcal { X }}\right)=F\left(\sum_{i=1}^{m} \sum_{j=1}^{n} \nu_{1 j}\left(\boldsymbol{x}^{i}\right) N_{j t}^{i}, \sum_{i=1}^{m} \sum_{j=1}^{n} \nu_{2 j}\left(\boldsymbol{x}^{i}\right) N_{j t}^{i}, \ldots\right)
$$

where $F$ is a vector-valued function and the functions $v_{k j}$, specifying the weights, are positive and scalar-valued.

How does the feedback environment $\boldsymbol{E}\left(\mathcal{N}_{t}, \mathcal{X}\right)$ enter Eq. (1) describing the population dynamics? Here we assume that the realized value of any given demographic parameter at time $t$ is the product of its value as it could be measured in the absence of any effects due to the presence of other individuals and a scalar-valued regulatory function $R_{\mathrm{z}, l k}: \mathscr{E} \rightarrow[0,1]$. This function has two specifiers: $\mathrm{z} \in\{\mathrm{s}, \mathrm{f}\}$ indicates whether the regulated demographic parameter is a state-transition or a fertility parameter and $k, l \in\{1, \ldots, n\}$ indicate the position of the demographic parameter in the matrix $A$. We denote the value of a demographic parameter in the absence of interactions with conspecifics with $z_{l k}$. The realized value at time $t$, given $\mathcal{N}_{t}$ and $\mathcal{X}$, thus equals

$$
\tilde{z}_{l k}:=z_{l k} R_{\mathrm{z}, l k}\left(\boldsymbol{E}\left(\mathcal{N}_{t}, \mathcal{X}\right)\right)
$$

We exclude the possibility of Allee-effects and assume that the presence of other individuals, be it of the own type or of other types, always has a detrimental effect. Hence, $R_{\mathrm{z}, l k}$ has range $[0,1]$ and $z_{l k, t} R_{\mathrm{z}, l k}\left(\boldsymbol{E}\left(\mathcal{N}_{t}, \mathcal{X}\right)\right) \leq z_{l k}$. Furthermore, all regulatory functions $R_{\mathrm{z}, l k}$ are assumed to be increasing in each coordinate $E_{j}\left(\mathcal{N}_{t}, \mathcal{X}\right)$ of $\boldsymbol{E}\left(\mathcal{N}_{t}, \mathcal{X}\right)$, reflecting that an increasing number of resources has a positive effect on population growth. For coordinates of $\boldsymbol{E}\left(\mathcal{N}_{t}, \mathcal{X}\right)$ that reflect predators or parasites is natural to assume that the functions $R_{\mathrm{z}, l k}$ are decreasing in such coordinates since predators and parasites have a negative effect on population growth. Thus, such coor- 
dinates have to be redefined by, for example, subtracting them from their maximal value. We make one further assumption about population regulation, to which we will refer as separability. By this we mean that a regulatory function becomes independent of the trait vector $\boldsymbol{x}^{j}$ of a specific type if the density of that type equals zero, $\boldsymbol{N}_{t}^{j}=0$. This means in particular that the realized value of a regulated trait of a rare mutant type is the product of its value in the absence of interactions with conspecifics, $x_{l k}^{\prime}$, and the value of a regulatory function $R_{\mathrm{z}, l k}$ that effectively only depends on the traits $\mathcal{X}$ and densities $\mathcal{N}_{t}$ of the resident types: $z_{l k, t}^{\prime} R_{\mathrm{z}, l k}\left(\boldsymbol{E}\left(\mathcal{N}_{t}, \mathcal{X}\right)\right)$. In other words, the effect of the common resident types on a rare mutant type do not depend on the trait-values of the mutant. The separability assumption is fulfilled if $\boldsymbol{E}\left(\mathcal{N}_{t}, \mathcal{X}\right)$ is of the form of Eq. (2). Separability is expected when the effect of different types on each other is mediated through the abundance of finitely many types of resources, predators and parasites. An example where the separability assumption is not fulfilled is size-dependent competition where the effect of different types on each other is a direct function of their size-difference (c.f. MacArthur 1970).

We assume that population regulation results in a unique nontrivial stable equilibrium p-state $\hat{\mathcal{N}}(\mathcal{X})=\left(\hat{N}^{\mathbf{1}}(\mathcal{X}), \ldots, \hat{N}^{m}(\mathcal{X})\right)$ and we denote the feedback environment as it arises from an equilibrium p-state by $\hat{\boldsymbol{E}}(\mathcal{X})$.

\subsection{Invasion fitness and fitness proxies}

Our analysis is based on the mutation-limitation-based approximation of the evolutionary process in large populations known as Adaptive Dynamics (Metz et al. 1992, 1996a; Dieckmann and Law 1996; Geritz et al. 1998; Abrams 2005). Mutations are assumed to occur rarely so that the resident community is always at its equilibrium p-state at the moment a mutant enters the stage. We therefore distinguish two time scales. On the fast ecological time scale we follow the population dynamics after a mutation event until the new equilibrium p-state is reached. On the slow evolutionary time scale we follow the changing set of phenotypes $\mathcal{X}$ and the corresponding change in the equilibrium p-state $\hat{\mathcal{N}}(\mathcal{X})$ as a result of mutation and substitution events.

From the preceding paragraph follows that, when we consider the initial fate of a rare mutant, we do not have to deal with $\mathcal{N}_{t}$ but only with $\hat{\mathcal{N}}(\mathcal{X})$. Thus, the feedback environment in which a rare mutant has to prove itself is purely determined by $\mathcal{X}$ and we can write $\hat{\boldsymbol{E}}(\mathcal{X})$ instead of $\boldsymbol{E}\left(\mathcal{N}_{t}, \mathcal{X}\right)$.

Notation In order to avoid clutter we below customarily suppress the argument of $\hat{\boldsymbol{E}}$ and write $\hat{\boldsymbol{E}}$ for $\hat{\boldsymbol{E}}(\mathcal{X})$.

The population dynamics of a rare mutant is then described by Equation (1) with the matrix A composed of four types of entries: (i) unregulated and non-evolving demographic parameters $z_{l k}$, (ii) regulated but non-evolving demographic parameters $z_{l k} R_{\mathrm{z}, l k}(\hat{\boldsymbol{E}})$, (iii) unregulated traits $z_{l k}^{\prime}$, and (iv) regulated traits $z_{l k}^{\prime} R_{\mathrm{z}, l k}(\hat{\boldsymbol{E}})$.

In sufficiently large populations the fate of a rare mutant $\boldsymbol{x}^{\prime}$ is determined by its invasion fitness $\rho$, that is, its long term average growth rate in the environment determined by the resident community (Metz et al. 1992; Metz 2008). If $\rho>0$ the mutant has a positive probability to invade and increase in frequency. If $\rho<0$ the mutant is 


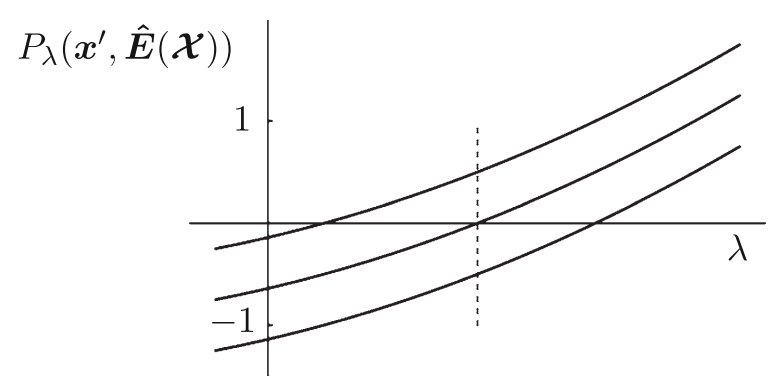

Fig. 3 Characteristic polynomial $P_{\lambda}\left(\boldsymbol{x}^{\prime}, \hat{\boldsymbol{E}}(\boldsymbol{\mathcal { X }})\right)$ for three different phenotypes $\boldsymbol{x}^{\prime}$ in the neighborhood of $\lambda=1$ (indicated by the vertical dotted line). The middle curve corresponds to a phenotype $\boldsymbol{x}^{\prime}$ that is part of the resident community $\mathcal{X}$. At equilibrium $P_{1}(\boldsymbol{x}, \hat{\boldsymbol{E}}(\boldsymbol{\mathcal { X }}))=0$. The other two curves correspond to mutant phenotypes $\boldsymbol{x}^{\prime}$ that are not part of the resident community. For the upper curve $P_{\lambda}\left(\boldsymbol{x}^{\prime}, \hat{\boldsymbol{E}}(\boldsymbol{\mathcal { X }})\right)=0$ is fulfilled for a $\lambda<1$. This is equivalent to $P_{1}\left(\boldsymbol{x}^{\prime}, \hat{\boldsymbol{E}}(\boldsymbol{\mathcal { X }})\right)>0$. For the lower curve the last two inequalities are reversed

doomed to extinction. For our model family, invasion fitness is given by the logarithm of the dominant eigenvalue $\lambda_{\mathrm{d}}$ of the matrix A. Generally $\rho=\ln \lambda_{\mathrm{d}}$ is a complicated mathematical expression. For analytical work it is therefore of interest to find algebraically simpler functions that provide at least qualitatively the same information. We call a fitness proxy any function $p$ for which $\operatorname{sign}[p]=\operatorname{sign}[\rho]$ and in the following we will introduce one such fitness proxy that is fundamental for our work. Let

$$
P_{\lambda}\left(\boldsymbol{x}^{\prime}, \hat{\boldsymbol{E}}\right):=\operatorname{det}(\lambda \mathrm{I}-\mathrm{A})
$$

where I denotes the identity matrix, be the characteristic polynomial of the projection matrix $A$. Since the resident community is assumed to be at its population dynamical attractor we have $\lambda_{\mathrm{d}}(\mathrm{A})=1$ for all $\boldsymbol{x}^{\prime}$ that are equal to a coordinate of $\mathcal{X}$. Furthermore, since $P_{\lambda}$ is a polynomial with leading coefficient +1 we have for $\boldsymbol{x}$ in $\mathcal{X}: P_{1}(\boldsymbol{x}, \hat{\boldsymbol{E}})=0$ and $\partial P_{1}(\boldsymbol{x}, \hat{\boldsymbol{E}}) / \partial \lambda>0$. Therefore, for mutant trait vectors $\boldsymbol{x}^{\prime}$ sufficiently similar to one of the resident trait vectors $\boldsymbol{x}$ in $\mathcal{X}$, we have $\lambda_{\mathrm{d}}\left(\boldsymbol{x}^{\prime}, \hat{\boldsymbol{E}}\right)>1 \Leftrightarrow P_{1}\left(\boldsymbol{x}^{\prime}, \hat{\boldsymbol{E}}\right)<0$ (Fig. 3). We define

$$
Q\left(\boldsymbol{x}^{\prime}, \hat{\boldsymbol{E}}\right):=-P_{1}\left(\boldsymbol{x}^{\prime}, \hat{\boldsymbol{E}}\right)
$$

Then, for $\boldsymbol{x}^{\prime}$ sufficiently close to $\boldsymbol{x} \in \mathcal{X}$,

$$
\operatorname{sign}\left[Q\left(\boldsymbol{x}^{\prime}, \hat{\boldsymbol{E}}\right)\right]=\operatorname{sign}\left[\ln \lambda_{\mathrm{d}}\left(\boldsymbol{x}^{\prime}, \hat{\boldsymbol{E}}\right)\right]
$$

Thus, $Q$ qualifies as a local fitness proxy. Metz and Leimar (2011) show that $Q$ can even be more powerful. More specifically, they show that (i) $Q\left(\boldsymbol{x}^{\prime}, \hat{\boldsymbol{E}}\right)>0$ implies $\ln \lambda_{\mathrm{d}}\left(\boldsymbol{x}^{\prime}, \hat{\boldsymbol{E}}\right)>0$ for any mutant trait vector $\boldsymbol{x}^{\prime}$ and (ii) if the trait space is path connected and $Q\left(\boldsymbol{x}^{\prime}, \hat{\boldsymbol{E}}\right)<0$ for all $\boldsymbol{x}^{\prime}$ that are not a coordinate of $\mathcal{X}$, then the community $\mathcal{X}$ is globally uninvadable.

Before we present an explicit biologically interpretable expression for $Q$ we have to introduce some terminology with respect to life cycles. We refer to Fig. 2 for an example of a life cycle graph that illustrates our terminology. 
Notation A loop is a sequence of demographic parameters $s_{l k}, f_{l k}$ that lead from one i-state to itself without passing through any i-state more than once. The loop transmission $L$ equals the product of the demographic parameters along the loop. In the remainder of this article we will use the terms loop and loop transmission synonymously and often denote a loop with its transmission. We denote the set of all i-states that are passed by loop $L$ by $L$. Two loops $L, M$ are connected if they share at least one i-state, i.e., if $\breve{L} \cap \breve{M} \neq \varnothing$, and unconnected otherwise. The set of all loops within a specific life cycle that is defined by the population projection matrix $A$ is denoted by $\mathcal{L}_{\mathrm{A}}$. A loop is called evolving when it contains at least one trait and regulated when it contains at least one regulated demographic parameter.

In Appendix A we show that $Q$ can be written as

$$
Q=-1+\sum_{\mathcal{L}_{\mathrm{A}}} L-\sum_{\mathcal{L}_{\mathrm{A}}^{2 *}} L M+\sum_{\mathcal{L}_{\mathrm{A}}^{3 *}} L M N-\ldots
$$

where $\mathcal{L}_{\mathrm{A}}^{k}$ denotes the $k$-fold Cartesian product over the set of loops $\mathcal{L}_{\mathrm{A}}$. The star indicates that $k$-tuples in which not all loops are unconnected to each other are excluded from the Cartesian product. Thus, the sums in Eq. (5) run over pairs, triplets, etc. of loops that are pairwise unconnected. Note that any number of loops can be a function of the mutant's trait vector $\boldsymbol{x}^{\prime}$ or can be affected by population regulation so that they are a function of the vector $\hat{\boldsymbol{E}}(\mathcal{\mathcal { X }})$. If $\mathrm{A}$ is a Leslie matrix describing an age-structured life cycle, then Eq. (5) simplifies to $Q=-1+\sum_{k=1}^{n} L_{k}$, where $L_{k}=f_{1 k} s_{k(k-1)} \ldots s_{21}$.

We conclude this subsection with some additional notation and a lemma.

Notation Let $\mathrm{A}$ be an $n \times n$ population projection matrix. For an index set $\alpha \subset$ $\{1, \ldots, n\}$ we denote the principal sub-matrix with indices in $\alpha$ by $\mathrm{A}_{\alpha}$. The index set obtained by eliminating the elements of $\alpha$ from $\{1, \ldots, n\}$ is denoted by $\backslash \alpha$. Thus, $\mathrm{A}_{\backslash \breve{L}}$ denotes the principal sub-matrix one obtains by deleting all rows and columns that correspond to i-states in the loop $L$.

By deleting a subset of i-states from a life cycle we obtain a reduced life cycle. While we denoted the fitness proxy based on the matrix A by $Q$, we denote the analogous fitness proxy but based on the matrix for a reduced life cycle, $\mathrm{A}_{\backslash \alpha}$, by $q(\alpha)$ :

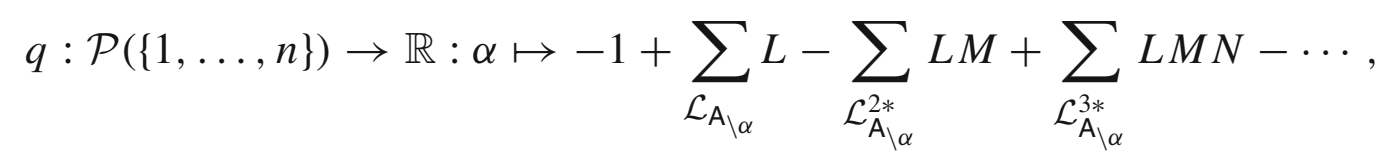

with $\mathcal{P}(\{1, \ldots, n\})$ denoting the power set of $\{1, \ldots, n\}$. Occasionally we will make use of the following lemma.

Lemma 1 (reduced life cycle) Let $\mathrm{A}$ be an $n \times n$ population projection matrix corresponding to a resident type at equilibrium and $\alpha \subset\{1, \ldots, n\}$. Then

$$
q(\alpha)<0
$$


Proof At equilibrium the dominant eigenvalue of the population projection matrix $A$ equals one: $\lambda_{d}(A)=1$. The population growth rate of an organism with a reduced life cycle is given by the logarithm of the dominant eigenvalue of the matrix $A$ where demographic parameters with indices in $\alpha$ are replaced with zeros. Let us denote this matrix by $\bar{A}$ with characteristic polynomial $\bar{P}$. The dominant eigenvalue of a non-negative matrix is strictly increasing in all matrix entries $a_{l k}$ (Horn and Johnson 1985). Thus, the dominant eigenvalue of the matrix $\overline{\mathrm{A}}$ has to be less than one. Note that $q(\alpha)$ is nothing else but the fitness proxy $Q$ calculated from $\overline{\mathrm{A}}: q(\alpha)=-\bar{P}_{1}\left(\boldsymbol{x}^{\prime}, \hat{\boldsymbol{E}}\right)$. The Lemma follows by using that $\lambda_{\mathrm{d}}(\overline{\mathrm{A}})<1 \Rightarrow-\bar{P}_{1}\left(\boldsymbol{x}^{\prime}, \hat{\boldsymbol{E}}\right)<0$, where the implication follows from the fact that $-\bar{P}_{1}\left(\boldsymbol{x}^{\prime}, \hat{\boldsymbol{E}}\right)$ decreases at the dominant eigenvalue and does not have any larger real roots than $\lambda_{d}(\bar{A})$.

Thus, if a resident type is at its population dynamical equilibrium, then removing i-states from the life cycle and replacing transitions to these states with death results in negative population growth. Under certain conditions Lemma 1 extends to mutant types $\boldsymbol{x}^{\prime}$.

Corollary 2 Let A be an $n \times n$ population projection matrix corresponding to a rare mutant type invading a resident type at equilibrium and $\alpha \subset\{1, \ldots, n\}$. Then $q(\alpha)<0$ (i) for all mutant types sufficiently similar to the resident type, (ii) for all mutant types if all loops $L \in \mathcal{L}_{\mathrm{A}_{\downarrow \alpha}}$ are not evolving or (iii) for all mutant types if all loops $L \in \mathcal{L}_{\mathrm{A}_{\backslash \alpha}}$ are not regulated.

Proof (i) This result follows by continuity. (ii) If loops $L \in \mathcal{L}_{\mathrm{A}_{\backslash \alpha}}$ are not evolving then $q(\alpha)$ does not depend on $\boldsymbol{x}^{\prime}$ and hence $q(\alpha)<0$ for all $\boldsymbol{x}^{\prime}$. (iii) Assume loops $L \in \mathcal{L}_{\mathrm{A}_{\backslash \alpha}}$ are not regulated and $q(\alpha)>0$ for a mutant $\boldsymbol{x}^{\prime}$. Then the type $\boldsymbol{x}^{\prime}$ would as resident grow unboundedly on the reduced life cycle alone. This is in contradiction to the assumption that the model state converges to an equilibrium for all $\boldsymbol{x}$. Therefore, $q(\alpha)<0$ for all $\boldsymbol{x}^{\prime}$.

\subsection{Singular points and evolutionary dynamics}

Invasion fitness is a fundamental tool in predicting long-term evolutionary dynamics. If mutations have small phenotypic effect, a mutant which invades will go to fixation if it cannot be invaded by the former resident when common itself (Dercole et al. 2002; Geritz et al. 2002; Geritz 2005; Dercole and Rinaldi 2008). The evolutionary dynamics can then be determined by following a series of mutation-substitution events (Dieckmann and Law 1996; Metz et al. 1996a; Geritz et al. 1998).

Here we will be interested in points $\boldsymbol{x}^{*}$ in trait space where, given a single resident type, the fitness landscape has a stationary point, i.e.,

$$
0=\left.\frac{\partial \rho\left(\boldsymbol{x}^{\prime}, \hat{\boldsymbol{E}}\left(\boldsymbol{x}^{*}\right)\right)}{\partial x_{j}^{\prime}}\right|_{\boldsymbol{x}^{\prime}=\boldsymbol{x}^{*}} \text { for all coordinates } x_{j} \text { of } \boldsymbol{x}^{\prime}
$$

or, equivalently, where such an equality holds for a fitness proxy $p$. Such points are equilibria of monomorphic adaptive dynamics and are called candidate ESSs (e.g. 
Ellner and Hairston 1994), potential ESSs (e.g. Otto and Day 2007) or evolutionarily singular points (Metz et al. 1996a; Geritz et al. 1998). Two properties of singular points are of particular importance: invadability and attractivity (traditionally called convergence stability). A singular point is uninvadable when it is at a maximum of the fitness landscape and invadable when it is not. A singular point in a one-dimensional trait space attracts when a neighbourhood of the point exists such that a mutant that deviates but slightly from a resident type within that neighbourhood can only invade if it is more similar to the singular point. Attracting singular points are attractors of monomorphic adaptive dynamics. Singular points that are not attracting are evolutionarily repelling and a population evolves away from them. In multi-dimensional trait spaces attractivity is a more complex concept, i.a., because the evolutionary dynamics is also governed by the covariation between the effects a mutation induces in the different traits (Leimar 2009).

In one-dimensional trait spaces singular points can be classified according to these two properties in the following way (Metz et al. 1996a; Geritz et al. 1998). Singular points that are both attracting and uninvadable are final stops of evolution. Singular points that are attracting but invadable by nearby mutants are called evolutionary branching points (Metz et al. 1996a; Geritz et al. 1998). Selection initially acts in the direction of these points, but once the resident trait value is sufficiently close to the singular point selection turns disruptive and favors an increase in phenotypic variance (Rueffler et al. 2006). In the case of clonal organisms this increase can be realised by the emergence of two independent lineages and it is this scenario that earned such points their name. Invadable singular points that lack attractivity are evolutionary repellors while uninvadable and nonattracting singular points are known as Garden of Eden-points (Nowak 1990). Both are repellors of the evolutionary dynamics and populations evolve away from these points.

\subsection{Optimisation, the feedback environment and frequency dependence}

In life history theory predictions were traditionally not derived from an invasion analysis but based on the maximization of quantities like the basic reproduction ratio $R_{0}$ or the intrinsic growth rate $r$ (e.g. Stearns 1992; Charnov 1993; Charlesworth 1994; Roff 2002). However, in a density-dependent setting this idea becomes ambiguous because at demographic equilibrium necessarily $R_{0}=1$ and $r=0$. Recently there is an increased awareness that an optimisation principle has to be derived from an invasion argument (Metz et al. 1992; Mylius and Diekmann 1995; Pásztor et al. 1996; Dieckmann and Metz 2006). Metz et al. (1996b, 2008a) and Gyllenberg and Service (2011) derived necessary and sufficient conditions for optimisation to be possible. The following definitions and propositions come almost straight from the articles by Metz et al. and we refer to them for proofs. The next definitions introduce some of our language use.

Definition 3 The trait vector acts in a monotone and one-dimensional manner whenever there exists a function $\psi: \mathscr{X} \rightarrow \mathbb{R}$ and a function $\alpha: \mathbb{R} \times \mathscr{E} \rightarrow \mathbb{R}$ which increases in its first argument such that 


$$
\operatorname{sign}\left[\ln \lambda_{\mathrm{d}}\left(\boldsymbol{x}^{\prime}, \hat{\boldsymbol{E}}\right)\right]=\operatorname{sign}\left[\alpha\left(\psi\left(\boldsymbol{x}^{\prime}\right), \hat{\boldsymbol{E}}\right)\right]
$$

Definition 4 The feedback-environment acts in a monotone and one-dimensional manner whenever there exists a function $\phi: \mathscr{E} \rightarrow \mathbb{R}$ and a function $\beta: \mathscr{X} \times \mathbb{R} \rightarrow \mathbb{R}$ which increases in its second argument such that

$$
\operatorname{sign}\left[\ln \lambda_{\mathrm{d}}\left(\boldsymbol{x}^{\prime}, \hat{\boldsymbol{E}}\right)\right]=\operatorname{sign}\left[\beta\left(\boldsymbol{x}^{\prime}, \phi(\hat{\boldsymbol{E}})\right)\right]
$$

Definition 5 Given an eco-evolutionary model, an optimisation principle is a function $\psi: \mathscr{X} \rightarrow \mathbb{R}$ such that for any constraint on $\mathscr{X}$ the attracting and uninvadable singular points for this model can be calculated by maximising $\psi$.

Definition 6 Given an eco-evolutionary model, a pessimization principle is a function $\phi: \mathscr{E} \rightarrow \mathbb{R}$ such that for any constraint on $\mathscr{E}$ the attracting and uninvadable singular points for this model can be calculated by minimizing $\phi$.

Proposition 7 Models have an optimisation principle if and only if the trait vector acts in a monotone and one-dimensional manner.

Proposition 8 Models have a pessimization principle if and only if the environment acts in a monotone and one-dimensional manner.

Proposition 9 Models that have an optimisation principle $\psi$ also have a pessimization principle $\phi$ and vice versa (just take $\psi(\boldsymbol{x})=-\phi(\hat{\boldsymbol{E}}(\boldsymbol{x}))$.

In Sect. 3 we state necessary and sufficient conditions for the existence of optimisation principles and in Sect. 3.2 we give concrete methods to find optimisation principles for our model family. If an optimisation principle exists, then the outcome of any resident-mutant dynamics is easily predicted: The type corresponding to the higher value of the optimisation principle eventually displaces the type corresponding to the lower value of the optimisation principle. The evolutionary dynamics in the presence of an optimisation principle is of a rather simple kind because then attractivity and uninvadability are no longer independent properties: singular points attract if and only if they are uninvadable (e.g. Metz et al. 1996b, 2008a; Rueffler et al. 2004). In particular, with an optimisation principle protected polymorphisms caused by a rare-type advantage are impossible. In conclusion, knowing that an optimisation principle exists tells that the evolutionary dynamics is of the simplest possible kind. Moreover, optimisation principles in the form of simple explicit formulas foster the quick calculation of uninvadable singular points.

Finding an optimisation principle that serves this purpose can fail for two reasons. First, it may fail because no general algorithm exists that delivers practical optimisation principles in all circumstances where optimisation is possible. (The algorithm presented in Gyllenberg and Service (2011) is of mathematical interest but not practical. The reason is that for its calculation infinitely many evaluations of $\rho\left(\boldsymbol{x}^{\prime}, \hat{\boldsymbol{E}}\right)$ have to be performed. In contrast, the optimization principles put forward in this article are all algebraic functions.) Second, it has to fail whenever an optimisation principle does not exist for structural reasons. This is the case when population regulation acts in a way 
such that different individuals have a different influence on and a different perception of the environment and we refer to selection under such conditions as frequencydependent. Selection is frequency-dependent if and only if no optimization principle exists. Two cases can be distinguished. First, an optimization principle does not exist when the trait vector or the feedback environment acts in a one-dimensional manner but not monotonically so. It is then possible that three strategies exists that can invade each other according to a rock-paper-scissors game (Gyllenberg and Service 2011). Second, an optimization principle does not exist if the trait vector or the feedback environment does not act in a one-dimensional manner (Heino et al. 1998; Diekmann 2004). A necessary prerequisite for this is that population regulation occurs through more than one feedback variable. Note, that our definition of frequency dependence is geared towards long-term evolution driven by mutation and substitution events and therefore differs from the classical definition of population genetics which deals with the change of allele frequencies (Heino et al. 1998). For one-dimensional trait spaces it can be tested numerically whether selection is frequency-dependent by looking at sign plots of invasion fitness $\lambda_{\mathrm{d}}\left(\boldsymbol{x}^{\prime}, \hat{\boldsymbol{E}}\right)$ (so-called pairwise invadabilitly plots or PIPs (Metz et al. 1996a; Geritz et al. 1998)). One such procedure is described in Gyllenberg and Service (2011) and another one, allowing exhaustive visual checking, in Metz et al. (2008a).

It is under frequency dependence that attractivity and invadability become mutually independent properties. Then, comparing the curvature of the contour lines of the fitness landscape and the trade-off curve at a singular point generally only allows determining whether a singular point is uninvadable but not whether it attracts. However, in Sect. 6 we show that for models where so-called invasion boundaries are linear it is possible to go one step further.

\subsection{Invasion boundaries}

In the case of two-dimensional trait spaces a fitness landscape as generated by a given resident community can be visualized as a three-dimensional plot. In the context of an invasion analysis it is sufficient to know whether a rare mutant type has a positive or negative invasion fitness. Therefore it is most useful to visualize a fitness landscape as a contour plot with a single contour at height zero. The contour line is given by all pairs $\left(x_{1}, x_{2}\right)$ that are selectively neutral with respect to a given resident community $\mathcal{X}$. We express this set by the condition $x_{2}=I\left(x_{1}\right)$ with $I\left(x_{1}\right)$ implicitly defined by

$$
\rho\left(\left(x_{1}, I\left(x_{1}\right)\right), \hat{\boldsymbol{E}}\right)=0
$$

In this equation we may replace $\rho$ with any fitness proxy $p$ since by definition all zeros of $\rho$ are also zeros of $p$. Since fitness is an increasing function of all demographic parameters, the contour lines of the fitness landscape have negative slope: $\mathrm{d} I / \mathrm{d} x_{1}<0$. We refer to $I\left(x_{1}\right)$ as invasion boundary because for each resident community it divides the positive cone $\mathbb{R}_{+}^{2}$ into an inward part, adjacent to the origin, and an outward part. Trait vectors $\left(x_{1}, x_{2}\right)$ that lie in the outward part are able to invade since for them $p\left(\left(x_{1}^{\prime}, x_{2}^{\prime}\right), \hat{\boldsymbol{E}}\right)>0$ while trait vectors that lie in the outward part 
are not able to invade since for them $p\left(\left(x_{1}^{\prime}, x_{2}^{\prime}\right), \hat{\boldsymbol{E}}\right)<0$. In Fig. 1 the contour lines that pass through the dots on the trade-off curves represent invasion boundaries given that the dot indicates the strategy of the resident type. Since at population dynamical equilibrium $p\left(\boldsymbol{x}^{j}, \hat{\boldsymbol{E}}\right)=0$ for all resident trait vectors $\boldsymbol{x}^{j}$ in $\mathcal{X}$, invasion boundaries either intersect with or are tangent to the trade-off curve $\left\{\left(x_{1}, x_{2}\right) \mid x_{2}=T\left(x_{1}\right)\right\}$ at $\boldsymbol{x}^{j}$. The fact that at singular points $\boldsymbol{x}^{*}$ the fitness gradient is zero implies that invasion boundaries are tangent to the trade-off curve at $\boldsymbol{x}^{*}$ (Rueffler et al. 2004). A singular point is a fitness maximum and therefore uninvadable if the invasion boundary, except for the singular point, lies, at least locally, outside the feasibility set delineated by the trade-off curve. This is true if and only if at the singular point $\mathrm{d} T^{2} / \mathrm{d} x_{1}^{2}<\mathrm{d} I^{2} / \mathrm{d} x_{1}^{2}$. Conversely, a singular point is a fitness minimum and therefore invadable if the invasion boundary, except for the singular point, lies, at least locally, inside the feasibility set, corresponding to $\mathrm{d} T^{2} / \mathrm{d} x_{1}^{2}>\mathrm{d} I^{2} / \mathrm{d} x_{1}^{2}$. Thus, depending on the curvature of the invasion boundary at a singular point, singular points are invadable for either a wide or a narrow range of possible trade-off curves, and uninvadable for the complement (Rueffler et al. 2004; de Mazancourt and Dieckmann 2004; Bowers et al. 2005). If an optimisation principle is known, for our class of models the evolutionary outcome can be determined by identifying the local extrema of the optimisation principle and then comparing the curvature of the invasion boundary at the extrema with the curvature of the trade-off.

In Sect. 5 we derive for the case of two traits simple conditions that determine whether invasion boundaries are convex $\left(\mathrm{d}^{2} I / \mathrm{d} x_{2}^{2}>0\right)$, linear $\left(\mathrm{d}^{2} I / \mathrm{d} x_{2}^{2}=0\right)$ or concave $\left(\mathrm{d}^{2} I / \mathrm{d} x_{2}^{2}<0\right)$ and in Sect. 5.2 we show that for models with a high degree of symmetry these results can be extended to models with an arbitrary number of traits.

To be able to compare the curvature of invasion boundaries and trade-offs we will use the following conventions. If both curves are convex at a singular point, i.e., have a positive second derivative, we will say that one curve is more strongly convex than the other if the former has a larger value of its second derivative than the latter and we will say that one curve is less strongly convex than the other if the former has a smaller value of its second derivative than the latter. If both curves are concave at the singular point, hence, have a negative second derivative, we use the expressions more strongly concave and less strongly concave. These are defined as above but now the absolute value of the second derivatives has to be compared. In other words, if the trade-off is concave, a singular point is locally uninvadable if the invasion boundary is less strongly concave, linear or convex. If the trade-off is convex, a singular point is locally uninvadable if the invasion boundary is more strongly convex. In conclusion, the more strongly convex invasion boundaries are, the wider is the range of trade-off curvatures that result in uninvadable singular points.

\subsection{Limitations of the approach}

In the previous subsections we assumed, as is usual in discussions based on the adaptive dynamics approximation, clonal reproduction and rare as well as small mutational steps. In this subsection we give a brief, necessarily heuristic, overview of how far we expect our results to reach. 
Under relatively mild conditions most of our results also apply to sexually reproducing diploids (Van Dooren 2006, in press; Metz 2008). It suffices that (i) invasion success of mutants can be considered for each mutation separately, and (ii) mutant heterozygotes are roughly on the line segment between the resident and the mutant homozygote trait values. Although there are no good arguments why genetics should be even approximately additive on a large scale, a case can be made that most relevant mutations have small effect (Metz 2005, 2011) and that for such mutations this effect is close to additive (Van Dooren, in press), while moreover the change in the environment due to the gene substitution can to first order be neglected (Meszéna et al. 2005). Together this implies both (i) and (ii).

Close to singular points, or with larger deviations from additive genetics, protected polymorphisms appear more often in diploid than in clonal models (Schneider 2006; Van Dooren 2006). However, in cases where a clonal model predicts evolution towards a singular point, those polymorphisms are often transient, in that they are taken over by single mutants that go to fixation (Van Dooren 2006). If the latter occurs, clonal models once again correctly predict the outcome of the long-term evolutionary dynamics also for the sexual diploid case.

Generally, $Q$ is a valid fitness proxy only if the mutant type is sufficiently similar to the resident. In Appendix A we use the approach of Metz and Leimar (2011) to show that if the trait space is path connected and a candidate optimisation principle $\psi$ can be derived from $Q$, then, generically, $\operatorname{sign}\left[Q\left(\boldsymbol{x}^{\prime}, \hat{\boldsymbol{E}}\right)\right]=\operatorname{sign}\left[\ln \lambda_{\mathrm{d}}\left(\boldsymbol{x}^{\prime}, \hat{\boldsymbol{E}}\right)\right]$ for any mutant trait vector $\boldsymbol{x}^{\prime}$, implying that $\psi$ is indeed a full optimisation principle.

\section{Optimisation}

From this section on we present our results. Here we list conditions under which evolutionary predictions can be derived from an optimisation principle. These results are general in the sense that they hold for models with an arbitrary number of traits and regulated parameters. This section consists of three subsections. In the first one we present sufficient conditions that allow to derive optimisation principles based on the fitness proxy $Q$. In the next subsection we present an extensive catalogue of optimisation principles based on these conditions. In the final subsection we collect various optimisation principles that are not based on $Q$.

\subsection{Optimisation based on $Q$}

Sufficient conditions such that an optimisation principle based on the fitness proxy $Q$ can be derived.

Proposition 10 Assume that functions $g_{i}: \mathscr{X} \rightarrow \mathbb{R}$ and $e_{i}: \mathscr{E} \rightarrow \mathbb{R}$ for $i \in\{1,2\}$ with $g_{2} \neq 0$ exist such that

$$
Q\left(\boldsymbol{x}^{\prime}, \hat{\boldsymbol{E}}\right)=g_{1}\left(\boldsymbol{x}^{\prime}\right) e_{1}(\hat{\boldsymbol{E}})+g_{2}\left(\boldsymbol{x}^{\prime}\right) e_{2}(\hat{\boldsymbol{E}})
$$

If $e_{1}>0$, then $g_{1} /\left|g_{2}\right|$ is an optimisation principle. If $e_{1}<0$, then $-g_{1} /\left|g_{2}\right|$ is an optimisation principle. 
Proof Dividing Eq. (11) by $\left|g_{2}\left(\boldsymbol{x}^{\prime}\right)\right|$ results in

$$
\begin{aligned}
\frac{Q\left(\boldsymbol{x}^{\prime}, \hat{\boldsymbol{E}}\right)}{\left|g_{2}\left(\boldsymbol{x}^{\prime}\right)\right|} & =\frac{g_{1}\left(\boldsymbol{x}^{\prime}\right)}{\left|g_{2}\left(\boldsymbol{x}^{\prime}\right)\right|} e_{1}(\hat{\boldsymbol{E}})+\frac{g_{2}\left(\boldsymbol{x}^{\prime}\right)}{\left|g_{2}\left(\boldsymbol{x}^{\prime}\right)\right|} e_{2}(\hat{\boldsymbol{E}}) \\
& =\frac{g_{1}\left(\boldsymbol{x}^{\prime}\right)}{\left|g_{2}\left(\boldsymbol{x}^{\prime}\right)\right|} e_{1}(\hat{\boldsymbol{E}})+\operatorname{sign}\left[g_{2}\left(\boldsymbol{x}^{\prime}\right)\right] e_{2}(\hat{\boldsymbol{E}})
\end{aligned}
$$

Then $\operatorname{sign}\left[Q\left(\boldsymbol{x}^{\prime}, \hat{\boldsymbol{E}}\right) /\left|g_{2}\left(\boldsymbol{x}^{\prime}\right)\right|\right]=\operatorname{sign}\left[Q\left(\boldsymbol{x}^{\prime}, \hat{\boldsymbol{E}}\right)\right]$ and therefore $Q\left(\boldsymbol{x}^{\prime}, \hat{\boldsymbol{E}}\right) /\left|g_{2}\left(\boldsymbol{x}^{\prime}\right)\right|$ is a fitness proxy. Choose $\psi(\boldsymbol{x})=g_{1}(\boldsymbol{x}) /\left|g_{2}(\boldsymbol{x})\right|$ if $e_{1}>0$, or $\psi(\boldsymbol{x})=-g_{1}(\boldsymbol{x}) /\left|g_{2}(\boldsymbol{x})\right|$ if $e_{1}<0$, and $\alpha(\psi(\boldsymbol{x}), \hat{\boldsymbol{E}})=\psi(\boldsymbol{x}) e_{1}(\hat{\boldsymbol{E}})+\operatorname{sign}\left[g_{2}\left(\boldsymbol{x}^{\prime}\right)\right] e_{2}(\hat{\boldsymbol{E}})$. The result then follows from Proposition 7.

Corollary 11 (i) Assume that functions $g_{1}: \mathscr{X} \rightarrow \mathbb{R}, e_{1}: \mathscr{E} \rightarrow \mathbb{R}$ and $e_{2}: \mathscr{E} \rightarrow$ $\mathbb{R}$ exist such that $Q\left(\boldsymbol{x}^{\prime}, \hat{\boldsymbol{E}}\right)=g_{1}\left(\boldsymbol{x}^{\prime}\right) e_{1}(\hat{\boldsymbol{E}})+e_{2}(\hat{\boldsymbol{E}})$. If $e_{1}>0$, then $g_{1}$ is an optimisation principle. If $e_{1}<0$, then $-g_{1}$ is an optimisation principle.

(ii) Assume that functions $g_{1}: \mathscr{X} \rightarrow \mathbb{R}, g_{2}: \mathscr{X} \rightarrow \mathbb{R}$ and $e_{1}: \mathscr{E} \rightarrow \mathbb{R}$ exist such that $Q\left(\boldsymbol{x}^{\prime}, \hat{\boldsymbol{E}}\right)=g_{1}\left(\boldsymbol{x}^{\prime}\right) e_{1}(\hat{\boldsymbol{E}})+g_{2}\left(\boldsymbol{x}^{\prime}\right)$. If $g_{1} \neq 0$, then $g_{2} /\left|g_{1}\right|$ is an optimisation principle. Alternatively, and provided $g_{2} \neq 0$ the following optimisation principles exist. If $e_{1}>0$, then $g_{1} /\left|g_{2}\right|$ is an optimisation principle and if $e_{1}<0$, then $-g_{1} /\left|g_{2}\right|$ is an optimisation principle.

An even more special case is at hand when traits and regulatory functions do not co-occur in a product.

Corollary 12 Assume that functions $g: \mathscr{X} \rightarrow \mathbb{R}$ and $e: \mathscr{E} \rightarrow \mathbb{R}$ exist such that $Q\left(\boldsymbol{x}^{\prime}, \hat{\boldsymbol{E}}\right)=g\left(\boldsymbol{x}^{\prime}\right)+e(\hat{\boldsymbol{E}})$. Then the function $g$ is an optimisation principle.

\subsection{Applications of Proposition 10}

In simple life cycles it is often easy to check whether the terms in $Q$ can be rearranged such that Proposition 10 is applicable. However, in more complicated ones this can be tedious. In this subsection we present a comprehensive list of conditions such that Proposition 10 is applicable. We leave it as a challenge to the reader either to come up with cases not covered by our list or to prove that this list is complete.

For each element in our list of optimisation principles we give an example. These examples are based on the life cycles shown in Fig. 4 which we describe here briefly. Fig. 4a shows the age-structured life cycle of an iteroparous plant species with seed bank. The fitness proxy $Q$ is given by

$$
Q=-1+\tilde{s}_{11}+\tilde{f}_{13} \tilde{s}_{32} \tilde{s}_{21}+\tilde{f}_{14} \tilde{s}_{43} \tilde{s}_{32} \tilde{s}_{21}
$$

where for convenience we dropped the arguments of $Q$. Fig. $4 \mathrm{~b}$ shows a size-structured life cycle with three size classes with only individuals in the largest one capable of reproduction. The fitness proxy $Q$ can be written as

$$
Q=\tilde{f}_{13} \tilde{s}_{32} \tilde{s}_{21}-\left(1-\tilde{s}_{11}\right)\left(1-\tilde{s}_{22}\right)\left(1-\tilde{s}_{33}\right) \text {. }
$$


(a)

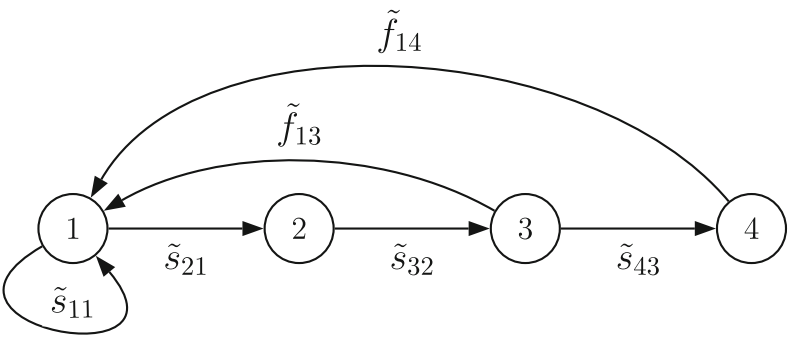

(b)

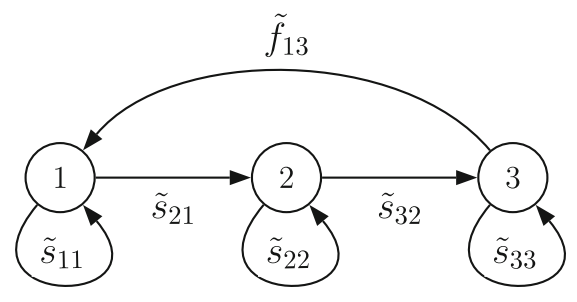

(c)

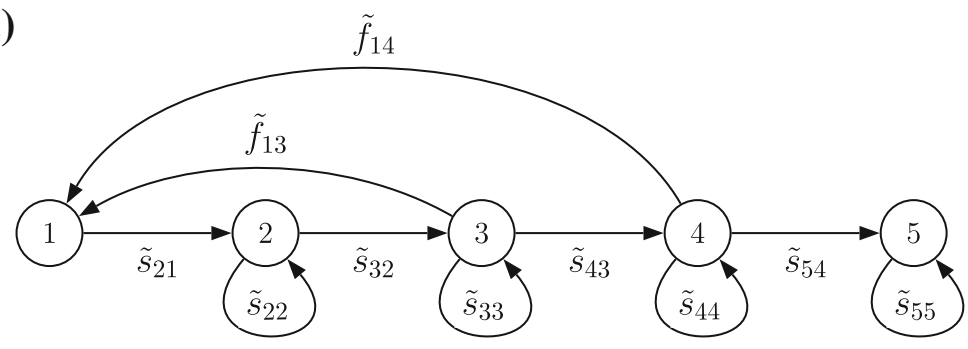

Fig. 4 Life cycle graphs that go with the examples in Sect. 3.2. The corresponding fitness proxies are given in Eq. 12

Figure 4c shows a stage structured life cycle with one post-reproductive class, similar to the life cycle suggested by Brault and Caswell (1993) for the killer whale Orcinus orca. The fitness proxy $Q$ can be written as

$$
\begin{aligned}
Q= & \tilde{f}_{13} \tilde{s}_{32} \tilde{s}_{21}\left(1-\tilde{s}_{44}\right)\left(1-\tilde{s}_{55}\right)+\tilde{f}_{14} \tilde{s}_{43} \tilde{s}_{32} \tilde{s}_{21}\left(1-\tilde{s}_{55}\right) \\
& -\left(1-\tilde{s}_{22}\right)\left(1-\tilde{s}_{33}\right)\left(1-\tilde{s}_{44}\right)\left(1-\tilde{s}_{55}\right) .
\end{aligned}
$$

For most of these examples the developed theory is an overkill. Yet, they may serve to help understanding the general strategy. More examples can be found in Sect. 7. Remember that we label demographic parameters with a tilde to indicate that they potentially contain a regulatory function. On the other hand, traits are never labelled with a tilde since we then only refer to the hereditary component of a demographic parameter.

Notation The formulation of the following results relies on book-keeping of loops and whether or not they contain traits or regulatory functions. Here we introduce notation facilitating this. We collect all traits and regulatory functions occurring in a given eco-evolutionary model in the vectors $\boldsymbol{x}$ and $\boldsymbol{R}$. For a given loop $L$, we collect the traits and regulatory functions present in that loop in the vectors $\boldsymbol{x}_{L}$ and $\boldsymbol{R}_{L}$. For a set of loops $\mathcal{M} \subseteq \mathcal{L}_{\mathrm{A}}$ we collect the traits and regulatory functions present in the loops $L \in \mathcal{M}$ in the vectors $\boldsymbol{x}_{\mathcal{M}}$ and $\boldsymbol{R}_{\mathcal{M}}$. We denote the set of all entries in a vector $\boldsymbol{x}$ or $\boldsymbol{R}$ by $\breve{\boldsymbol{x}}$ and $\breve{\boldsymbol{R}}$, respectively, e.g., if $\boldsymbol{x}=\left(f_{11}, f_{12}, f_{13}\right)$, then $\breve{\boldsymbol{x}}=\left\{f_{11}, f_{12}, f_{13}\right\}$. 
Furthermore, we define the following short-hand notation: $L / x_{L}:=L / \prod_{\breve{x}_{L}} x_{j}$ and $L / \boldsymbol{R}_{L}:=L / \prod_{\breve{R}_{L}} R_{j}$, where the subscript $j$ is a multi-index. If we wish to stress the dependence of a loop, a matrix or a reduced life cycle on traits or regulatory functions, we add $(\boldsymbol{x})$ and $(\boldsymbol{R})$, respectively, as arguments, for instance, $L(\boldsymbol{x}), \mathrm{A}(\boldsymbol{R})$ or $q(\alpha)(\boldsymbol{x})$. This notation does not mean that a loop or matrix necessarily contains all traits or regulatory functions but indicates that a loop or matrix may depend on traits or regulatory functions. If we divide a loop by the traits or regulatory functions present in it and want to make a particular dependence explicit we write for example $\left[L / \boldsymbol{x}_{L}\right](\boldsymbol{R})$. With some abuse of notation we use $\boldsymbol{R}$ instead of $\hat{\boldsymbol{E}}$ as the argument representing the resident type. Thus, we write $Q\left(\boldsymbol{x}^{\prime}, \boldsymbol{R}\right)$ instead of $Q\left(\boldsymbol{x}^{\prime}, \hat{\boldsymbol{E}}\right)$.

As first prerequisite for the application of Proposition 10 we split the terms in $Q$ in two groups, which we generically label as $a$ and $b$. Thus,

$$
Q\left(\boldsymbol{x}^{\prime}, \boldsymbol{R}\right)=a+b
$$

The fitness proxy $Q$ contains the term -1 and, without restriction of generality, we assume it is part of $b$. Grouping the terms in $Q$ into $a$ and $b$ can be done in two qualitatively different ways. In the first case, the sets of loops occurring in $a$ and $b$ are disjunct. This is possible if and only if a set of loops $\mathcal{M} \subset \mathcal{L}_{\mathrm{A}}$ exists such that all loops $L \in \mathcal{M}$ are connected to all loops $L \in \mathcal{L}_{\mathrm{A}} \backslash \mathcal{M}$. Then

$$
\begin{aligned}
Q= & \underbrace{\sum_{\mathcal{M}} L-\sum_{\mathcal{M}^{2 *}} L M+\sum_{\mathcal{M}^{3 *}} L M N-\cdots}_{a} \\
& +\underbrace{\left(-1+\sum_{\mathcal{L}_{\mathrm{A}} \backslash \mathcal{M}} L-\sum_{\left(\mathcal{L}_{\mathrm{A}} \backslash \mathcal{M}\right)^{2 *}} L M+\sum_{\left(\mathcal{L}_{\mathrm{A}} \backslash \mathcal{M}\right)^{3 *}} L M N-\cdots\right)}_{b} .
\end{aligned}
$$

In the second case the sets of loops in $a$ and $b$ are not disjunct but intersecting. Such a split can be done in several ways. For our purpose it is useful to consider the case that a set of loops $\mathcal{M} \subset \mathcal{L}_{\mathrm{A}}$ exists that is exclusive to $a$. Then

$$
\begin{aligned}
& Q=\sum_{\mathcal{M}} L\left(1-\sum_{\mathcal{L}_{\mathrm{A}_{\backslash \breve{L}}}} M+\sum_{\mathcal{L}_{\AA_{\backslash \breve{L}}^{2 *}}^{2 *}} M N-\cdots\right) \\
& -1+\sum_{\mathcal{L}_{\mathrm{A}} \backslash \mathcal{M}} L-\sum_{\left(\mathcal{L}_{\mathrm{A}} \backslash \mathcal{M}\right)^{2 *}} L M+\sum_{\left(\mathcal{L}_{\mathrm{A}} \backslash \mathcal{M}\right)^{3 *}} L M N-\cdots \\
& =\underbrace{-\sum_{\mathcal{M}} L q(\breve{L})}_{a}+\underbrace{\left(-1+\sum_{\mathcal{L}_{\mathrm{A}} \backslash \mathcal{M}} L-\sum_{\left(\mathcal{L}_{\mathrm{A}} \backslash \mathcal{M}\right)^{2 *}} L M+\sum_{\left(\mathcal{L}_{\mathrm{A}} \backslash \mathcal{M}\right)^{3 *}} L M N-\cdots\right)}_{b}
\end{aligned}
$$

where $q(\breve{L})$ is defined in Sect. 2.2.

In some cases the fitness proxy $Q$ and the basic reproduction ratio $R_{0}$ are related to each other in a very simple manner (Rueffler, submitted): if the life cycle contains only 
a single birth state and if $\mathcal{M}$ contains exactly those loops containing a fertility parameter $\tilde{f}_{1 j}$, then $R_{0}=-a / b$ with $a$ and $b$ as defined in Eq. (15) (Appendix A). Thus, some of the optimisation principles listed below can be interpreted as components of $R_{0}$.

For both $a$ and $b$ holds that they can either only depend on $\boldsymbol{x}$, only depend on $\boldsymbol{R}$ or depend on both $\boldsymbol{x}$ and $\boldsymbol{R}$. Thus, $Q$ can have one of the following structures:

$$
\begin{aligned}
& Q\left(\boldsymbol{x}^{\prime}, \boldsymbol{R}\right)=a\left(\boldsymbol{x}^{\prime}\right)+b(\boldsymbol{R}) \\
& Q\left(\boldsymbol{x}^{\prime}, \boldsymbol{R}\right)=a(\boldsymbol{R})+b\left(\boldsymbol{x}^{\prime}\right) \\
& Q\left(\boldsymbol{x}^{\prime}, \boldsymbol{R}\right)=a\left(\boldsymbol{x}^{\prime}\right)+b\left(\boldsymbol{x}^{\prime}, \boldsymbol{R}\right) \\
& Q\left(\boldsymbol{x}^{\prime}, \boldsymbol{R}\right)=a(\boldsymbol{R})+b\left(\boldsymbol{x}^{\prime}, \boldsymbol{R}\right) \\
& Q\left(\boldsymbol{x}^{\prime}, \boldsymbol{R}\right)=a\left(\boldsymbol{x}^{\prime}, \boldsymbol{R}\right)+b\left(\boldsymbol{x}^{\prime}\right) \\
& Q\left(\boldsymbol{x}^{\prime}, \boldsymbol{R}\right)=a\left(\boldsymbol{x}^{\prime}, \boldsymbol{R}\right)+b(\boldsymbol{R}) \\
& Q\left(\boldsymbol{x}^{\prime}, \boldsymbol{R}\right)=a\left(\boldsymbol{x}^{\prime}, \boldsymbol{R}\right)+b\left(\boldsymbol{x}^{\prime}, \boldsymbol{R}\right)
\end{aligned}
$$

If Eq. (14) or (15) can be written as in Eq. (16a) or (16b), then it follows from Corollary 12 that $a\left(\boldsymbol{x}^{\prime}\right)$ and $b\left(\boldsymbol{x}^{\prime}\right)$, respectively, are optimisation principles. If, however, $a$ or $b$ depends on both $\boldsymbol{x}$ and $\boldsymbol{R}$, then Proposition 10 can only be applied if it is possible to write $a\left(\boldsymbol{x}^{\prime}, \boldsymbol{R}\right)$ or $b\left(\boldsymbol{x}^{\prime}, \boldsymbol{R}\right)$ as a product of two factors, one only depending on $\boldsymbol{x}$ and the other only depending on $\boldsymbol{R}$. In the following we list conditions such that (i) $a$ and $b$ only depend on either traits or regulatory functions or (ii) $a$ and $b$ can be split into two factors, each of which only depends on either traits or regulatory functions.

In case of disjunct sets of loops, $Q\left(\boldsymbol{x}^{\prime}, \boldsymbol{R}\right)=a\left(\boldsymbol{x}^{\prime}\right)+b(\boldsymbol{R})$ or $Q\left(\boldsymbol{x}^{\prime}, \boldsymbol{R}\right)=a(\boldsymbol{R})+$ $b\left(\boldsymbol{x}^{\prime}\right)$ if loops $L \in \mathcal{M}$ depend only on either $\boldsymbol{x}$ or $\boldsymbol{R}$ while loops $L \in \mathcal{L}_{\mathrm{A}} \backslash \mathcal{M}$ only depend on either $\boldsymbol{R}$ or $\boldsymbol{x}$, respectively. In case of intersecting sets of loops an additional requirement has to hold true: Loops unconnected to loops $L \in \mathcal{M}$ contain neither traits nor regulatory functions. Then

$$
a\left(\boldsymbol{x}^{\prime}\right)=-\sum_{\mathcal{M}} L\left(\boldsymbol{x}^{\prime}\right) q(\breve{L})
$$

or

$$
a(\boldsymbol{R})=-\sum_{\mathcal{M}} L(\boldsymbol{R}) q(\breve{L})
$$

respectively.

In case $a$ depends on both $\boldsymbol{x}^{\prime}$ and $\boldsymbol{R}$ splitting it into two factors, one depending on $\boldsymbol{x}^{\prime}$ and one on $\boldsymbol{R}$, is possible under two different conditions:

1. All loops $L \in \mathcal{M}$ are pairwise connected and contain the same set of traits $\breve{\boldsymbol{x}}_{\mathcal{M}}^{\prime}$. Then

$$
a\left(\boldsymbol{x}^{\prime}, \boldsymbol{R}\right)=\prod_{\breve{\boldsymbol{x}}_{\mathcal{M}}^{\prime}} x_{i}^{\prime} \sum_{\mathcal{M}}\left[L / \boldsymbol{x}_{L}^{\prime}\right](\boldsymbol{R})
$$




$$
a\left(\boldsymbol{x}^{\prime}, \boldsymbol{R}\right)=\prod_{\breve{\boldsymbol{x}}_{\mathcal{M}}^{\prime}} x_{i}^{\prime} \sum_{\mathcal{M}}\left[L / \boldsymbol{x}_{L}^{\prime}\right](\boldsymbol{R}) q(\breve{L})(\boldsymbol{R}),
$$

where the first equation applies to disjunct sets of loops while the second equation applies to intersecting sets of loops.

2. All loops $L \in \mathcal{M}$ are pairwise connected and regulated in the same way. By the latter we mean that the corresponding products of regulatory functions are identical as function of $\hat{\boldsymbol{E}}: \prod_{\breve{\boldsymbol{R}}_{L}} R_{\mathrm{z}, l k}=\prod_{\breve{\boldsymbol{R}}_{M}} R_{\mathrm{z}, l k}$ for all loops $L, M \in \mathcal{M}$. This can be realized because either all loops $L \in \mathcal{M}$ contain the same set of regulated demographic parameters or, in cases that different demographic parameters are regulated, the corresponding regulatory functions are identical. Then

$$
\begin{aligned}
& a\left(\boldsymbol{x}^{\prime}, \boldsymbol{R}\right)=\prod_{\breve{\boldsymbol{R}}_{\mathcal{M}}} R_{\mathrm{z}, l k} \sum_{\mathcal{M}}\left[L / \boldsymbol{R}_{L}\right]\left(\boldsymbol{x}^{\prime}\right) \\
& a\left(\boldsymbol{x}^{\prime}, \boldsymbol{R}\right)=\prod_{\breve{\boldsymbol{R}}_{\mathcal{M}}} R_{\mathrm{z}, l k} \sum_{\mathcal{M}}\left[L / \boldsymbol{R}_{L}\right]\left(\boldsymbol{x}^{\prime}\right) q(\breve{L})\left(\boldsymbol{x}^{\prime}\right),
\end{aligned}
$$

where the first equation applies to disjunct sets of loops while the second equation applies to intersecting sets of loops.

The second group, $b$, can be written as the product of two factors if two complementary index sets $\alpha$ and $\beta$ exist, i.e., $\alpha \cup \beta=\{1, \ldots, n\}$ and $\alpha \cap \beta=\emptyset$, such that for all loops $L \in \mathcal{L}_{\mathrm{A}} \backslash \mathcal{M}$ holds that $\breve{L} \subseteq \alpha$ or $\breve{L} \subseteq \beta$. Then

$$
\begin{aligned}
& q(\alpha)=-1+\sum_{\mathcal{L}_{\mathrm{A}_{\alpha}}} L-\sum_{\mathcal{L}_{\mathrm{A}_{\alpha}}^{2 *}} L M+\sum_{\mathcal{L}_{\mathrm{A}_{\alpha}}^{3 *}} L M N-\cdots \\
& q(\beta)=-1+\sum_{\mathcal{L}_{\mathrm{A}_{\beta}}} L-\sum_{\mathcal{L}_{\mathrm{A}_{\beta}}^{2 *}} L M+\sum_{\mathcal{L}_{\mathrm{A}_{\beta}}^{3 *}} L M N-\cdots
\end{aligned}
$$

and

$$
b=-q(\alpha) q(\beta) .
$$

If loops $L \in \mathcal{L}_{\mathrm{A}_{\alpha}}$ only depend on $\boldsymbol{x}$ while loops $L \in \mathcal{L}_{\mathrm{A}_{\beta}}$ only depend on $\boldsymbol{R}$, then $b\left(\boldsymbol{x}^{\prime}, \boldsymbol{R}\right)=q(\alpha)\left(\boldsymbol{x}^{\prime}\right) q(\beta)(\boldsymbol{R})$.

We now have the necessary ingredients to apply Proposition 10 . The cases where loops can be grouped into disjunct or intersecting sets of loops can both be combined with the patterns described in Eq. (16a)-(19). This results in the following 16 optimisation principles. Note that throughout this list we make use of Lemma 1 and Corollary 2.

\section{Disjunct sets of loops}

(a) $Q=a\left(\boldsymbol{x}^{\prime}\right)+b(\boldsymbol{R})$

Optimisation principle: $\psi(\boldsymbol{x})=a(\boldsymbol{x})$

Example: Consider the life cycle in Fig. $4 \mathrm{~b}$ with $Q$ as in Eq. (12b). If $\mathcal{M}=\left\{\tilde{f}_{13} \tilde{s}_{32} \tilde{s}_{21}\right\}, \boldsymbol{x}=\left(s_{21}, s_{32}, f_{13}\right)$ and $\boldsymbol{R}=\left(R_{\mathrm{s}, 11}, R_{\mathrm{s}, 22}, R_{\mathrm{S}, 33}\right)$, then $a=f_{13}^{\prime} s_{32}^{\prime} s_{21}^{\prime}$ and $b=-\left(1-s_{11} R_{\mathrm{s}, 11}\right)\left(1-s_{22} R_{\mathrm{s}, 22}\right)\left(1-s_{33} R_{\mathrm{s}, 33}\right)$. 
(b) $Q=a(\boldsymbol{R})+b\left(\boldsymbol{x}^{\prime}\right)$

Optimisation principle: $\psi(\boldsymbol{x})=b(\boldsymbol{x})$

Example: Consider the life cycle in Fig. 4b with $Q$ as in Eq. (12b). If $\mathcal{M}=\left\{\tilde{f}_{13} \tilde{s}_{32} \tilde{s}_{21}\right\}, \boldsymbol{x}=\left(s_{11}, s_{22}, s_{33}\right)$ and $\boldsymbol{R}=\left(R_{\mathrm{s}, 21}, R_{\mathrm{s}, 32}, R_{\mathrm{f}, 13}\right)$, then $a=f_{13} R_{\mathrm{f}, 13} s_{32} R_{\mathrm{s}, 32} s_{21} R_{\mathrm{s}, 21}$ and $b=-\left(1-s_{11}^{\prime}\right)\left(1-s_{22}^{\prime}\right)\left(1-s_{33}^{\prime}\right)$.

(c) $Q=a\left(\boldsymbol{x}^{\prime}\right)+b\left(\boldsymbol{x}^{\prime}, \boldsymbol{R}\right)=a\left(\boldsymbol{x}^{\prime}\right)-q(\alpha)\left(\boldsymbol{x}^{\prime}\right) q(\beta)(\boldsymbol{R})$

Optimisation principle: $\psi(\boldsymbol{x})=-a(\boldsymbol{x}) / q(\alpha)(\boldsymbol{x})$

Example: Consider the life cycle in Fig. $4 \mathrm{~b}$ with $Q$ as in Eq. (12b). If $\mathcal{M}=$ $\left\{\tilde{f}_{13} \tilde{s}_{32} \tilde{s}_{21}\right\}, \boldsymbol{x}=\left(s_{21}, s_{32}, s_{33}, f_{13}\right)$ and $\boldsymbol{R}=\left(R_{\mathrm{s}, 11}, R_{\mathrm{s}, 22}\right)$, then $a\left(\boldsymbol{x}^{\prime}\right)=$ $f_{13}^{\prime} s_{32}^{\prime} s_{21}^{\prime}, q(\alpha)\left(\boldsymbol{x}^{\prime}\right)=-\left(1-s_{33}^{\prime}\right)$ and $q(\beta)(\boldsymbol{R})=-\left(1-s_{11} R_{\mathrm{s}, 11}\right)(1-$ $\left.s_{22} R_{\mathrm{s}, 22}\right)$.

(d) $Q=a(\boldsymbol{R})+b\left(\boldsymbol{x}^{\prime}, \boldsymbol{R}\right)=a(\boldsymbol{R})-q(\alpha)\left(\boldsymbol{x}^{\prime}\right) q(\beta)(\boldsymbol{R})$

Optimisation principle: $\psi(\boldsymbol{x})=q(\alpha)(\boldsymbol{x})$

Example: Consider the life cycle in Fig. $4 \mathrm{~b}$ with $Q$ as in Eq. (12b). If $\mathcal{M}=\left\{\tilde{f}_{13} \tilde{s}_{32} \tilde{s}_{21}\right\}, \boldsymbol{x}=\left(s_{11}, s_{22}\right)$ and $\boldsymbol{R}=\left(R_{\mathrm{s}, 21}, R_{\mathrm{s}, 32}, R_{\mathrm{s}, 33}, R_{\mathrm{f}, 13}\right)$, then $a(\boldsymbol{R})=f_{13} R_{\mathrm{f}, 13} s_{32} R_{\mathrm{s}, 32 s_{21}} R_{\mathrm{s}, 21}, q(\alpha)\left(\boldsymbol{x}^{\prime}\right)=-\left(1-s_{11}^{\prime}\right)\left(1-s_{22}^{\prime}\right)$ and $q(\beta)(\boldsymbol{R})=-\left(1-s_{33} R_{\mathrm{s}, 33}\right)$.

(e) $Q=a\left(\boldsymbol{x}^{\prime}, \boldsymbol{R}\right)+b\left(\boldsymbol{x}^{\prime}\right)=\prod_{\breve{x}_{\mathcal{M}}^{\prime}} x_{j}^{\prime} \sum_{\mathcal{M}}\left[L / \boldsymbol{x}_{L}^{\prime}\right](\boldsymbol{R})+b\left(\boldsymbol{x}^{\prime}\right)$

Optimisation principle: $\psi(\boldsymbol{x})=\prod_{\breve{x}_{\mathcal{M}}} x_{j} /|b(\boldsymbol{x})|$

Example: Consider the life cycle in Fig. 4a with $Q$ as in Eq. (12a). If $\mathcal{M}=\left\{\tilde{f}_{13} \tilde{s}_{32} \tilde{s}_{21}, \tilde{f}_{14} \tilde{s}_{43} \tilde{s}_{32} \tilde{s}_{21}\right\}$ and $\boldsymbol{x}=\left(s_{21}, s_{32}, s_{11}\right)$, then $\prod_{\breve{x}_{\mathcal{M}}^{\prime}} x_{j}^{\prime}=$ $s_{32}^{\prime} s_{21}^{\prime}, \sum_{\mathcal{M}}\left[L / \boldsymbol{x}_{L}^{\prime}\right](\boldsymbol{R})=\tilde{f}_{13}+\tilde{f}_{14} \tilde{s}_{43}$ and $b\left(\boldsymbol{x}^{\prime}\right)=-\left(1-s_{11}^{\prime}\right)$. Then $\psi\left(\boldsymbol{x}^{\prime}\right)=s_{32}^{\prime} s_{21}^{\prime} /\left(1-s_{11}^{\prime}\right)$ as long as $s_{11}$ is not regulated.

(f) $Q=a\left(\boldsymbol{x}^{\prime}, \boldsymbol{R}\right)+b(\boldsymbol{R})=\prod_{\breve{\boldsymbol{R}}_{\mathcal{M}}} R_{j} \sum_{\mathcal{M}}\left[L / \boldsymbol{R}_{L}\right]\left(\boldsymbol{x}^{\prime}\right)+b(\boldsymbol{R})$

Optimisation principle: $\psi(\boldsymbol{x})=\sum_{\mathcal{M}}\left[L / \boldsymbol{R}_{L}\right](\boldsymbol{x})$

Example: Consider the life cycle in Fig. 4 a with $Q$ as in Eq. (12a). If $\mathcal{M}=\left\{\tilde{f}_{13} \tilde{s}_{32} \tilde{s}_{21}, \tilde{f}_{14} \tilde{s}_{43} \tilde{s}_{32} \tilde{s}_{21}\right\}, \boldsymbol{x}=\left(s_{21}, s_{32}, s_{43}, f_{13}, f_{14}\right)$ and $\boldsymbol{R}=$ $\left(R_{\mathrm{S}, 21}, R_{\mathrm{S}, 32}, R_{\mathrm{s}, 11}\right)$, then $\prod_{\breve{R}_{\mathcal{M}}} R_{j}=R_{\mathrm{S}, 21} R_{\mathrm{S}, 32}, \sum_{\mathcal{M}}\left[L / \boldsymbol{R}_{L}\right]\left(\boldsymbol{x}^{\prime}\right)=$ $\left(f_{13}+f_{14} s_{43}\right) s_{32} s_{21}$ and $b(\boldsymbol{R})=-\left(1-s_{11} R_{\mathrm{S}, 11}\right)$.

(g) $Q=a\left(\boldsymbol{x}^{\prime}, \boldsymbol{R}\right)+b\left(\boldsymbol{x}^{\prime}, \boldsymbol{R}\right)=\prod_{\breve{x}_{\mathcal{M}}^{\prime}} x_{j}^{\prime} \sum_{\mathcal{M}}\left[L / \boldsymbol{x}_{L}^{\prime}\right](\boldsymbol{R})-q(\alpha)\left(\boldsymbol{x}^{\prime}\right) q(\beta)(\boldsymbol{R})$ Optimisation principle: $\psi(\boldsymbol{x})=-\prod_{\breve{x}_{\mathcal{M}}} x_{j} / q(\alpha)(\boldsymbol{x})$

Example: Consider the life cycle in Fig. $4 \mathrm{~b}$ with $Q$ as in Eq. (12b). If $\mathcal{M}=$ $\left\{\tilde{f}_{13} \tilde{s}_{32} \tilde{s}_{21}\right\}, \boldsymbol{R}=\left(R_{\mathrm{s}, 21}, R_{\mathrm{s}, 32}, R_{\mathrm{s}, 11}, R_{\mathrm{s}, 22}, R_{\mathrm{f}, 13}\right)$ and $\boldsymbol{x}=\left(s_{21}, s_{32}, s_{33}\right.$, $\left.f_{13}\right)$, then $\prod_{\breve{x}_{\mathcal{M}}^{\prime}} x_{j}^{\prime}=f_{13}^{\prime} s_{32}^{\prime} s_{21}^{\prime}, \sum_{\mathcal{M}}\left[L / x_{L}^{\prime}\right](\boldsymbol{R})=R_{\mathrm{f}, 13} R_{\mathrm{s}, 32} R_{\mathrm{s}, 21}, q(\alpha)$ $\left(\boldsymbol{x}^{\prime}\right)=-\left(1-s_{33}^{\prime}\right)$ and $q(\beta)(\boldsymbol{R})=-\left(1-s_{11} R_{\mathrm{S}, 11}\right)\left(1-s_{22} R_{\mathrm{S}, 22}\right)$.

(h) $Q=a\left(\boldsymbol{x}^{\prime}, \boldsymbol{R}\right)+b\left(\boldsymbol{x}^{\prime}, \boldsymbol{R}\right)=\prod_{\breve{\boldsymbol{R}}_{\mathcal{M}}} R_{j} \sum_{\mathcal{M}}\left[L / \boldsymbol{R}_{L}\right]\left(\boldsymbol{x}^{\prime}\right)-q(\alpha)\left(\boldsymbol{x}^{\prime}\right) q(\beta)(\boldsymbol{R})$ Optimisation principle: $\psi(\boldsymbol{x})=-\sum_{\mathcal{M}}\left[L / \boldsymbol{R}_{L}\right](\boldsymbol{x}) / q(\alpha)(\boldsymbol{x})$

Example: The preceding example also fits in the present case.

\section{Intersecting sets of loops}

(a) $Q=a\left(\boldsymbol{x}^{\prime}\right)+b(\boldsymbol{R})=-\sum_{\mathcal{M}} L\left(\boldsymbol{x}^{\prime}\right) q(\breve{L})+b(\boldsymbol{R})$

Optimisation principle: $\psi(\boldsymbol{x})=-\sum_{\mathcal{M}} L(\boldsymbol{x}) q(\breve{L})$ 
Example: Consider the life cycle in Fig. $4 \mathrm{c}$ with $Q$ as in Eq. (12c). If $\mathcal{M}=\left\{\tilde{f}_{13} \tilde{s}_{32} \tilde{s}_{21}, \tilde{f}_{14} \tilde{s}_{43} \tilde{s}_{32} \tilde{s}_{21}\right\}, \boldsymbol{x}=\left(s_{21}, s_{32}, s_{43}, f_{13}, f_{14}\right)$ and $\boldsymbol{R}=$ $\left(R_{\mathrm{s}, 22}, R_{\mathrm{s}, 33}\right)$, then $a=\left(f_{13}^{\prime}\left(1-s_{44}\right)+f_{14}^{\prime} s_{43}^{\prime}\right) s_{32}^{\prime} s_{21}^{\prime}\left(1-s_{55}\right)$ and $b=$ $-\left(1-s_{22} R_{\mathrm{s}, 22}\right)\left(1-s_{33} R_{\mathrm{s}, 33}\right)\left(1-s_{44}\right)\left(1-s_{55}\right)$.

(b) $Q=a(\boldsymbol{R})+b\left(\boldsymbol{x}^{\prime}\right)=-\sum_{\mathcal{M}} L(\boldsymbol{R}) q(\breve{L})+b\left(\boldsymbol{x}^{\prime}\right)$

Optimisation principle: $\psi(\boldsymbol{x})=b(\boldsymbol{x})$

Example: Consider the life cycle in Fig. 4c with $Q$ as in Eq. (12c). If $\mathcal{M}=\left\{\tilde{f}_{13} \tilde{s}_{32} \tilde{s}_{21}, \tilde{f}_{14} \tilde{s}_{43} \tilde{s}_{32} \tilde{s}_{21}\right\}, \boldsymbol{x}=\left(s_{22}, s_{33}\right)$ and $\boldsymbol{R}=\left(R_{\mathrm{f}, 13}, R_{\mathrm{f}, 14}\right)$, then $b=-\left(1-s_{22}^{\prime}\right)\left(1-s_{33}^{\prime}\right)\left(1-s_{44}\right)\left(1-s_{55}\right)$ and $a=\left(f_{13} R_{\mathrm{f}, 13}(1-\right.$

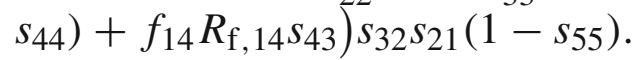

(c) $Q=a\left(\boldsymbol{x}^{\prime}, \boldsymbol{R}\right)+b(\boldsymbol{R})=-\prod_{\breve{x}_{\mathcal{M}}^{\prime}} x_{j}^{\prime} \sum_{\mathcal{M}}\left[L / \boldsymbol{x}_{L}^{\prime}\right](\boldsymbol{R}) q(\breve{L})(\boldsymbol{R})+b(\boldsymbol{R})$

Optimisation principle: $\psi(\boldsymbol{x})=\prod_{\breve{x}_{\mathcal{M}}} x_{j}$

Example: Consider the life cycle in Fig. 4c with $Q$ as in Eq. (12c). If $\mathcal{M}=\left\{\tilde{f}_{13} \tilde{s}_{32} \tilde{s}_{21}, \tilde{f}_{14} \tilde{s}_{43} \tilde{s}_{32} \tilde{s}_{21}\right\}$ and $\boldsymbol{x}=\left(s_{21}, s_{32}\right)$, then $\prod_{\breve{x}_{\mathcal{M}}^{\prime}} x_{j}^{\prime}=s_{32}^{\prime} s_{21}^{\prime}$, $\sum_{\mathcal{M}}\left[L / \boldsymbol{x}_{L}^{\prime}\right](\boldsymbol{R}) q(\breve{L})(\boldsymbol{R})=\left(f_{13}\left(1-s_{44}\right)+f_{14} s_{43}\right)\left(1-s_{55}\right)$ and $b(\boldsymbol{R})=$ $-\left(1-\tilde{s}_{22}\right)\left(1-\tilde{s}_{33}\right)\left(1-\tilde{s}_{44}\right)\left(1-\tilde{s}_{55}\right)$. Then $\psi(\boldsymbol{x})=s_{21} s_{32}$ regardless of the set of regulatory functions.

(d) $Q=a\left(\boldsymbol{x}^{\prime}, \boldsymbol{R}\right)+b\left(\boldsymbol{x}^{\prime}\right)=-\prod_{\breve{R}_{\mathcal{M}}} R_{j} \sum_{\mathcal{M}}\left[L / \boldsymbol{R}_{L}\right]\left(\boldsymbol{x}^{\prime}\right) q(\breve{L})\left(\boldsymbol{x}^{\prime}\right)+b\left(\boldsymbol{x}^{\prime}\right)$

Optimisation principle: $\psi(\boldsymbol{x})=\sum_{\mathcal{M}}\left[L / \boldsymbol{R}_{L}\right](\boldsymbol{x}) q(\breve{L})(\boldsymbol{x}) /|b(\boldsymbol{x})|$

Example: Consider the life cycle in Fig. $4 \mathrm{c}$ with $Q$ as in Eq. (12c). If $\mathcal{M}=\left\{\tilde{f}_{13} \tilde{s}_{32} \tilde{s}_{21}, \tilde{f}_{14} \tilde{s}_{43} \tilde{s}_{32} \tilde{s}_{21}\right\}$ and $\boldsymbol{R}=\left(R_{\mathrm{f}, 13}, R_{\mathrm{f}, 14}\right)$ with $R_{\mathrm{f}, 13}=R=$ $R_{\mathrm{f}, 14}$, then $\prod_{\breve{R}_{\mathcal{M}}} R_{j}=R, \sum_{\mathcal{M}}\left[L / \boldsymbol{R}_{L}\right]\left(\boldsymbol{x}^{\prime}\right) q(\breve{L})\left(\boldsymbol{x}^{\prime}\right)=\left(f_{13}^{\prime}\left(1-s_{44}^{\prime}\right)+\right.$ $\left.f_{14}^{\prime} s_{43}^{\prime}\right) s_{32}^{\prime} s_{21}^{\prime}\left(1-s_{55}^{\prime}\right)$ and $b\left(\boldsymbol{x}^{\prime}\right)=-\left(1-s_{22}^{\prime}\right)\left(1-s_{33}^{\prime}\right)\left(1-s_{44}^{\prime}\right)\left(1-s_{55}^{\prime}\right)$.

(e) $Q=a\left(\boldsymbol{x}^{\prime}\right)+b\left(\boldsymbol{x}^{\prime}, \boldsymbol{R}\right)=-\sum_{\mathcal{M}} L\left(\boldsymbol{x}^{\prime}\right) q(\breve{L})\left(\boldsymbol{x}^{\prime}\right)-q(\alpha)\left(\boldsymbol{x}^{\prime}\right) q(\beta)(\boldsymbol{R})$

Optimisation principle: $\psi(\boldsymbol{x})=\sum_{\mathcal{M}} L(\boldsymbol{x}) q(\breve{L})(\boldsymbol{x}) / q(\alpha)(\boldsymbol{x})$

Example: Consider the life cycle in Fig. $4 \mathrm{c}$ with $Q$ as in Eq. (12c). If $\mathcal{M}=$ $\left\{\tilde{f}_{13} \tilde{s}_{32} \tilde{s}_{21}, \tilde{f}_{14} \tilde{s}_{43} \tilde{s}_{32} \tilde{s}_{21}, \boldsymbol{x}=\left(s_{21}, s_{32}, s_{43}, s_{44}, s_{55}, f_{13}, f_{14}\right)\right\}$ and $\boldsymbol{R}=$ $\left(R_{\mathrm{s}, 22}, R_{\mathrm{s}, 33}\right)$, then $\sum_{\mathcal{M}} L\left(\boldsymbol{x}^{\prime}\right) q(\breve{L})\left(\boldsymbol{x}^{\prime}\right)=\left(f_{13}^{\prime}\left(1-s_{44}^{\prime}\right)+f_{14}^{\prime} s_{43}^{\prime}\right) s_{32}^{\prime} s_{21}^{\prime}$ $\left(1-s_{55}^{\prime}\right), q(\alpha)\left(\boldsymbol{x}^{\prime}\right)=-\left(1-s_{44}^{\prime}\right)\left(1-s_{55}^{\prime}\right)$ and $q(\beta)(\boldsymbol{R})=-\left(1-s_{22} R_{\mathrm{s}, 22}\right)$ $\left(1-s_{33} R_{\mathrm{s}, 33}\right)$.

(f) $Q=a(\boldsymbol{R})+b\left(\boldsymbol{x}^{\prime}, \boldsymbol{R}\right)=-\sum_{\mathcal{M}} L(\boldsymbol{R}) q(\breve{L})(\boldsymbol{R})-q(\alpha)\left(\boldsymbol{x}^{\prime}\right) q(\beta)(\boldsymbol{R})$

Optimisation principle: $\psi(\boldsymbol{x})=q(\alpha)(\boldsymbol{x})$

Example: Consider the life cycle in Fig. $4 \mathrm{c}$ with $Q$ as in Eq. (12c). If $\mathcal{M}=$ $\left\{\tilde{f}_{13} \tilde{s}_{32} \tilde{s}_{21}, \tilde{f}_{14} \tilde{s}_{43} \tilde{s}_{32} \tilde{s}_{21}\right\}$ and $\boldsymbol{x}=\left(s_{22}, s_{33}\right)$, then $\sum_{\mathcal{M}} L(\boldsymbol{R}) q(\breve{L})(\boldsymbol{R})=$ $-\left(\tilde{f}_{13}\left(1-\tilde{s}_{44}\right)+\tilde{f}_{14} \tilde{s}_{43}\right) \tilde{s}_{32} \tilde{s}_{21}\left(1-\tilde{s}_{55}\right), q(\alpha)\left(\boldsymbol{x}^{\prime}\right)=-\left(1-s_{22}^{\prime}\right)\left(1-s_{33}^{\prime}\right)$ and $q(\beta)(\boldsymbol{R})=-\left(1-\tilde{s}_{44}\right)\left(1-\tilde{s}_{55}\right)$. Then $\psi(\boldsymbol{x})=-\left(1-s_{22}\right)\left(1-s_{33}\right)$ as long as $s_{22}$ and $s_{33}$ are not regulated.

(g) $Q=a\left(\boldsymbol{x}^{\prime}, \boldsymbol{R}\right)+b\left(\boldsymbol{x}^{\prime}, \boldsymbol{R}\right)=-\prod_{\breve{x}_{\mathcal{M}}^{\prime}} x_{j}^{\prime} \sum_{\mathcal{M}}\left[L / \boldsymbol{x}_{L}^{\prime}\right](\boldsymbol{R}) q(\breve{L})(\boldsymbol{R})-q(\alpha)$ $\left(\boldsymbol{x}^{\prime}\right) q(\beta)(\boldsymbol{R})$

Optimisation principle: $\psi(\boldsymbol{x})=-\prod_{\breve{x}_{\mathcal{M}}} x_{j} / q(\alpha)(\boldsymbol{x})$ 
Example: Consider the life cycle in Fig. $4 \mathrm{c}$ with $Q$ as in Eq. (12c). If $\mathcal{M}=$ $\left\{\tilde{f}_{13} \tilde{s}_{32} \tilde{s}_{21}, \tilde{f}_{14} \tilde{s}_{43} \tilde{s}_{32} \tilde{s}_{21}\right\}$ and $\boldsymbol{x}=\left(s_{21}, s_{32}, s_{22}, s_{33}\right)$, then $\sum_{\mathcal{M}}\left[L / \boldsymbol{x}_{L}^{\prime}\right]$ $(\boldsymbol{R}) q(\breve{L})(\boldsymbol{R})=\left(\tilde{f}_{13}\left(1-\tilde{s}_{44}\right)+\tilde{f}_{14} \tilde{s}_{43}\right)\left(1-\tilde{s}_{55}\right), q(\alpha)\left(\boldsymbol{x}^{\prime}\right)=-\left(1-s_{22}^{\prime}\right)(1-$ $\left.s_{33}^{\prime}\right)$ and $q(\beta)(\boldsymbol{R})=-\left(1-\tilde{s}_{44}\right)\left(1-\tilde{s}_{55}\right)$. Then $\psi(\boldsymbol{x})=-s_{21} s_{32} /(1-$ $\left.s_{22}\right)\left(1-s_{33}\right)$ as long as $s_{22}$ and $s_{33}$ are not regulated.

(h) $Q=a\left(\boldsymbol{x}^{\prime}, \boldsymbol{R}\right)+b\left(\boldsymbol{x}^{\prime}, \boldsymbol{R}\right)=-\prod_{\breve{R}_{\mathcal{M}}} R_{j} \sum_{\mathcal{M}}\left[L / \boldsymbol{R}_{L}\right]\left(\boldsymbol{x}^{\prime}\right) q(\breve{L})\left(\boldsymbol{x}^{\prime}\right)-q(\alpha)$ $\left(\boldsymbol{x}^{\prime}\right) q(\beta)(\boldsymbol{R})$

Optimisation principle: $\psi(\boldsymbol{x})=\sum_{\mathcal{M}}\left[L / \boldsymbol{R}_{L}\right](\boldsymbol{x}) q(\breve{L})(\boldsymbol{x}) / q(\alpha)(\boldsymbol{x})$

Example: Consider the life cycle in Fig. 4c with $Q$ as in Eq. (12c). If $\mathcal{M}=\left\{\tilde{f}_{13} \tilde{s}_{32} \tilde{s}_{21}, \tilde{f}_{14} \tilde{s}_{43} \tilde{s}_{32} \tilde{s}_{21}\right\}$ and $\boldsymbol{R}=\left(R_{\mathrm{s}, 21}, R_{\mathrm{S}, 32}, R_{\mathrm{S}, 22}, R_{\mathrm{S}, 33}\right)$, then $\prod_{\breve{R}_{\mathcal{M}}} R_{j}=R_{\mathrm{s}, 32} R_{\mathrm{s}, 21}, \sum_{\mathcal{M}}\left[L / \boldsymbol{R}_{L}\right]\left(\boldsymbol{x}^{\prime}\right) q(\breve{L})\left(\boldsymbol{x}^{\prime}\right)=-\left(f_{13}^{\prime}\left(1-s_{44}^{\prime}\right)+\right.$ $\left.f_{14}^{\prime} s_{43}^{\prime}\right) s_{32}^{\prime} s_{21}^{\prime}\left(1-s_{55}^{\prime}\right), q(\alpha)\left(\boldsymbol{x}^{\prime}\right)=-\left(1-s_{44}^{\prime}\right)\left(1-s_{55}^{\prime}\right)$ and $q(\beta)(\boldsymbol{R})=$ $-\left(1-s_{22} R_{\mathrm{s}, 22}\right)\left(1-s_{33} R_{\mathrm{s}, 33}\right)$.

\subsection{Other optimisation principles}

Here we collect three optimisation principles that are not based on $Q$. First we consider the case that an optimisation principle can be derived from a one-dimensionally acting environment.

Proposition 13 If there exist functions $\phi: \mathscr{E} \rightarrow \mathbb{R}$ and $h_{\mathrm{z}, l k}: \mathbb{R} \rightarrow \mathbb{R}$ such that all regulatory functions $R_{\mathrm{z}, l k}$ can be written as $R_{\mathrm{z}, l k}=h_{\mathrm{z}, l k} \circ \phi$ with $h_{\mathrm{z}, l k}$ monotonically increasing in $\phi(\hat{\boldsymbol{E}})$, then $\phi$ will be minimized and e.g. $\psi_{1}: \mathscr{X} \rightarrow \mathbb{R}: \psi_{1}(\boldsymbol{x}) \mapsto$ $-\phi(\hat{\boldsymbol{E}}(\boldsymbol{x}))$ and $\psi_{2}: \mathscr{X} \rightarrow \mathbb{R}: \psi_{2}(\boldsymbol{x}) \mapsto 1 / \phi(\hat{\boldsymbol{E}}(\boldsymbol{x}))$ will be maximized.

Proof Since the dominant eigenvalue of a non-negative matrix is strictly increasing in all matrix entries (Horn and Johnson 1985), it follows that $\lambda_{\mathrm{d}}\left(\boldsymbol{x}^{\prime}, \hat{\boldsymbol{E}}\right)$ is monotonically related to $\phi(\hat{\boldsymbol{E}})$. By choosing $\beta\left(\boldsymbol{x}^{\prime}, \phi(\hat{\boldsymbol{E}})\right)=\ln \lambda_{\mathrm{d}}\left(\boldsymbol{x}^{\prime}, \phi(\hat{\boldsymbol{E}})\right)$ the statement follows from Proposition 8.

Corollary 14 If $\hat{\boldsymbol{E}}$ is a scalar-valued, then we can choose $\phi=\mathrm{id}$.

The result from Corollary 14 on the minimization of $\hat{\boldsymbol{E}}$ is a generalisation of earlier findings. Several authors showed that when populations are regulated by a single resource the abundance of that resource will be minimised (Powell 1958; Armstrong and McGehee 1980; Mylius and Diekmann 1995) and, when population regulation occurs through the density of individuals in a single critical i-state, the density of individuals in that critical i-state will be maximised (Takada and Nakajima 1992, 1998; Charlesworth 1994).

The following proposition, which can be found in Mylius and Diekmann (1995) and Metz et al. (2008a), gives sufficient conditions for evolution to maximize the dominant eigenvalue of the population projection matrix $A$. We say that two demographic parameters $\tilde{z}_{j i}$ and $\tilde{z}_{l k}$ are regulated in the same way when $R_{\mathrm{Z}, j i}=R=R_{\mathrm{z}, l k}$.

Proposition 15 If all demographic parameters are regulated in the same way, then evolution maximizes $\lambda_{\mathrm{d}}(\boldsymbol{x})$ and minimizes $R(\hat{\boldsymbol{E}})$, where $\lambda_{\mathrm{d}}(\boldsymbol{x})$ denotes the dominant eigenvalue of the population projection matrix where all regulatory functions are set equal to one. 
Fig. 5 Life cycle of a semelparous plant with seed bank. Seeds are stored at different soil depths (top (1), middle (2) or deep (3)) with all seeds entering at the top. Seeds give rise to seedlings (4), which either grow into small (5) or large flowering plants (6)

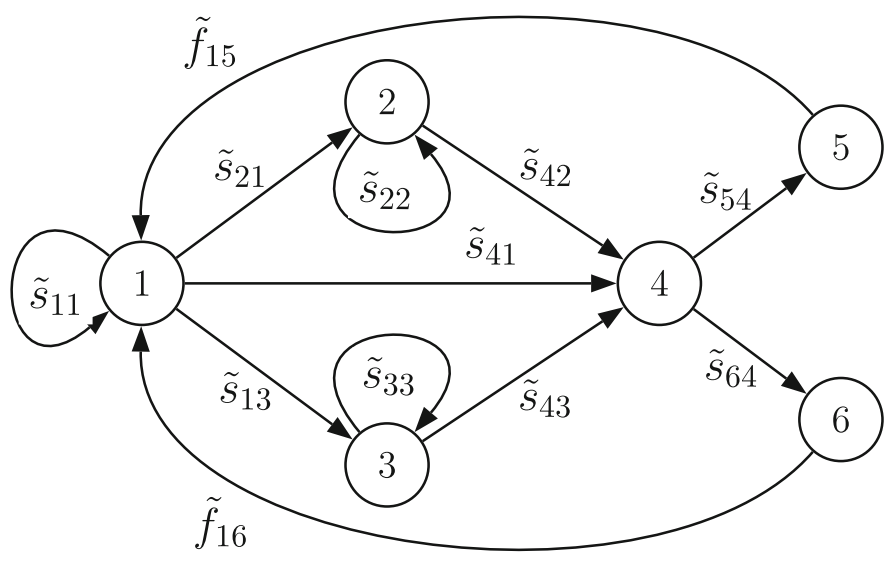

Proof Given the assumption we have $\mathrm{A}\left(\boldsymbol{x}^{\prime}, \hat{\boldsymbol{E}}\right)=R(\hat{\boldsymbol{E}}) \mathrm{A}\left(\boldsymbol{x}^{\prime}\right)$, where $\mathrm{A}\left(\boldsymbol{x}^{\prime}\right)$ denotes the population projection matrix where all regulatory functions are set equal to one. Thus $\lambda_{\mathrm{d}}\left(\boldsymbol{x}^{\prime}, \hat{\boldsymbol{E}}\right)=\lambda_{\mathrm{d}}\left(\boldsymbol{x}^{\prime}\right) R(\hat{\boldsymbol{E}})$. The statement follows from Proposition 7 and Proposition 8 by choosing $\psi\left(\boldsymbol{x}^{\prime}\right)=\lambda_{\mathrm{d}}\left(\boldsymbol{x}^{\prime}\right), \phi(\hat{\boldsymbol{E}})=R(\hat{\boldsymbol{E}})$ and $\alpha\left(\psi\left(\boldsymbol{x}^{\prime}\right), \hat{\boldsymbol{E}}\right)=$ $\ln \left[\lambda_{\mathrm{d}}\left(\boldsymbol{x}^{\prime}\right) R(\hat{\boldsymbol{E}})\right]=\beta\left(\boldsymbol{x}^{\prime}, \phi(\hat{\boldsymbol{E}})\right)$.

The condition of Proposition 15 is fulfilled when the regulation is only through mortality that affects everybody equally, including the newborns, independent of state or type.

The last proposition about optimisation is related to the basic reproduction ratio $R_{0}$. This result is a generalization of a statement formulated by Metz et al. (2008a) and applied in Metz et al. (2009). The conditions leading to this optimisation principle are rather involved and we start with an example. Consider a semelparous plant with seed bank and a life cycle as depicted in Fig. 5. The seed bank is discretised into three layers, a top layer (1), a middle layer (2) and a deep layer (3). Seeds enter the seed bank at the top layer. They stay in the top layer with probability $\tilde{s}_{11}$ or are moved to the deeper layers with probability $\tilde{s}_{21}$ or $\tilde{s}_{31}$, respectively, where they stay with probability $\tilde{s}_{22}$ or $\tilde{s}_{33}$, respectively. Depending on their position, seeds germinate and turn into seedlings (4) with probability $\tilde{s}_{41}, \tilde{s}_{42}$ or $\tilde{s}_{43}$, respectively. Seedlings turn into small flowering plants (5) with probability $\tilde{s}_{54}$ and into large flowering plants (6) with probability $\tilde{s}_{64}$. Depending on their size flowering plants produce $\tilde{f}_{15}$ or $\tilde{f}_{16}$ seeds. The expected life time number of seeds in stage (1) produced by a seed starting out in this stage equals

$$
R_{0}=\left(\tilde{f}_{15} \tilde{s}_{54}+\tilde{f}_{16} \tilde{s}_{64}\right)\left(\frac{\tilde{s}_{41}}{1-\tilde{s}_{11}}+\frac{\tilde{s}_{42} \tilde{s}_{21}}{\left(1-\tilde{s}_{11}\right)\left(1-\tilde{s}_{22}\right)}+\frac{\tilde{s}_{43} \tilde{s}_{31}}{\left(1-\tilde{s}_{11}\right)\left(1-\tilde{s}_{33}\right)}\right)
$$

Thus, $R_{0}$ can be written as a product of two factors containing disjunct sets of demographic parameters. The terms in the second bracket describe the flow of individuals from i-state (1) to i-state (4) while the terms in the first bracket describe the flow of individuals from i-state (4) to i-state (1). The product-structure of $R_{0}$ results from the life cycle in Fig. 5 having two i-states, (1) and (4), that have to be passed by every individual in order to complete the life cycle. In this model, optimisation is possible 
whenever in Eq. (20) one factor only depends on traits $\boldsymbol{x}^{\prime}$ while the other factor only depends on $\hat{\boldsymbol{E}}$ through the action of the regulatory functions. Then the factor depending on traits is an optimisation principle while the factor depending on regulatory functions is a pessimization principle, a result that follows directly from Proposition 7 and 8 by choosing $\alpha=R_{0}=\beta$ and $\psi=$ factor depending on traits and $\phi=$ factor depending on regulatory functions, respectively.

Next we present the conditions that allow to write $R_{0}$ as a product of two functions, one depending on traits and one on regulatory functions.

Proposition 16 Assume a life cycle where the $i$-states can be partitioned into $m$ disjunct classes $G_{i}, i \in \mathbb{N} \bmod m, m$ even, with each class only connecting to the following class through a single $i$-state. These obligatory $i$-states can only be traversed in one direction and are an element of the entered class. Optimisation is possible if the demographic parameters corresponding to arrows from $i$-states in odd numbered classes are not regulated (the unregulated classes), and the demographic parameters corresponding to arrows from $i$-states in even numbered classes are not evolving (the non-evolving classes). This applies both to arrows connecting to $i$-states in the same class and to arrows to the obligatory $i$-state in the next class.

Sketch of a proof This optimisation principle can be calculated by treating the entrance stream into any of the classes as "births" for which we calculate " $R_{0}$ ". This " $R_{0}$ " is a fitness proxy and can be written as a product of a function of the traits $\boldsymbol{x}$ times a function of $\hat{\boldsymbol{E}}$. The result then follows from Proposition 7. For a more detailed proof see Appendix B.

In the example leading to Eq. (20) the groups of i-states are $G_{1}=\{1,2,3\}$ and $G_{2}=\{4,5,6\}$. Proposition 16 can extended in several directions:

(I) Optimisation can also be possible if some of the obligatory i-states are a member of the class of i-states that is left instead of entered. In this case, it is additionally required that the demographic parameters corresponding to arrows from those obligatory i-states leaving the class satisfy the following restriction: If the class is non-evolving, then the demographic parameters corresponding to arrows to the next class are all regulated in the same way. If the class is unregulated, then the demographic parameters corresponding to arrows to the next class are non-evolving (cf. Appendix B).

For example, optimisation can still be possible if $G_{1}=\{2,3,4\}$ and $G_{2}=$ $\{5,6,1\}$ such that

$$
R_{0}=\left(\frac{\tilde{f}_{15} \tilde{s}_{54}}{1-\tilde{s}_{11}}+\frac{\tilde{f}_{16} \tilde{s}_{64}}{1-\tilde{s}_{11}}\right)\left(\tilde{s}_{41}+\frac{\tilde{s}_{42} \tilde{s}_{21}}{1-\tilde{s}_{22}}+\frac{\tilde{s}_{43} \tilde{s}_{31}}{1-\tilde{s}_{33}}\right)
$$

If $G_{1}\left(G_{2}\right)$ is the non-evolving class, then, for optimisation to be possible, it is necessary that $R_{\mathrm{S}, 54}=R_{\mathrm{s}, 64}\left(R_{\mathrm{S}, 21}=R_{\mathrm{S}, 31}=R_{\mathrm{S}, 41}\right)$. Furthermore, if $G_{1}$ $\left(G_{2}\right)$ is the non-regulated class, then $\tilde{s}_{54}$ and $\tilde{s}_{64}\left(\tilde{s}_{21}, \tilde{s}_{31}\right.$ and $\left.\tilde{s}_{41}\right)$ cannot be traits. Then in Eq. (21) the demographic parameters are non-evolving in one factor and unregulated in the other but for a term $R_{\mathrm{s}, l k}$ that is multiplied to each summand in that factor. 
(II) In Proposition 16 and Remark (I) it is assumed that the flux of individuals from one class of i-states to the next is channeled through a single obligatory i-state. In these cases the flux between classes is described by the outer product of two vectors, namely $(1,0, \ldots, 0)^{\mathrm{T}}\left(\tilde{z}_{d 1}, \ldots, \tilde{z}_{d k}\right)$ or $\left(\tilde{z}_{1 d}, \ldots, \tilde{z}_{l d}\right)^{\mathrm{T}}(0, \ldots, 0,1)$, respectively (Appendix B). In both cases the first vector has dimension $l$ and the second dimension $k$, where $l$ and $k$ are the number of $i$-states in the entered and left class, respectively. Thus, the flow of individuals between classes is described by a $l \times k$-class-transition matrix of rank 1 . Optimisation can also be possible if the flux of individuals from one class to the next occurs through more than a single i-state as long as the class-transition matrix has still rank 1, i.e., as long as two vectors $\left(v_{1 d}, \ldots, v_{l d}\right)$ and $\left(u_{d 1}, \ldots, u_{d k}\right)$ exists such that the classtransition matrix equals $\left(v_{1 d}, \ldots, v_{l d}\right)^{\mathrm{T}}\left(u_{d 1}, \ldots, u_{d k}\right)$ (Appendix B). It is then necessary that the entries in the vectors $\boldsymbol{v}$ and $\boldsymbol{u}$ are non-evolving (unregulated) and unregulated (non-evolving), respectively, if the entered class is non-evolving (unregulated) and the left class unregulated (non-evolving).

As an example consider the life cycle of Fig. 5 but without the seedling i-state (4). Thus, seeds directly develop into flowering plants between two censuses. Let us group the classes into $G_{1}=\{1,2,3\}$ and $G_{2}=\{5,6\}$. Now the transition from $G_{1}$ to $G_{2}$ occurs through two i-states, (5) and (6), and Proposition 16 cannot be applied. If, however, each seed has the same probability to develop into a small and large flowering plant, such that $\tilde{s}_{5 j}=p \tilde{s}_{j}$ and $\tilde{s}_{6 j}=(1-p) \tilde{s}_{j}$ for $p \in[0,1]$ and $j \in\{1,2,3\}$, then

$$
\begin{aligned}
R_{0}= & \left(p \tilde{f}_{15}+(1-p) \tilde{f}_{16}\right) \\
& \times\left(\frac{\tilde{s}_{1}}{1-\tilde{s}_{11}}+\frac{\tilde{s}_{21} \tilde{s}_{2}}{\left(1-\tilde{s}_{11}\right)\left(1-\tilde{s}_{22}\right)}+\frac{\tilde{s}_{31} \tilde{s}_{3}}{\left(1-\tilde{s}_{11}\right)\left(1-\tilde{s}_{33}\right)}\right) .
\end{aligned}
$$

Optimisation is possible if the demographic parameters in the first pair of brackets are non-evolving and those in the second pair of brackets are unregulated, or vice versa.

Class-transition matrices of rank 1 often result when the passing through a single state from one class of $i$-states to the next is hidden as it is often the case when the state space depends on the moment chosen as census. For instance, consider the case that the state "seed above ground" only occurs shortly after seed shedding. If the point of census is just a little later, seeds either have been moved below ground or have been consumed by seed eating animals. Thus, although seeds above ground may never be observed, all seeds have to pass through this i-state and the transition between the class of i-states describing the plant and the class of $i$-states describing the seed bank is described by the rank-1-matrix $\left(\tilde{s}_{2 d}, \tilde{s}_{3 d}\right)^{\mathrm{T}}\left(0, \tilde{f}_{d 5}, \tilde{f}_{d 6}\right)$, where $d$ is a dummy variable describing the hidden i-state "seed above ground".

(III) The paths through the life cycle graph that lead from an i-state where the class can be entered to an exit arrow extended with those exit arrows will be called through-paths. For instance, in the above example $\tilde{s}_{41}, \tilde{s}_{21} \tilde{s}_{42}$ and $\tilde{s}_{31} \tilde{s}_{43}$ are all through-paths of the first class of i-states. The class of models delimited in Proposition 16 can be extended by allowing for each (otherwise) non-regu- 
lated class that all through-paths are regulated in exactly the same way. By this we mean that the corresponding products of regulatory functions are identical as function of $\hat{\boldsymbol{E}}: \prod_{\breve{\boldsymbol{R}}_{L}} R_{\mathrm{z}, l k}=\prod_{\breve{\boldsymbol{R}}_{M}} R_{\mathrm{z}, l k}$ for all through paths $L$ and $M$. Then, and under the restriction that all within-class loops are free of regulated demographic parameters, the regulatory functions can be factored out from the through-paths. The members in the so enlarged class of models also have an $R_{0}$ that can be written as a function of $\boldsymbol{x}$ times a function of $\hat{\boldsymbol{E}}$.

For example, optimisation is still possible with $R_{0}$ as in Eq. (20) with $G_{1}=$ $\{1,2,3\}$ the (otherwise) unregulated class but with population regulation permitted if it fulfills one of the following conditions: $R_{\mathrm{S}, 41}=R_{\mathrm{s}, 21}=R_{\mathrm{S}, 31}$, $R_{\mathrm{s}, 41}=R_{\mathrm{s}, 42}=R_{\mathrm{s}, 31}, R_{\mathrm{s}, 41}=R_{\mathrm{s}, 21}=R_{\mathrm{s}, 43}$ or $R_{\mathrm{s}, 41}=R_{\mathrm{s}, 42}=R_{\mathrm{s}, 43}$.

\section{Frequency-dependent selection}

In Propositions 10, 13, 15 and 16 we presented conditions allowing for optimisation. If none of these propositions can be applied it is possible that the feedback environment and the trait vector are not acting in a monotone and one-dimensional manner and selection can be frequency-dependent in the sense of the definition given in Sect. 2.4. The following proposition states necessary requirements such that the trait vector and the feedback environment are not acting in a one-dimensional manner in terms of the distribution of traits and regulatory functions over the loops of a life cycle. In Sect. 7 we analyze examples where selection is indeed frequency-dependent.

Proposition 17 For selection to be frequency-dependent it is necessary that $\operatorname{dim} \mathscr{E}>$ 1 and that at least two evolving and two regulated loops exist which occur in one of the following combinations:

(i) A pair of loops $L, M$ exist that are both evolving and regulated such that

$$
Q\left(\boldsymbol{x}^{\prime}, \hat{\boldsymbol{E}}\right)=L\left(\boldsymbol{x}^{\prime}, \hat{\boldsymbol{E}}\right)+M\left(\boldsymbol{x}^{\prime}, \hat{\boldsymbol{E}}\right)+\text { rest. }
$$

(ii) Three loops $L, M, N$ exist where $L$ is both evolving and regulated, $M$ is evolving and $N$ is regulated such that

$$
Q\left(\boldsymbol{x}^{\prime}, \hat{\boldsymbol{E}}\right)=L\left(\boldsymbol{x}^{\prime}, \hat{\boldsymbol{E}}\right)+M\left(\boldsymbol{x}^{\prime}\right)+N(\hat{\boldsymbol{E}})+\text { rest. }
$$

(iii) Four loops $L, M, N$ and $O$ exist where $L$ and $M$ are evolving and $N$ and $O$ are regulated and where $L$ and $N$ are unconnected such that

$$
Q\left(\boldsymbol{x}^{\prime}, \hat{\boldsymbol{E}}\right)=L\left(\boldsymbol{x}^{\prime}\right)+M\left(\boldsymbol{x}^{\prime}\right)+N(\hat{\boldsymbol{E}})+O(\hat{\boldsymbol{E}})-L\left(\boldsymbol{x}^{\prime}\right) N(\hat{\boldsymbol{E}})+\text { rest },
$$

where $\left(\boldsymbol{x}^{\prime}\right),(\hat{\boldsymbol{E}})$ and $\left(\boldsymbol{x}^{\prime}, \hat{\boldsymbol{E}}\right)$ are added as arguments to loops to indicate whether they contain a trait, a regulated demographic parameter or both.

Proof If $\operatorname{dim} \mathscr{E}=1$ Proposition 13 and in particularly Corollary 14 apply because of the assumption that all regulatory functions are monotone in $\hat{\boldsymbol{E}}$ (cf. Sect. 2.1). If all 
traits occur in a single loop or if all regulated demographic parameters occur in a single loop, then Corollary 11(i) or (ii) applies, respectively. If no summand in $Q$ exists that is affected by at least one trait and one regulated demographic parameters, then Corollary 11(iii) applies. Taken together, this proves the necessity of $\operatorname{dim} \mathscr{E}>1$ and of the existence of at least two loops containing traits, two loops containing regulated demographic parameters and at least one term in $Q$ that is affected by both a trait and a regulated demographic parameter for selection to be frequency-dependent. Given $\operatorname{dim} \mathscr{E}>1$, these requirements are fulfilled if two loops exist that are both evolving and regulated (Eq. 22). If no such two loops exist, more than two loops are required. With three loops the requirements are fulfilled if at least one of them is affected by both a trait and a regulated parameter (Eq. 23). If no such loop exists, then four loops are required of which at least one loop containing a trait and one loop containing a regulated demographic parameter have to be unconnected (Eq. 24). Adding more loops containing traits and/or regulated demographic parameters results in more summands of $Q$ to be affected by both traits and regulated demographic parameters and possibly in products of three or more loops that are affected by traits and/or regulated parameters but not in scenarios that cannot be attributed to one of the above three cases.

We emphasize that Proposition 17 only gives necessary but not sufficient conditions for selection to be frequency-dependent. The conditions are not sufficient because they do not exclude optimisation principles like those presented in Propositions 13, 15 and 16.

\section{Curvature of invasion boundaries}

\subsection{Invasion boundaries in trait spaces with two dimensions}

In trait space, invasion boundaries delimit sets of mutant trait vectors with positive invasion fitness from sets of mutant trait vectors with negative invasion fitness. The curvature of an invasion boundary at a singular point determines the set of trade-off curves that result in either an uninvadable or invadable singular point (cf. Sect. 2.5).

The curvature of invasion boundaries $\left\{\left(x_{1}, x_{2}\right) \mid x_{2}=I\left(x_{1}\right)\right\}$ at a singular point in a two-dimensional trait space can be determined by differentiating the equation $p\left(\left(x_{1}, I\left(x_{1}\right)\right), \hat{\boldsymbol{E}}\right)=0$ twice for $x_{1}$, where $p$ can be any fitness proxy. In the resulting equation we have to solve for $\mathrm{d}^{2} I / \mathrm{d} x_{1}^{2}$ and evaluate it at the singular point. We will use the following terminology. Loops that contain exactly one trait are called singletrait loops, loops that contain more than one trait are called multi-trait loops. We state sufficient conditions for invasion boundaries to be linear, convex or concave in the following result.

Proposition 18 (i) Invasion boundaries are linear if all evolving loops are single trait loops and all pairs of evolving loops are connected.

(ii) Invasion boundaries are convex if at least one multi-trait loop exists and all pairs of evolving loops are connected. 
Table 1 Curvature of invasion boundaries as a function of the presence or absence of unconnected evolving loops (columns) and the presence or absence of multi-trait loops (rows)

\begin{tabular}{lll}
\hline At least one multi-trait loop & At least one pair of unconnected evolving loops & \\
\cline { 2 - 3 } & Yes & No \\
\hline Yes & Parameter dependent & Convex \\
No & Concave & Linear \\
\hline
\end{tabular}

(iii) Invasion boundaries are concave if all evolving loops are single trait loops and at least one pair of evolving loops exists that is unconnected.

\section{Proof See Appendix D}

Note that these conditions are independent of the set of regulated demographic parameters and of the functional form of regulatory functions. From Eq. (D1) and (D2) in Appendix D it becomes clear that an increasing number of multi-trait loops increases the convexity of the invasion boundaries at the singular point while an increasing number of unconnected evolving loops increase the concavity. Whether invasion boundaries are convex or concave in life cycles with both multi-trait loops and unconnected evolving loops depends on the exact parameter values. These relationships are summarized in Table 1.

An intuitive understanding for these results can be gained from the simplest cases. Consider a life cycle with only one loop: $Q=-1+\tilde{f}_{12}^{\prime} \tilde{s}_{21}^{\prime}$. Invasion boundaries consist of all trait vectors $\left(s_{21}, f_{12}\right)$ with $0=-1+\tilde{f}_{12} \tilde{s}_{21}$, or, equivalently, with $\tilde{f}_{12}=1 / \tilde{s}_{21}$. Thus, invasion boundaries are convex. Alternatively, assume that additionally the two self-loops $\tilde{s}_{11}$ and $\tilde{s}_{22}$ exist and that these are evolving. Then $Q=$ $\tilde{f}_{12} \tilde{s}_{21}-\left(1-\tilde{s}_{11}^{\prime}\right)\left(1-\tilde{s}_{22}^{\prime}\right)$. Invasion boundaries consist of all trait vectors $\left(s_{11}, s_{22}\right)$ with $0=\tilde{f}_{12} \tilde{s}_{21}-\left(1-\tilde{s}_{11}\right)\left(1-\tilde{s}_{22}\right)$, or, equivalently, with $\tilde{s}_{22}=1-\tilde{f}_{12} \tilde{s}_{12} /\left(1-\tilde{s}_{11}\right)$. Thus, invasion boundaries are concave.

Based on these results we can draw the following general conclusions about the local invadability of singular points. Trade-offs between traits that affect the same loop correspond to convex invasion boundaries and are thus more likely to result in uninvadable singular points. Trade-offs between traits that affect unconnected loops correspond to concave invasion boundaries and are thus more likely to result in invadable singular points. These two scenarios are separated by trade-offs between traits that affect different but connected loops. These results are illustrated with examples in Sect. 7 and the corresponding Fig. 7.

\subsection{Invasion boundaries in trait spaces with more than two dimensions}

In this section we show that for models characterized by a high degree of symmetry Proposition 18 can be extended to trait spaces with more than two dimensions. In an $m$-dimensional trait space both the trade-off and the invasion boundary become $(m-1)$-dimensional manifolds which we characterize, without loss of generality, with 
$x_{m}=T\left(x_{1}, \ldots, x_{m-1}\right)$ and $x_{m}=I\left(x_{1}, \ldots, x_{m-1}\right)$, respectively. For a manifold to qualify as a trade-off manifold we require that at any point $x$ on the trade-off manifold the vectors orthogonal to the tangent plane of the manifold can all be chosen to point into the interior of the positive cone. In the representation of our choice this translates into $\partial T / \partial x_{j}<0$ for all $j \in\{1, \ldots, m-1\}$ (Appendix E). The invasion boundary is implicitly defined by

$$
\rho\left(\left(x_{1}, \ldots, x_{m-1}, I\left(x_{1}, \ldots, x_{m-1}\right)\right), \hat{\boldsymbol{E}}\right)=0
$$

where we may replace $\rho$ with any fitness proxy $p$. For figures of invasion boundaries and trade-off surfaces in three-dimensional spaces see Ravigné et al. (2009). As in two-dimensional trait spaces also in higher dimensional trait spaces both the trade-off manifold and the invasion boundaries divide the positive cone of $\mathbb{R}^{m}$ into an inward part adjacent to the origin, the feasibility set, and an outward part. For the invasion boundary, the outward part consists of all trait vectors with a positive invasion fitness and the inward part consists of all trait vectors with a negative invasion fitness given the resident community that determines the fitness landscape. From the same arguments as in Sect. 2.5 it follows that at a singular point the trade-off manifold and the invasion boundary are tangent to each other.

For two-dimensional trait spaces singular points are uninvadable if (i) the tradeoff is concave while the invasion boundary is either less strongly concave, linear or convex, or (ii) the trade off is linear and the invasion boundary is convex, or (iii) the trade-off is convex while the invasion boundary is more strongly convex. In the following we will extend these ideas to trait spaces with more than two dimensions. By definition, an invasion boundary is locally convex around a singular point $\boldsymbol{x}^{*}$ if and only if for any two points $z_{\mathbf{1}}, z_{2} \in B_{\epsilon}\left(x_{1}^{*}, \ldots, x_{m-1}^{*}\right)$

$$
\gamma I\left(z_{1}\right)+(1-\gamma) I\left(z_{2}\right)>I\left(\gamma z_{1}+(1-\gamma) z_{2}\right) \text { for } \gamma \in(0,1)
$$

and locally concave if and only if the opposite inequality holds true. In order to characterize uninvadability of singular points we define the following partial order $\stackrel{*}{\succ}$, pronounced as "is locally more convex than", on the set of manifolds of the same dimension that are tangent to each other at a singular point $\boldsymbol{x}^{*}$ :

$$
U \stackrel{*}{\succ} V: \Longleftrightarrow(\mathrm{H}(U)-\mathrm{H}(V))\left(x_{1}^{*}, \ldots, x_{m-1}^{*}\right) \text { positive definite, }
$$

where $\mathrm{H}(U)$ and $\mathrm{H}(V)$ denote the Hessian matrix of the functions $U\left(x_{1}, \ldots, x_{m-1}\right)$ and $V\left(x_{1}, \ldots, x_{m-1}\right)$, respectively. Note that this definition is independent of our choice of representation of the surfaces. In Appendix E we show that a singular point is uninvadable if and only if $I \stackrel{*}{\succ} T$. In particular this means that a singular point is uninvadable if the invasion boundary is a convex manifold while the trade-off manifold is linear or concave or if the invasion boundary is a linear manifold while the trade-off boundary is a concave manifold.

Proposition 18(i) characterizing sufficient conditions for linear invasion boundaries in two-dimensional trait spaces applies to higher dimensions without any restrictions. 
Proposition 19 Invasion boundaries are linear if all evolving loops are single-trait loops and if no pair of evolving loops exist that is unconnected.

\section{Proof See Appendix E}

Example Consider an age-structured population with $Q=-1+\sum_{k=1}^{n} L_{k}$, where $L_{k}=\tilde{f}_{1 k} \tilde{s}_{k(k-1)} \ldots \tilde{s}_{21}$. Assume that the fecundities at all age classes are evolving, $\boldsymbol{x}=\left(f_{11}, f_{12}, \ldots, f_{1 n}\right)$, and that each is traded off with all other fecundities. All loops pass through i-state 1, the newborns. With Proposition 19 we can conclude that invasion boundaries are linear. Thus, concave trade-off manifolds result in uninvadable singular points. If, however, invasion boundaries are convex in at least some direction, then singular points are invadable. The same conclusion can be drawn if the life cycle is size-structured instead of age-structured, i.e., if individuals can either stay in their i-state or move on to the next one.

The conditions for convex and concave invasion boundaries from Proposition 18(ii) and 18(iii) do not extend to trait spaces of arbitrary dimension. In fact, counterexamples can be constructed. However, under sufficient symmetry assumptions an extension of Proposition 18(ii) and 18(iii) becomes possible. We call a singular point $\boldsymbol{x}^{*}=$ $\left(x_{1}^{*}, \ldots, x_{m}^{*}\right)$ symmetric if

(a) $\left.\frac{\partial T}{\partial x_{1}}\right|_{*}=\cdots=\left.\frac{\partial T}{\partial x_{m-1}}\right|_{*}=-1$

(b) $Q\left(\boldsymbol{x}^{\prime}, \hat{\boldsymbol{E}}\left(\boldsymbol{x}^{*}\right)\right)=Q\left(\sigma\left(\boldsymbol{x}^{\prime}\right), \hat{\boldsymbol{E}}\left(\boldsymbol{x}^{*}\right)\right)$ for any permutation $\sigma$ of $\boldsymbol{x}^{\prime}$.

Our definition of symmetry is purely motivated by the requirements of the proofs. We expect that in most instances symmetry of a singular point will derive from some model symmetry as in the example that follows below.

Proposition 20 (i) Invasion boundaries at a symmetric singular point are convex if at least one multi-trait loops exists and all pairs of evolving loops are connected.

(ii) Invasion boundaries at a symmetric singular point are concave if all evolving loops are single-trait loops and at least one pair of evolving loops exist that is unconnected

\section{Proof See Appendix E.}

Remark 1 By continuity Proposition 20 also holds good if 'symmetric' is replaced with 'sufficiently close to symmetric'.

Example Consider a metapopulation occupying $n$ patches. Adults are sessile and survive from one season to the next with patch-specific probability $\tilde{s}_{k k}$. Adults reproduce and their newborns disperse over all patches such $\tilde{f}_{k l}$ newborns enter patch $k$ per adult occupying patch $l$. Assume that the patch-specific adult survival probabilities are evolving, $\boldsymbol{x}=\left(s_{11}, s_{22}, \ldots, s_{n n}\right)$, and that each is traded off with all other adult survival probabilities. Then all evolving loops are self-loops and therefore pairwise unconnected single trait loops. If the model is sufficiently symmetric such that 
(a)

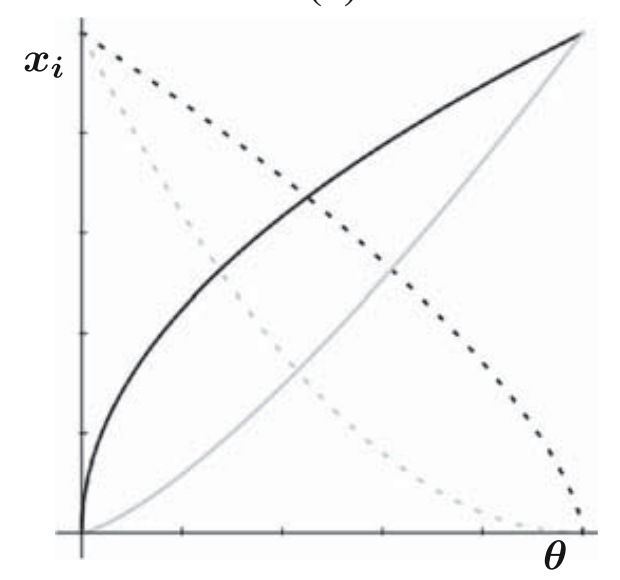

(b)

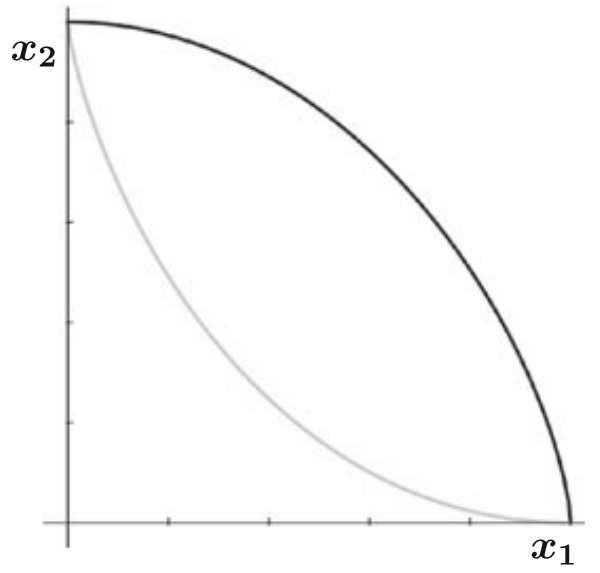

Fig. 6 Relationship between the allocation functions $x_{1}: \theta \mapsto x_{1}(\theta)$ and $x_{2}: \theta \mapsto x_{2}(\theta)$ and the trade-off curve $x_{2}=T\left(x_{1}\right)$. In a the allocation rule $x_{1}(\theta)=\theta^{1 / c_{1}}$ (solid curves) and $x_{2}(\theta)=(1-\theta)^{1 / c_{2}}$ (hatched curves) are plotted for two different sets of parameter values. Gray curves correspond to $c_{1}=0.75$ and $c_{2}=0.5$, giving rise to convex allocation functions. Black curves correspond to $c_{1}=2$ and $c_{2}=1.5$, giving rise to concave allocation functions. The resulting trade-off curve satisfying $x_{2}=T\left(x_{1}\right)=\left(1-x_{1}^{c_{1}}\right)^{1 / c_{2}}$ is plotted in (b). Two convex allocation functions give rise to a convex trade-off (gray curve) while two concave allocation functions give rise to a concave trade-off (black curve)

$\boldsymbol{x}^{*}=\left(s_{11}^{*}, s_{22}^{*}, \ldots, s_{n n}^{*}\right)$ is a symmetric singular point we can apply Proposition 20(ii). Then, at $\boldsymbol{x}^{*}$ the invasion boundary is concave.

\section{Frequency-dependent selection and linear invasion boundaries}

Under frequency-dependent selection attractivity is no longer coupled to uninvadability and has to be determined separately. In this case we can make detailed predictions only by restricting our model family.

In the following we restrict ourselves to two traits. Instead of describing the phenotypes on the trade-off curve with a two-dimensional vector $\left(x_{1}, x_{2}\right)$ we parameterize the trade-off with a scalar $\theta \in[0,1]: \boldsymbol{x}(\theta)=\left(x_{1}(\theta), x_{2}(\theta)\right)$. This notation can be motivated by interpreting $\theta$ as the fraction of a limited resource that is allocated to one of the traits while the remaining fraction $1-\theta$ is allocated to the other trait. We refer to the functions $x_{i}: \theta \mapsto x_{i}(\theta)$ as allocation functions and assume, without loss of generality, that $\mathrm{d} x_{1} / \mathrm{d} \theta>0$ and $\mathrm{d} x_{2} / \mathrm{d} \theta<0$. Furthermore, we assume that we can choose the parameterization with $\theta$ such that the curves given by $x_{1}(\theta)$ and $x_{2}(\theta)$ are either both convex or both concave: $\operatorname{sign}\left[\mathrm{d}^{2} x_{1} / \mathrm{d} \theta^{2}\right]=\operatorname{sign}\left[\mathrm{d}^{2} x_{2} / \mathrm{d} \theta^{2}\right]$. In Appendix $\mathrm{C}$ we prove that if both allocation functions $x_{i}(\theta)$ are convex (concave), then the trade-off curve $x_{2}=T\left(x_{1}\right)$ is also convex (concave) (cf. Fig. 6). More importantly for our purpose, also the converse is true: if the trade-off curve is convex (concave), then there exist two convex (concave) functions $\xi_{1}$ and $\xi_{2}$ with $\xi_{i}:[0,1] \rightarrow \mathbb{R}: \theta \mapsto x_{i}$.

Next we introduce some notation for the purpose of the following proposition. We denote regulatory functions that occur in loops containing $x_{1}$ as $R_{1}$ and regulatory functions that occur in loops containing $x_{2}$ as $R_{2}$. It will be helpful to omit the feedback environment that mediates the effect of the resident type on the mutant from our notation. We will therefore write $Q\left(\theta^{\prime}, \theta\right)$ instead of $Q\left(\theta^{\prime}, \hat{\boldsymbol{E}}(\theta)\right)$ and $R_{i}(\theta)$ instead 
of $R_{i}(\hat{E}(\theta))$. Furthermore, $\mathcal{L}_{x_{j} R_{j}}$ denotes the set of loops that contain both the trait $x_{j}$ and the regulatory function $R_{j}$ and $\mathcal{L}_{x_{j} \backslash R_{j}}:=\mathcal{L}_{x_{j}} \backslash \mathcal{L}_{x_{j} R_{j}}$ denotes the set of loops that contain $x_{j}$ but no regulatory function. We use $\left.\right|_{*}:=\left.\right|_{\theta^{\prime}=\theta=\theta^{*}}$ where $\theta^{*}$ is a singular point.

Proposition 21 Assume all loops containing a trait are pairwise connected single trait loops. Furthermore, all loops with a trait contain at most a single regulatory function and loops sharing the same trait contain the same regulatory function. Then

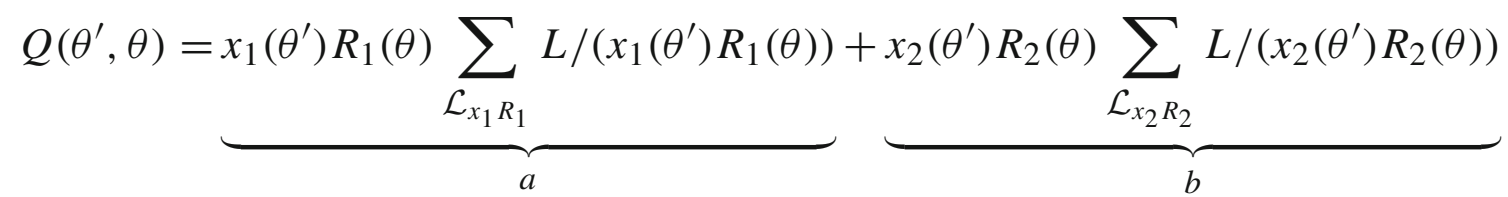

$$
\begin{aligned}
& +\underbrace{x_{1}\left(\theta^{\prime}\right) \sum_{\mathcal{L}_{x_{1} \backslash R_{1}}} L / x_{1}\left(\theta^{\prime}\right)}_{c}+\underbrace{x_{2}\left(\theta^{\prime}\right) \sum_{\mathcal{L}_{x_{2} \backslash R_{2}}} L / x_{1}\left(\theta^{\prime}\right)}_{d}+\operatorname{rest}(\boldsymbol{R}) .
\end{aligned}
$$

Either $a=0$ or $c=0$ and either $b=0$ or $d=0$ are allowed provided that not both $a=0$ and $b=0$.

(i) If $\left.\frac{\mathrm{d} R_{1}}{\mathrm{~d} \theta}\right|_{*}<0$ and $\left.\frac{\mathrm{d} R_{2}}{\mathrm{~d} \theta}\right|_{*}>0$, then a constant $k>0$ exists such that $\theta^{*}$ is a

- evolutionary repellor for $\mathrm{d}^{2} T\left(x_{1}\right) /\left.\mathrm{d} x_{1}^{2}\right|_{*}>k$.

- evolutionary branching point for $k>\mathrm{d}^{2} T\left(x_{1}\right) /\left.\mathrm{d} x_{1}^{2}\right|_{*}>0$.

- attracting and uninvadable for $\mathrm{d}^{2} T\left(x_{1}\right) /\left.\mathrm{d} x_{1}^{2}\right|_{*}<0$.

(ii) If $\left.\frac{\mathrm{d} R_{1}}{\mathrm{~d} \theta}\right|_{*}>0$ and $\left.\frac{\mathrm{d} R_{2}}{\mathrm{~d} \theta}\right|_{*}<0$, then a constant $k<0$ exists such that $\theta^{*}$ is a

- evolutionary repellor for $\mathrm{d}^{2} T\left(x_{1}\right) /\left.\mathrm{d} x_{1}^{2}\right|_{*}>0$.

- Garden of Eden-point for $0>\mathrm{d}^{2} T\left(x_{1}\right) /\left.\mathrm{d} x_{1}^{2}\right|_{*}>k$.

- attracting and uninvadable for $\mathrm{d}^{2} T\left(x_{1}\right) /\left.\mathrm{d} x_{1}^{2}\right|_{*}<k$.

If $a=0(b=0)$, then the condition on the sign of $\mathrm{d} R_{1} /\left.\mathrm{d} \theta\right|_{*}\left(\mathrm{~d} R_{2} /\left.\mathrm{d} \theta\right|_{*}\right)$ can be dropped.

Proof Since evolving loops are single trait loops and pairwise connected it follows from Proposition 18(i) that invasion boundaries are linear. Thus, convex trade-offs $\left(\mathrm{d}^{2} T\left(x_{1}\right) /\left.\mathrm{d} x_{1}^{2}\right|_{*}>0\right)$ correspond to invadable and concave trade-offs $\left(\mathrm{d}^{2} T\left(x_{1}\right) /\left.\mathrm{d} x_{1}^{2}\right|_{*}\right.$ $<0)$ to uninvadable singular points. The condition for attractivity in one-dimensional trait spaces is

$$
0>\partial^{2} \rho\left(\theta^{\prime}, \theta\right) /\left.\partial \theta^{\prime 2}\right|_{*}+\partial^{2} \rho\left(\theta^{\prime}, \theta\right) /\left.\partial \theta^{\prime} \partial \theta\right|_{*}
$$


(Metz et al. 1996b; Geritz et al. 1998), where invasion fitness $\rho$ might be replaced with any fitness proxy $p$. Applying this criterion to $Q\left(\theta^{\prime}, \theta\right)$ yields

$$
\begin{aligned}
& 0>\left(\frac{\mathrm{d}^{2} x_{1}}{\mathrm{~d} \theta^{\prime 2}} R_{1}(\theta) \sum_{\mathcal{L}_{x_{1}^{\prime} R_{1}}} L /\left(x_{1}\left(\theta^{\prime}\right) R_{1}(\theta)\right)+\frac{\mathrm{d}^{2} x_{2}}{\mathrm{~d} \theta^{\prime 2}} R_{2}(\theta) \sum_{\mathcal{L}_{x_{2}^{\prime} R_{2}}} L /\left(x_{2}\left(\theta^{\prime}\right) R_{2}(\theta)\right)\right.
\end{aligned}
$$

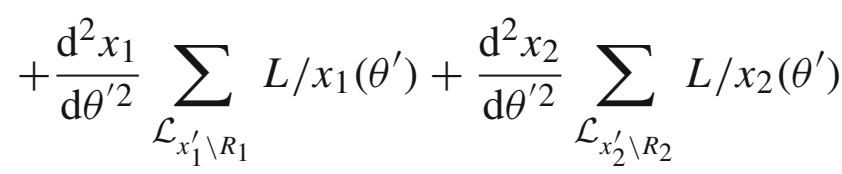

$$
\begin{aligned}
& \left.+\frac{\mathrm{d} x_{1}}{\mathrm{~d} \theta^{\prime}} \frac{\mathrm{d} R_{1}}{\mathrm{~d} \theta} \sum_{\mathcal{L}_{x_{1}^{\prime} R_{1}}} L /\left(x_{1}\left(\theta^{\prime}\right) R_{1}(\theta)\right)+\frac{\mathrm{d} x_{2}}{\mathrm{~d} \theta^{\prime}} \frac{\mathrm{d} R_{2}}{\mathrm{~d} \theta} \sum_{\mathcal{L}_{x_{2}^{\prime} R_{2}}} L /\left(x_{2}\left(\theta^{\prime}\right) R_{2}(\theta)\right)\right)\left.\right|_{*} .
\end{aligned}
$$

All double derivatives have the same sign. They are positive for convex trade-offs and negative for concave ones. The products of derivatives in the last two terms of Condition (27) are negative under the assumptions in (i) and positive under the assumptions in (ii). We start with case (i). Then, with concave trade-offs, all terms on the righthand side of Condition (27) are negative and the condition is fulfilled. For the case of a linear trade-off the double derivatives equal zero and the condition for attractivity is still fulfilled. Changing the curvature of the trade-off slightly towards convex will generically not change the sign of the right-hand side of Condition (27) and therefore for sufficiently weakly convex trade-offs the condition is still fulfilled. Only for sufficiently convex trade-off curves where the sum of the first four terms exceeds the sum of the last two terms is Condition 27 not fulfilled. The proof for case (ii) is obtained by exchanging the words concave and convex, positive and negative, fulfilled and not fulfilled.

An application of Proposition 21 can be found in Sect. 7. Case (i) corresponds to the common situation that a high transmission through one loop is accompanied by strong density-dependent regulation of that loop because many individuals compete for the limiting resources used while passing through the i-states of the loop. For example, increasing fecundity at the cost of decreased adult survival results in more newborn individuals and in fewer adult individuals and thereby intensifies competition between juveniles and lessens competition between adults. This pattern corresponds to negative frequency dependence: in a population where the majority of individuals are long-lived at the cost of low fecundity a rare type with high fecundity benefits because the fecundity loop experiences only weak density dependence. This advantage persists as long as the mutant remains sufficiently rare and does not contribute or only slightly contributes to population regulation. It is this temporary advantage that allows a mutant to invade. This mechanism favors evolution towards phenotypes that accrue fitness via different loops, i.e., phenotypes with intermediate values of $\theta$. Case (ii) corresponds to the rather counterintuitive reversed situation of positive frequency dependence. In this case loops with high transmission are affected relatively little by density dependence which favors evolution towards extreme phenotypes that accrue fitness only via few loops. With positive frequency dependence, evolution towards an 
intermediate phenotype is only possible for sufficiently concave trade-offs that offset the effect of positive frequency dependence.

\section{Examples: evolution in a stage-structured population}

In this section we return to the questions raised in the introduction. How robust are the results by Takada and Nakajima (1996) with respect to assumptions about the choice of traits and the precise action of population regulation? The purpose of this section is to show how our toolbox simplifies the analysis and allows for a priori qualitative predictions. We will apply our results to various models based on the life cycle graph in Fig. 2. This life cycle corresponds to a hypothetical perennial plant with a seedling, juvenile and flowering stage. Seedlings turn into juveniles with probability $\tilde{s}_{21}$ and juveniles turn into flowering plants with probability $\tilde{s}_{32}$. Juvenile and flowering plants persist in their current i-state with probability $\tilde{s}_{22}$ and $\tilde{s}_{33}$, respectively. Flowering plants reproduce via seeds, resulting in $\tilde{f}_{13}$ seedlings, or via vegetative propagules, resulting in $\tilde{f}_{23}$ juvenile plants in the next season.

Takada and Nakajima $(1992,1996)$ analyzed three versions of their model that differ in the set of traits and regulated demographic parameters. We introduce the different models by giving the corresponding fitness proxy $Q$ in which the assumptions of each variant are made explicit:

$$
\begin{aligned}
Q\left(\left(f_{13}^{\prime}, s_{33}^{\prime}\right), \hat{\boldsymbol{E}}\right)= & f_{13}^{\prime} R_{\mathrm{f}, 13}(\hat{\boldsymbol{E}}) s_{32} s_{21}+f_{23} s_{32}-\left(1-s_{22}\right)\left(1-s_{33}^{\prime}\right) \\
Q\left(\left(f_{13}^{\prime}, f_{23}^{\prime}\right), \hat{\boldsymbol{E}}\right)= & f_{13}^{\prime} R_{\mathrm{f}, 13}(\hat{\boldsymbol{E}}) s_{32} s_{21} R_{\mathrm{s}, 21}(\hat{\boldsymbol{E}})+f_{23}^{\prime} s_{32} \\
& -\left(1-s_{22}\right)\left(1-s_{33}\right) \\
Q\left(\left(f_{13}^{\prime}, f_{23}^{\prime}\right), \hat{\boldsymbol{E}}\right)= & f_{13}^{\prime} s_{32} R_{\mathrm{s}, 32}(\hat{\boldsymbol{E}}) s_{21}+f_{23}^{\prime} s_{32} R_{\mathrm{s}, 32}(\hat{\boldsymbol{E}}) \\
& -\left(1-s_{22} R_{\mathrm{s}, 22}(\hat{\boldsymbol{E}})\right)\left(1-s_{33} R_{\mathrm{s}, 33}(\hat{\boldsymbol{E}})\right)
\end{aligned}
$$

In all three cases the traits affect different loops that start at the flowering plant-state. Thus, in the terminology of Sect. 2.5, the evolving loops are pairwise connected single trait loops. From Proposition 18 follows that invasion boundaries are linear and singular points on a convex trade-off are invadable while singular points on a concave trade-off are uninvadable.

As shown by Takada and Nakajima (1992, 1996), all three models can be analyzed by investigating an optimisation principle that does not require to calculate $\hat{\boldsymbol{E}}$. By choosing $\mathcal{M}=\left\{\tilde{f}_{13} \tilde{s}_{32} \tilde{s}_{21}\right\}$, Eq. (28) and (29) follow the pattern of 1(e) in Sect. 3.2 and $\prod_{\breve{x}_{\mathcal{M}}} x_{j} /|b(\boldsymbol{x})|=f_{13} /\left|f_{23} s_{32}-\left(1-s_{22}\right)\left(1-s_{33}\right)\right|$ is an optimisation principle. Similarly, by choosing $\mathcal{M}=\left\{\tilde{f}_{13} \tilde{s}_{32} \tilde{s}_{21}, \tilde{f}_{23} \tilde{s}_{32}\right\}$ Eq. (30) follows the pattern of 1(f) in Sect. 3.2 and $\sum_{\mathcal{M}}[L / R](\boldsymbol{x})=f_{13} s_{32} s_{21}+f_{23} s_{32}$ is an optimisation principle.

Takada and Nakajima assume that $\hat{\boldsymbol{E}}=\sum_{i=1}^{3} \hat{N}_{i}$ and therefore, as stated by the authors and as can be concluded from Corollary $14, \hat{\boldsymbol{E}}$ is an equivalent optimisation principle. However, the derivation of the above optimisation principles does not involve $\hat{\boldsymbol{E}}$ and therefore no assumptions about the regulatory functions are actually required. 
In the remainder of this section we analyze three variants of the model by Takada and Nakajima $(1992,1996)$. These variants differ from the ones above in that the existence of an optimisation principle does depend on the precise nature of $\hat{\boldsymbol{E}}$. In these examples selection becomes frequency-dependent whenever $\operatorname{dim} \mathscr{E}>1$ because the corresponding fitness proxy $Q$ follows the pattern of Eq. (22). To showcase the full spectrum of possibilities we have constructed the examples such that we will encounter convex, linear and concave invasion boundaries.

We start with the assumption of Takada and Nakajima $(1992,1996)$ that the production of seeds and vegetative propagules is traded off (cf. Eqs. (29) and (30)). Thus, invasion boundaries are linear. In contrast to the examples above we now assume that both the number of surviving seeds and vegetative propagules are regulated:

$$
Q\left(\left(f_{13}^{\prime}, f_{23}^{\prime}\right), \hat{\boldsymbol{E}}\right)=f_{13}^{\prime} R_{\mathrm{f}, 13}(\hat{\boldsymbol{E}})_{32} s_{21}+f_{23}^{\prime} R_{\mathrm{f}, 23}(\hat{\boldsymbol{E}}) s_{32}-\left(1-s_{22}\right)\left(1-s_{33}\right)
$$

If the effective fecundity through both modes is limited by the same resources such that $R_{\mathrm{f}, 13}(\hat{\boldsymbol{E}})=R_{\mathrm{f}, 23}(\hat{\boldsymbol{E}})$, then Eq. (31) follows the pattern of 1(f) in Sect. 3.2. To see this we have to choose $\left.\mathcal{M}=\left\{\tilde{f}_{13} \tilde{s}_{32} \tilde{s}_{21}, \tilde{f}_{23} \tilde{s}_{32}\right)\right\}$ and $\sum_{\mathcal{M}}\left[L / \boldsymbol{R}_{L}\right](\boldsymbol{x})=$ $f_{13} s_{32} s_{21}+f_{23} s_{32}$ will be maximized. If the trade-off is convex, singular points are evolutionary repellors and in case of concave trade-offs singular points are attracting and uninvadable and therefore endpoints of evolution. Figure 7 a shows a numerically calculated bifurcation diagram confirming these predictions. We note that the condition $R_{\mathrm{f}, 13}(\hat{\boldsymbol{E}})=R_{\mathrm{f}, 23}(\hat{\boldsymbol{E}})$ is not necessary for optimisation to be possible. It suffices that $\hat{\boldsymbol{E}}$ is a scalar and that $R_{\mathrm{f}, 13}(\hat{\boldsymbol{E}})$ and $R_{\mathrm{f}, 23}(\hat{\boldsymbol{E}})$ are both monotonically increasing (decreasing) in $\hat{\boldsymbol{E}}$ in which case we can conclude with Corollary 14 that $\hat{\boldsymbol{E}}(-\hat{\boldsymbol{E}})$ is an optimisation principle.

Next we consider the case that the number of seeds and vegetative propagules affect the survival of seeds and vegetative propagules, respectively. More precisely, $\hat{\boldsymbol{E}}=\left(f_{13} \hat{N}_{3}, f_{23} \hat{N}_{3}\right)$ with $R_{\mathrm{f}, 13}$ only varying in $\hat{E}_{1}$ and $R_{\mathrm{f}, 23}$ only varying in $\hat{E}_{2}$. Now selection is frequency-dependent. For Proposition 21 to be applicable it is required that at a singular point $\mathrm{d} R_{\mathrm{f}, 13}\left(f_{13}(\theta) \hat{N}_{3}(\theta)\right) / \mathrm{d} \theta \geq 0$ and $\mathrm{d} R_{\mathrm{f}, 23}\left(f_{23}(\theta) \hat{N}_{3}(\theta)\right) / \mathrm{d} \theta \leq 0$. If we parameterize the trade-off such that $\mathrm{d} f_{13}(\theta) / \mathrm{d} \theta>0$ and $\mathrm{d} f_{23}(\theta) / \mathrm{d} \theta<0$, this will be true if $\mathrm{d} \hat{N}_{3}(\theta) / \mathrm{d} \theta$ is sufficiently small. Figure $7 \mathrm{~b}$ shows a numerically calculated bifurcation diagram confirming the predictions from Proposition 21.

We will now modify the life cycle considered so far by assuming that juvenile plants are also capable of reproduction. Each individual produces $\tilde{f}_{12}$ seeds that enter the seedling stage in the next season. We will consider two different versions of this modified model. In the first one, $\tilde{s}_{21}$ and $\tilde{s}_{32}$ are evolving and $\tilde{s}_{21}$ and $\tilde{f}_{23}$ are under population regulation:

$$
\begin{aligned}
Q\left(\left(s_{21}^{\prime}, s_{32}^{\prime}\right), \hat{\boldsymbol{E}}\right)= & f_{12} s_{21}^{\prime} R_{\mathrm{s}, 21}(\hat{\boldsymbol{E}})\left(1-s_{33}\right)+f_{13} s_{32}^{\prime} s_{21}^{\prime} R_{\mathrm{s}, 21}(\hat{\boldsymbol{E}}) \\
& +f_{23} R_{\mathrm{f}, 23}(\hat{\boldsymbol{E}}) s_{32}^{\prime}-\left(1-s_{22}\right)\left(1-s_{33}\right)
\end{aligned}
$$

In this model evolution affects three connected loops, one of which is a multi-trait loop. Hence, according to Proposition 18 invasion boundaries are convex and only singular 

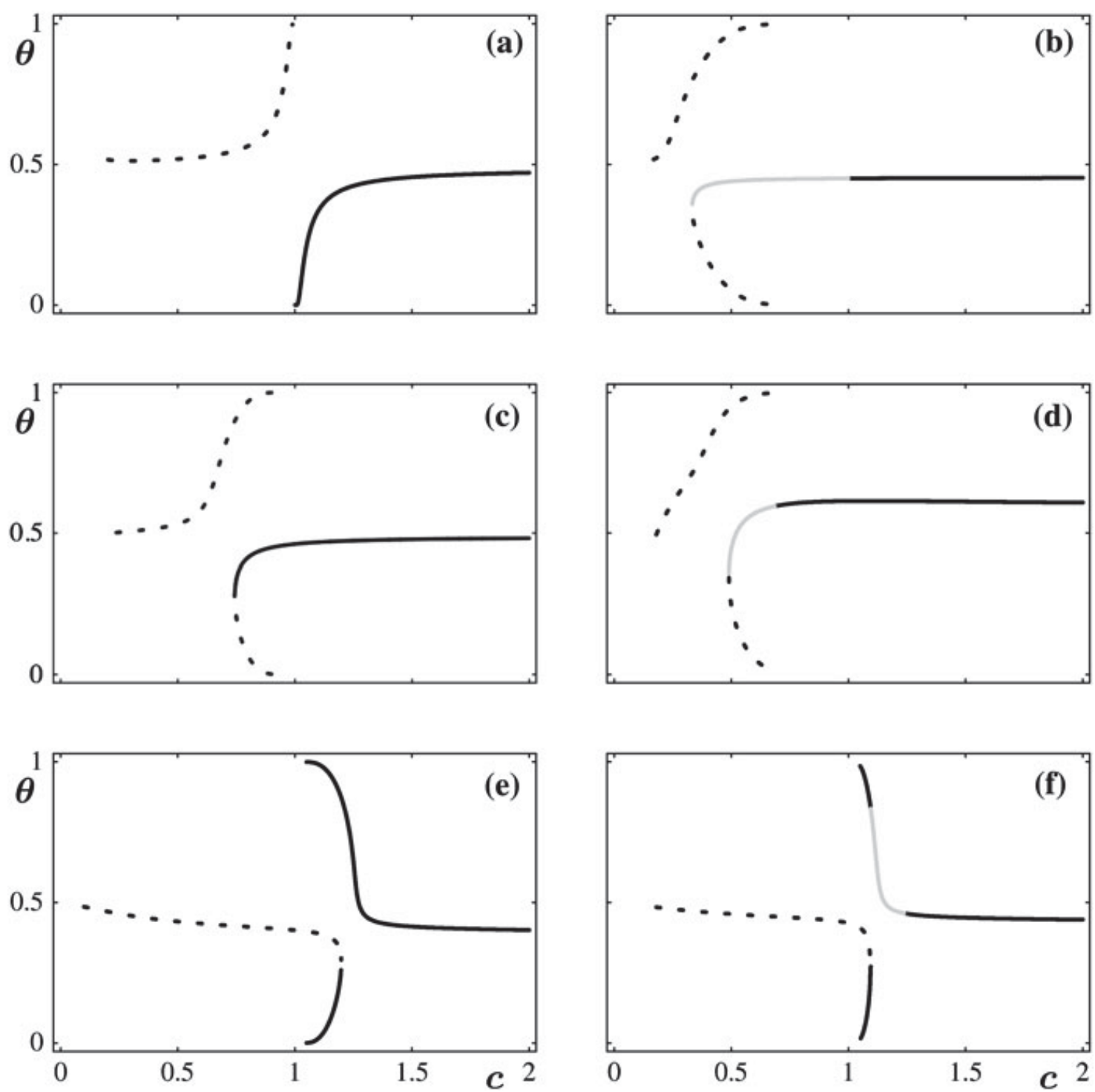

Fig. 7 Bifurcation of singular points $\theta^{*}$ (on $y$-axis) as a function of the curvature of the trade-off (on $x$-axis). Panels a, b correspond to Eq. (31), c, d to Eq. (32) and e, f to Eq. (33). The left column shows results for the frequency-independent and the right column for the frequency-dependent version of the respective model. The trade-off curve is parameterized by $\left(x_{1}(\theta), x_{2}(\theta)\right)=\left(x_{\max } \theta^{1 / c}, x_{\max }(1-\theta)^{1 / c}\right)$. Here $c$ determines the curvature of the trade-off such that it is convex for $c<1$, linear for $c=1$ and concave for $c>1$. Solid black lines correspond to singular points that are attracting and uninvadable, hatched black lines to evolutionary repellors and solid grey lines to evolutionary branching points. Parameter values are chosen for optimal presentation of the characteristic structure of the bifurcation diagram. $\mathbf{a}, \mathbf{b} \boldsymbol{x}=\left(f_{13}, f_{23}\right), f_{13 \max }=100, f_{23 \max }=20, s_{21}=0.3, s_{22}=0.3, s_{32}=0.5, s_{33}=0.3, \alpha=0.1$. $\mathbf{a} \beta=0.07, R_{\mathrm{f}, 13}=1 /\left(1+\alpha N_{3}\right), R_{\mathrm{f}, 23}=1 /\left(1+\beta N_{3}\right) . \mathbf{b} \beta=0.4, R_{\mathrm{f}, 13}=1 /\left(1+\alpha f_{13} N_{3}\right), R_{\mathrm{f}, 23}=$ $1 /\left(1+\beta f_{23} N_{3}\right)$. c, $\mathbf{d} \boldsymbol{x}=\left(s_{21}, s_{32}\right), f_{12}=50, f_{13}=100, f_{23}=20, s_{21 \max }=0.3, s_{22}=0.4$, $s_{32 \max }=0.4, s_{33}=0.4, \alpha=0.1$. c $\beta=0.08, R_{\mathrm{s}, 21}=1 /\left(1+\alpha N_{2}\right), R_{\mathrm{f}, 23}=1 /\left(1+\beta N_{2}\right)$. d $\beta=0.5, R_{\mathrm{s}, 21}=1 /\left(1+\alpha N_{2}\right), R_{\mathrm{f}, 23}=1 /\left(1+\beta N_{3}\right)$. e, f $\boldsymbol{x}=\left(s_{21}, s_{33}\right), f_{12}=40$, $f_{13}=10, f_{23}=28, s_{21 \max }=0.3, s_{22}=0.4, s_{32}=0.4, s_{33 \max }=0.4, \alpha=0.1$. e $\beta=0.1, R_{\mathrm{f}, 12}=$ $1 /\left(1+\alpha\left(N_{1}+N_{2}\right), R_{\mathrm{f}, 13}=1 /\left(1+\beta\left(N_{1}+N_{2}\right)=R_{\mathrm{f}, 23} . \mathbf{f} \beta=0.058, R_{\mathrm{f}, 12}=1 /\left(1+\alpha N_{2}\right), R_{\mathrm{f}, 13}=\right.\right.$ $1 /\left(1+\beta N_{3}\right)=R_{\mathrm{f}, 23}$

points on sufficiently strongly convex trade-offs are invadable. Figure $7 \mathrm{c}$ and d shows that indeed the change from an invadable to an uninvadable singular point occurs for a convex trade-off. If both $s_{21}$ and $f_{23}$ are decreasing functions of the density $N_{2}$ we can conclude with Proposition 13 that evolution will favor the trait combination that maxi- 
mizes $\hat{N}_{2}$. An optimisation principle based on traits can only be found when all regulatory functions are identical such that they can be factored out from the first three terms on the right hand side of Eq. (32). Then, by choosing $\mathcal{M}=\left\{\tilde{f}_{12} \tilde{s}_{21}, \tilde{f}_{13} \tilde{s}_{32} \tilde{s}_{21}, \tilde{f}_{23} \tilde{s}_{32}\right\}$, Eq. (32) follows the pattern 2(d) in Sect. 3.2 and $\sum_{\mathcal{M}}\left[L / \boldsymbol{R}_{L}\right](\boldsymbol{x}) q(\breve{L})(\boldsymbol{x}) /|b(\boldsymbol{x})|=$ $\left(f_{12} s_{21}\left(1-s_{33}\right)+f_{13} s_{32} s_{21}+f_{23} s_{32}\right) /\left(1-s_{22}\right)\left(1-s_{33}\right)$ is maximized. Since the denominator is constant it can actually be dropped.

Selection becomes frequency-dependent when $\tilde{s}_{21}$ and $\tilde{f}_{23}$ decrease with $N_{2}$ and $N_{3}$, respectively. Proposition 21 cannot be applied because of the presence of a multi-trait loop. However, branching points are still possible but can only occur when the tradeoff is sufficiently strongly convex. These predictions are confirmed by a numerically calculated bifurcation diagram (Fig. 7d).

In the second example based on the modified life cycle, $\boldsymbol{x}=\left(s_{21}, s_{33}\right)$ and all fertility parameters are under population regulation:

$$
\begin{aligned}
Q\left(\left(s_{21}^{\prime}, s_{33}^{\prime}\right), \hat{\boldsymbol{E}}\right)= & f_{12} R_{\mathrm{f}, 12}(\hat{\boldsymbol{E}}) s_{21}^{\prime}\left(1-s_{33}^{\prime}\right)+f_{13} R_{\mathrm{f}, 13}(\hat{\boldsymbol{E}}) s_{32} s_{21}^{\prime} \\
& +f_{23} R_{\mathrm{f}, 23}(\hat{\boldsymbol{E}}) s_{32}-\left(1-s_{22}\right)\left(1-s_{33}^{\prime}\right) .
\end{aligned}
$$

Evolution occurs in three single-trait loops, two of which are unconnected. Thus, according to Proposition 18 invasion boundaries are concave and singular points on convex, linear and weakly concave trade-offs are invadable and only singular points on sufficiently strongly concave trade-offs are uninvadable (Fig. 7e, f). Optimisation is possible if $\hat{\boldsymbol{E}}$ is a scalar in which case Corollary 14 can be applied, or if all regulatory functions are equal such that they can be factored out from the first three term on the right-hand side of Eq. (33). Then, by choosing $\mathcal{M}=\left\{\tilde{f}_{12} \tilde{s}_{21}, \tilde{f}_{13} \tilde{s}_{32} \tilde{s}_{21}, \tilde{f}_{23} \tilde{s}_{32}\right\}$ the fitness proxy $Q$ follows the pattern of $2(\mathrm{~d})$ in Sect. 3.2 and $\sum_{\mathcal{M}}\left[L / \boldsymbol{R}_{L}\right](\boldsymbol{x}) q(\breve{L})(\boldsymbol{x}) /$ $|b(\boldsymbol{x})|=\left(f_{12} s_{21}\left(1-s_{33}\right)+f_{13} s_{32} s_{21}+f_{23} s_{32}\right) /\left(1-s_{22}\right)\left(1-s_{33}\right)$ is maximized.

If $\tilde{f}_{12}$ decreases with $N_{2}$ while $\tilde{f}_{13}$ and $\tilde{f}_{23}$ decrease with $N_{3}$, then selection is frequency-dependent. Proposition 21 cannot be applied because of two unconnected loops. Branching points are still possible but can only occur for trade-offs of intermediate concavity. These predictions are confirmed by a numerically calculated bifurcation diagram (Fig. 7f).

\section{Discussion}

The purpose of this article is twofold. First, we aim at identifying key features that underly the evolution of demographic parameters in populations that are structured according to a finite number of states. Knowing such key features allows one to judge whether predictions derived from specific models are robust with respect to the assumed nature of population regulation and the chosen set of traits. Most importantly, having identified the exact conditions corresponding to a specific phenomenon is an important step towards a deep understanding of the phenomenon. Specifically, we (i) identify sufficient conditions in terms of the algebraic structure of the fitness function for the existence of optimisation principles (Sect. 3), (ii) give necessary conditions for selection to be frequency-dependent (Sect. 4), (iii) give simple conditions in terms of features of the life cycle that allow to determine whether or not singu- 
lar points are invadable (Sect. 5) and (iv) give sufficient conditions for models with frequency-dependent selection that allow to predict qualitatively how properties of singular points change as a function of the curvature of the trade-off (Sect. 6). Second, for a large group of models we give exact recipes to choose the correct quantity for a maximization procedure (Sects. 3.2 and 3.3). This should benefit researchers who are dealing with explicit models that fall within our model family. However, from the rather intricate prerequisites for the existence of an optimisation principle one might conclude that life cycles where evolutionary questions can be answered by maximizing an optimisation principle are the exception rather than the rule.

Our treatment is based on a fitness proxy $Q$ that is a simple function of the loop transmissions in a life cycle graph. A loop is defined as a sequence of demographic parameters that lead from one i-state to itself without passing through any i-state more than once and the corresponding loop transmission equals the product of the demographic parameters along the loop. $Q$ is the sum of all loop transmissions and of the products of loop transmissions of mutually unconnected loops where the sign of each term is positive if an uneven number of loop transmissions is multiplied and negative otherwise (cf. Eq. 5). The advantage of this fitness proxy is twofold. First, $Q$ is a linear function of all demographic parameters making it a good starting point for analytical investigations. Second, many of our results can be formulated in terms of properties of loops and are thus also readily interpreted in biological terms.

In our model we split the set of demographic parameters characterizing a life cycle into two groups, those that are constant and those that contain evolving traits. This division will in most cases be artificial. One can expect that for any demographic parameter mutations exist affecting it and selection will be acting on each of the demographic parameters. Such divisions are nevertheless useful and commonly applied. It often happens that models are re-analysed with a different set of parameters evolving or a different coupling by trade-offs. Our results allow to distinguish subsets of demographic parameters that evolve so as to maximize an optimisation principle from subsets that experience frequency-dependent selection. The identification of such subsets might also facilitate the analysis of models with many evolving traits.

Several important aspects of our work are incomplete. Most importantly, when the evolutionary dynamics is frequency-dependent we can only make predictions for models with linear invasion boundaries and rather simple modes of population regulation. It would be desirable to achieve a classification with respect to attractivity for a broader class of models. An approach that could in principle be useful in achieving such a classification has been introduced by Bowers et al. (2005). These authors show that attractivity can be derived from the curvature of the invasion boundary and a second curve, implicitly defined by $0=Q\left(\boldsymbol{x}^{*}, \hat{\boldsymbol{E}}(\boldsymbol{x})\right)$. This latter curve consists of all trait combinations $\left(x_{1}, x_{2}\right)$ for which, if these would characterize the resident type, a mutant with the singular trait combination $\boldsymbol{x}^{*}$ is selectively neutral. It divides the positive cone of $\mathbb{R}^{2}$ in trait combinations that, if common, either could or could not be invaded by the singular strategy. Deriving an explicit expression for the curvature of this curve requires an explicit expression for the second derivative of $Q\left(\boldsymbol{x}^{*}, \hat{\boldsymbol{E}}(\boldsymbol{x})\right)$ with respect to the trait value of the resident. This will generally only be possible if an explicit expression for the environment at population dynamical equilibrium $\hat{\boldsymbol{E}}$ exists. In our model class this is only fulfilled in the simplest cases and even then the 
expressions quickly become lengthy such that they can only be handled by means of programs capable of symbolic computation. The situation seems a bit better in continuous time models where Bowers (2010) derived for a family of Lotka-Volterra models results that are in the same spirit as those presented by us.

In Propositions 10-16 we present sufficient conditions for the existence of an optimisation principle. Conversely, in Proposition 17 we state necessary conditions for selection to be frequency-dependent such that optimisation is impossible. It is currently an open problem whether further constellations exist that allow for optimisation and what additional assumptions are necessary to turn Proposition 17 into a statement about necessary and sufficient conditions for frequency dependence.

Furthermore, in our framework we assumed that the regulatory functions are not subject to evolutionary change. However, selection is expected to act to diminish the sensitivity of organisms to the detrimental effects due to their conspecifics. Biologically, this could for example be realized by increasing the efficiency with which energy is extracted from resources. Mathematically, this corresponds to a slower decrease of the regulatory functions $R_{\mathrm{z}, l k}$ with increasing $\hat{E}_{i}(\mathcal{X})$. For instance, in case of the Beverton-Holt functional form $R_{\mathrm{z}, l k}(\hat{\boldsymbol{E}})=1 /\left(1+\alpha \sum \hat{N}_{i}(\boldsymbol{\mathcal { X }})\right)$, selection is expected to decrease the value of the constant $\alpha$. A more complete theory should allow for changes in the regulatory functions $R_{\mathrm{z}, l k}$ and for trade-offs between parameters affecting $R_{\mathrm{z}, l k}$ and demographic parameters.

Last but not least, in the case of trait spaces with more than two dimensions we can generally determine the curvature of invasion boundaries only for highly symmetric models. It might be feasible to prove statements with weaker symmetry assumptions than those employed by us if the class of models is restricted in appropriate ways.

Despite these limitations, our framework allows us to make several predictions about large-scale patterns in life history evolution and these predictions are what we consider our most important results. First, if the $n$ traits enter $Q\left(\boldsymbol{x}^{\prime}, \hat{\boldsymbol{E}}\right)$ as a product $x_{1}^{\prime} x_{2}^{\prime} \ldots x_{n}^{\prime}$, because the traits occur in a single loop or because all occur in a common set of loops, then selection results in a single optimal life history maximizing the product of traits. In this case, frequency dependence cannot occur and phenotypic variation is selected against. Thus, we predict that species that only differ in traits that affect the same loop cannot coexist. Second, if evolving traits affect unconnected loops, then invasion boundaries are concave and singular points are invadable, hence, either evolutionary repellors or evolutionary branching points, for a wide range of trade-off curvatures. In the case of an evolutionary repellor, selection favors increased loop transmission in one set of loops at the cost of decreased loop transmission in another set of loops. Ultimately, this can result in the complete shutdown of loops. Similarly, if evolutionary branching results in two diverging species, these increase the transmission rate in alternative sets of loops at the cost of a decreased transmission rate in the respective other set of loops. Taken together, we therefore predict that relatively few organisms have life cycles where unconnected loops exist next to each other. If they do, this might be due to a lack of traits within these loops that are traded-off with each other. Third, phenotypic diversification driven by negative frequency-dependent selection has to involve differences in the transmission rates of alternative loops, each of which associated with different limiting factors (cf. Proposition 17). 
Acknowledgments Work on this project spanned a long time during which C.R. received financial supported by the Netherlands Organisation of Scientific Research (NWO), a Discovery Grant to Peter Abrams from the Natural Sciences and Engineering Research Council of Canada and by the Vienna Science and Technology Fund (WWTF) through project MA07-015. H.M. was supported by the European Training Network ModLife funded through the Human Potential Program of the European Commission and T.V.D. was supported by a Dutch NWO-VENI grant. The authors thank Odo Diekmann, Mats Gyllenberg, Joachim Hermisson, Géza Meszéna and two anonymous referees for helpful comments.

\section{Appendix A: The fitness proxy $Q$}

Let $\mathrm{A}\left(\boldsymbol{x}^{\prime}, \hat{\boldsymbol{E}}\right)=\left[\tilde{a}_{k l}\right]$ be the $n \times n$ projection matrix corresponding to a given life cycle graph. Its dominant eigenvalue $\lambda_{\mathrm{d}}$ is the largest root that fulfills the characteristic equation $P\left(\lambda, \boldsymbol{x}^{\prime}, \hat{\boldsymbol{E}}\right)=\operatorname{det}(\lambda \mathrm{I}-\mathrm{A})=0$. Hubbell and Werner (1979) and Caswell (1982) showed that the characteristic polynomial can be written as

$$
\begin{aligned}
P\left(\lambda, \boldsymbol{x}^{\prime}, \hat{\boldsymbol{E}}\right)= & 1-\sum_{\mathcal{L}_{\mathrm{A}}} L \lambda^{-|\breve{L}|}+\sum_{\mathcal{L}_{\mathrm{A}}^{2 *}} L M \lambda^{-(|\breve{L}|+|\breve{M}|)} \\
& -\sum_{\mathcal{L}_{\mathrm{A}}^{3 *}} L M N \lambda^{-(|\breve{L}|+|\breve{M}|+|\breve{N}|)}+\cdots
\end{aligned}
$$

where $\mathcal{L}_{\mathrm{A}}$ denotes the set of all loops present in the life cycle graph corresponding to A, the summations marked with an asterisk run over all pairs, triplets, etc. of mutually unconnected loops and $|\breve{L}|$ denotes the number of i-states that belong to loop $L$. Evaluating the characteristic polynomial at $\lambda=1$ and multiplying it by -1 results in the fitness proxy $Q$ given by Eq. (5).

Under certain conditions the fitness proxy $Q$ can be transformed into the basic reproduction ratio $R_{0}$. This is possible if the life cycle under consideration has only a single birth state, say i-state 1 . We collect all loops containing a fertility parameter $\tilde{f}_{1 j}$ in the set $\mathcal{L}_{\mathrm{f}}$. Then all loops $L \in \mathcal{L}_{\mathrm{f}}$ are pairwise connected and

$$
Q=-\sum_{\mathcal{L}_{\mathrm{f}}} L q(\breve{L})-1+\sum_{\mathcal{L}_{\mathrm{A}} \backslash \mathcal{L}_{\mathrm{f}}} L-\sum_{\left(\mathcal{L}_{\mathrm{A}} \backslash \mathcal{L}_{\mathrm{f}}\right)^{2 *}} L M+\sum_{\left(\mathcal{L}_{\mathrm{A}} \backslash \mathcal{L}_{\mathrm{f}}\right)^{3 *}} L M N-\cdots
$$

(cf. Eq. 15). In Rueffler (submitted) it is shown that

$$
0=-R_{0}^{-1} \sum_{\mathcal{L}_{\mathrm{f}}} L q(\breve{L})-1+\sum_{\mathcal{L}_{\mathrm{A}} \backslash \mathcal{L}_{\mathrm{f}}} L-\sum_{\left(\mathcal{L}_{\mathrm{A}} \backslash \mathcal{L}_{\mathrm{f}}\right)^{2 *}} L M+\sum_{\left(\mathcal{L}_{\mathrm{A}} \backslash \mathcal{L}_{\mathrm{f}}\right)^{3 *}} L M N-\cdots
$$

or, equivalently,

$$
R_{0}=\frac{\sum_{\mathcal{L}_{\mathrm{f}}} L q(\breve{L})}{1-\sum_{\mathcal{L}_{\mathrm{A}} \backslash \mathcal{L}_{\mathrm{f}}} L+\sum_{\left(\mathcal{L}_{\mathrm{A}} \backslash \mathcal{L}_{\mathrm{f}}\right)^{2 *}} L M-\sum_{\left(\mathcal{L}_{\mathrm{A}} \backslash \mathcal{L}_{\mathrm{f}}\right)^{3 *}} L M N+\cdots} .
$$


(a)

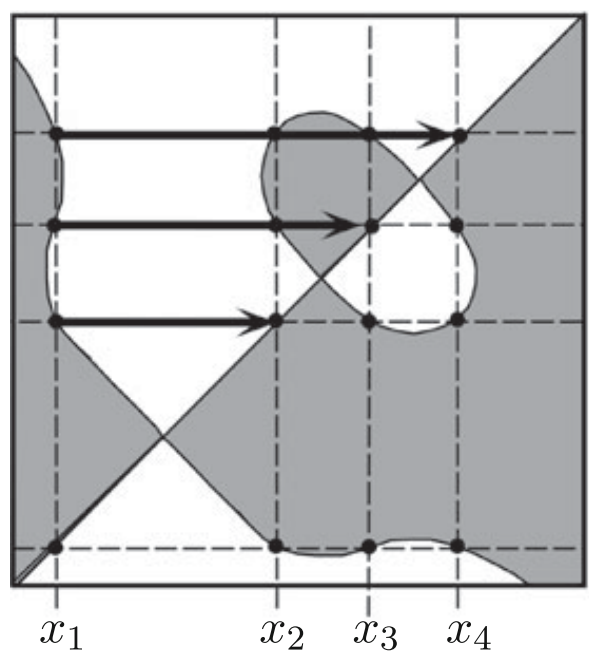

(b)

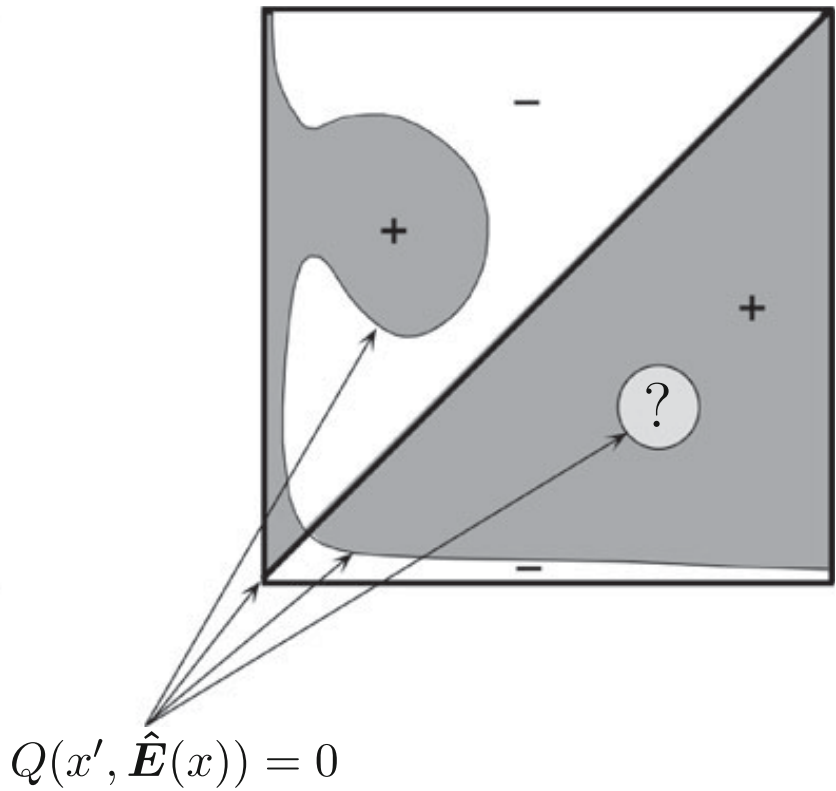

Fig. 8 Pairwise invadability plots with resident trait $x$ on the horizontal axis and mutant trait $x^{\prime}$ on the vertical axis. a A graphical representation of the necessary and sufficient conditions for the existence of an optimisation principle. The PIP should be (i) antisymmetric, i.e., flipping it over the main diagonal leads to the same picture except that plus becomes minus and vice versa, (ii) transitive, i.e., selectively equivalent resident traits, having zero invasion fitness with respect to each other, should have exactly the same pattern of plusses in the vertical direction (and hence also in the horizontal direction). Figure from Metz et al. (2008a). b The information given by the solution of $Q\left(x^{\prime}, \hat{\boldsymbol{E}}(x)\right)=0$ in the $\left(x, x^{\prime}\right)$-plane about the signs of the invasion fitness $\lambda_{\mathrm{d}}\left(x^{\prime}, \hat{\boldsymbol{E}}(x)\right)$. Figure from Metz and Leimar (2011)

With the definitions used in Eq. (15) we can write more compactly $R_{0}=-a / b$. We note that in this case $b=-Q_{\mathrm{S}}$, where $Q_{\mathrm{S}}$ is computed as in Eq. (5) but from the state-transition matrix $S$ instead of the full population projection matrix $A=F+S$.

Assume that $\psi$ is a candidate optimisation principle derived from $Q$. Here we prove that then for path connected trait spaces generically $\psi$ is indeed an optimisation principle.

First we observe that $\psi$ is an optimisation principle for some eco-evolutionary model if and only if $\psi$ is an optimisation principle for all models that we can derive from this model by restricting the traits to a one-dimensional manifold. Hence, it suffices to prove our claim for one-dimensional trait spaces. The advantage is that for such trait spaces we can fall back on the graphical tool of pairwise invadability plots (PIPs), i.e., sign plots of $\lambda_{\mathrm{d}}\left(x^{\prime}, \hat{\boldsymbol{E}}(x)\right)$ in the $\left(x, x^{\prime}\right)$-plane.

Figure 8 a shows necessary and sufficient conditions on a PIP for $\lambda_{\mathrm{d}}$ to support an optimisation principle. If the sign-plot of $Q$ would satisfy these conditions, then any $\psi$ derived from $Q$ would indeed be an optimisation principle if the sign plot of $Q$ were to coincide with the sign plot of $\lambda_{\mathrm{d}}$. We will argue that the conditions on these sign plots are such that if the sign plot for $Q$ satisfies them, generically the sign plot of $\lambda_{d}$ will coincide with it.

Figure $8 \mathrm{~b}$ shows the gist of the argument. In a path connected trait space a lot of information about the sign of $\lambda_{\mathrm{d}}$ can be derived from the sign of $Q$. Basically, all points that can be connected to points near the diagonal, where we know the signs of 
$\lambda_{\mathrm{d}}$ and $Q$ to coincide, and that do not pass a sign change of $Q$ have the same sign as the latter points. Moreover, from the arguments in Metz and Leimar (2011) we know that if along a path $Q$ changes sign from negative to positive $\lambda_{\mathrm{d}}$ follows suit. Only when the sign from $Q$ changes from positive to negative the sign of $\lambda_{\mathrm{d}}$ need not do so. Our argument will be that these considerations together with the strong coupling between different regions of the PIP imposed on $Q$ by the conditions from Fig. 8a make that generically $Q$ will not satisfy the latter unless there is a mechanistic reason which then also makes that generically the signs of $Q$ and $\lambda_{\mathrm{d}}$ coincide in full.

We start from a function $\psi$ that relates to the sign structure of $Q$ in the same way as an optimisation principle would relate to the sign structure of $\lambda_{d}$. Hence, the sign plot of $Q$ satisfies the necessary and sufficient conditions that for $\lambda_{\mathrm{d}}$ would imply that there is an optimisation principle. All areas with positive $Q$ are also areas where $\lambda_{\mathrm{d}}$ is positive. Since near the diagonal the sign of $Q$ is representative for that of $\lambda_{\mathrm{d}}$, also all areas with negative $Q$ that connect to the diagonal have negative $\lambda_{\mathrm{d}}$. The only case where the sign of $\lambda_{\mathrm{d}}$ need not match that of $Q$ is in areas with negative $Q$ that do not connect to the diagonal. However, the sign pattern of $Q$ satisfies the conditions from Fig $8 \mathrm{a}$, so any such area is matched exactly by a symmetrically placed area with positive $Q$, and moreover, is connected to still other negative and thereby to positive areas by the transitivity condition. Now assume that in the PIP that particular area with negative $Q$ does not exist. So $Q$ has a very precisely prescribed negative region that $\lambda_{\mathrm{d}}$ has not. The only way for $Q$ to have such a region is by another real solution of the characteristic equation changing sign precisely on the border of this region. Our contention is that, within model classes for which the sign of $Q$ has a pattern compatible with an optimisation principle while $\lambda_{\mathrm{d}}$ has not, most slight changes in the underlying eco-evolutionary model will spoil such a precise match in $Q$. Hence, generically such configurations do not occur. Of course, for general models, most slight changes will also spoil the existence of an optimisation principle, full stop. However, what is generic depends on the class of models under consideration. The methodology in this article concentrates on the mechanistic delimitation of classes of models all members of which have an optimisation principle, or in the present argument, for which $Q$ is compatible with such a principle. Hence, with genericity we mean here genericity within such classes and hence concentrate on slight changes that do not move the model out of its class.

\section{Appendix B: Proof of Proposition 16}

For the proof we show that with the assumptions of Proposition 16 we can write " $R_{0}$ " as

$$
\boldsymbol{u}_{1}(\hat{\boldsymbol{E}})\left(\mathrm{I}-\mathrm{M}_{1}(\hat{\boldsymbol{E}})\right)^{-1} \boldsymbol{v}_{1}(\hat{\boldsymbol{E}}) \times \boldsymbol{u}_{2}(\boldsymbol{x})\left(\mathrm{I}-\mathrm{M}_{2}(\boldsymbol{x})\right)^{-1} \boldsymbol{v}_{2}(\boldsymbol{x})
$$

repeated $n / 2$ times, where $\mathrm{M}_{i}$ are the within-class projection matrices, $\boldsymbol{u}_{i}$ are row vectors and $\boldsymbol{v}_{i}$ are column vectors. Each scalar $\boldsymbol{u}_{j}\left(\mathrm{I}-\mathrm{M}_{j}\right)^{-1} \boldsymbol{v}_{j}$ then depends either only on $\boldsymbol{x}$ or only on $\hat{\boldsymbol{E}}$ and " $R_{0}$ " can be written as a product of two functions, one 
being a function of $\boldsymbol{x}$ and the other of $\hat{\boldsymbol{E}}$. When read from right to left, Expression (B1) describes the flow of individuals through the life cycle. More specifically, each scalar $\boldsymbol{u}_{j}\left(\mathrm{I}-\mathrm{M}_{j}\right)^{-1} \boldsymbol{v}_{j}$ gives the number of descendants of an individual starting in the $j$ th obligatory i-state that enter the $j+1$ th obligatory i-state, where "descendants" includes survival of the focal individual. The matrix $\left(I-M_{j}\right)^{-1}$ is the limit of the geometric series generated by $\mathrm{M}_{j}$ and describes the flow of individual through the i-states of the $j$ class. For instance, Equation (20) in the main text can be written as

$$
\begin{aligned}
“ R_{0} "= & \left(0, \tilde{f}_{15}, \tilde{f}_{16}\right)\left(\begin{array}{ccc}
1 & 0 & 0 \\
\tilde{s}_{54} & 1 & 0 \\
\tilde{s}_{64} & 0 & 1
\end{array}\right)\left(\begin{array}{l}
1 \\
0 \\
0
\end{array}\right) \\
& \times\left(\tilde{s}_{41}, \tilde{s}_{42}, \tilde{s}_{43}\right)\left(\begin{array}{ccc}
\frac{1}{1-\tilde{s}_{11}} & 0 & 0 \\
\frac{\tilde{s}_{21}}{\left(1-\tilde{s}_{11}\right)\left(1-\tilde{s}_{22}\right)} & \frac{1}{1-\tilde{s}_{22}} & 0 \\
\frac{\tilde{s}_{31}}{\left(1-\tilde{s}_{11}\right)\left(1-\tilde{s}_{33}\right)} & 0 & \frac{1}{1-\tilde{s}_{33}}
\end{array}\right)\left(\begin{array}{l}
1 \\
0 \\
0
\end{array}\right),
\end{aligned}
$$

where the first three factors result in the term in the first bracket while the factors four to six result in the term in the second bracket. (Note that in this specific example " $R_{0}$ " actually equals the true $R_{0}$ because entering $G_{1}=\{1,2,3\}$ is only possible by passing through i-state 1 , the single birth state of the life cycle.)

The matrix $\left(I-M_{j}\right)$ is invertible if the dominant eigenvalue of $M_{j}$ is less than one. Consider for any $\boldsymbol{x}$ the corresponding feedback environment $\hat{\boldsymbol{E}}(\boldsymbol{x})$ where " $R_{0}$ " $=1$. This feedback environment exists by the assumption that the community models under consideration have equilibria. This implies that indeed all matrices $\mathrm{M}_{j}(\hat{\boldsymbol{E}})$ and $\mathrm{M}_{j}(\boldsymbol{x})$ have dominant eigenvalues less than one for else the feedback environment is such that there exist a class that can grow indefinitely. Now consider a rare mutant $\boldsymbol{x}^{\prime}$. Since $\mathrm{M}_{j}(\hat{\boldsymbol{E}})$ is not affected by the trait vector its eigenvalues are unchanged. Conversely, matrices $\mathrm{M}_{j}\left(\boldsymbol{x}^{\prime}\right)$ are not affected by the feedback environment. If such a matrix has a dominant eigenvalue larger than one this still holds true if that mutant would be a resident type. Thus, such mutants are excluded from our universe of discourse by the assumption of community dynamical equilibria. Note, that the problem that from a naive perspective the matrices $\mathrm{M}_{j}(\boldsymbol{x})$ could have eigenvalues larger than one is immediately ruled out when all its entries are state-transitions $\tilde{s}_{l k}$, or, phrased differently, if there are no within-class fertilities $\tilde{f}_{l k}$.

The conditions in Proposition 16 connect to Expression (B1) in the following way. The demographic parameters corresponding to arrows from a class with $k$ i-states to a class with $l$ i-states can be written in a $l \times k$-matrix. If the next class can only be entered in its first numbered i-state this matrix can be written as

$$
\left(\begin{array}{c}
1 \\
0 \\
\vdots \\
0
\end{array}\right)\left(u_{1}(z), \ldots, u_{k}(z)\right)
$$


where $z$ stands for $\boldsymbol{x}$ or $\hat{\boldsymbol{E}}$. Around the boundary between two classes we have

$$
\left(\mathrm{I}-\mathrm{M}_{1}(\boldsymbol{y})\right)^{-1}\left(\begin{array}{c}
1 \\
0 \\
\vdots \\
0
\end{array}\right)\left(u_{1}(z), \ldots, u_{k}(z)\right)\left(\mathrm{I}-\mathrm{M}_{2}(z)\right)^{-1}
$$

where $(\boldsymbol{y}, \boldsymbol{z})$ stands for $(\boldsymbol{x}, \hat{\boldsymbol{E}})$ or $(\hat{\boldsymbol{E}}, \boldsymbol{x})$. Thus, we indeed find the pattern described in Expression (B1).

Remark (I) states that under certain restrictions optimisation is also possible if the first class can only be left through the last numbered i-state in the class. Then the transition between two classes is give by

$$
\left(\begin{array}{c}
v_{1}(\boldsymbol{x}, \hat{\boldsymbol{E}}) \\
\vdots \\
v_{l}(\boldsymbol{x}, \hat{\boldsymbol{E}})
\end{array}\right)(0, \ldots, 0,1)
$$

The pattern of Expression (B1) can then be found under two different conditions:

1. Exit from a non-evolving class:

$$
\left(\mathrm{I}-\mathrm{M}_{1}(\boldsymbol{x})\right)^{-1}\left(\begin{array}{c}
v_{1}(\boldsymbol{x}, \hat{\boldsymbol{E}}) \\
\vdots \\
v_{l}(\boldsymbol{x}, \hat{\boldsymbol{E}})
\end{array}\right)(0, \ldots, 0,1)\left(\mathrm{I}-\mathrm{M}_{2}(\hat{\boldsymbol{E}})\right)^{-1}
$$

In this case it is necessary that $v_{1}$ to $v_{l}$ all are regulated in the same way (e.g., since they are all unregulated).

2. Exit from an unregulated class:

$$
\left(\mathrm{I}-\mathrm{M}_{1}(\hat{\boldsymbol{E}})\right)^{-1}\left(\begin{array}{c}
v_{1}(\boldsymbol{x}, \hat{\boldsymbol{E}}) \\
\vdots \\
v_{l}(\boldsymbol{x}, \hat{\boldsymbol{E}})
\end{array}\right)(0, \ldots, 0,1)\left(\mathrm{I}-\mathrm{M}_{2}(\boldsymbol{x})\right)^{-1}
$$

In this case $v_{1}$ to $v_{l}$ should not evolve.

\section{Appendix C: Relationship between allocation functions and trade-off curve}

Let us call the two allocation functions $\xi_{1}(\theta)=x_{1}$ and $\xi_{2}(\theta)=x_{2}$. In this appendix we prove that if both allocation functions are convex (concave), then the resulting trade-off function $x_{2}=T\left(x_{1}\right)=\xi_{2}\left(\xi_{1}^{(-1)}\left[x_{1}\right]\right)$ is also convex (concave). Without restriction of generality we assume that $\xi_{1}^{\prime}(\theta)>0$ and $\xi_{2}^{\prime}(\theta)<0$. Then the statement follows directly from 


$$
\frac{\mathrm{d}^{2} \xi_{2}\left(\xi_{1}^{(-1)}\left[x_{1}\right]\right)}{\mathrm{d} x_{1}^{2}}=\frac{\frac{\mathrm{d} \xi_{1}(\theta)}{\mathrm{d} \theta} \frac{\mathrm{d}^{2} \xi_{2}(\theta)}{\mathrm{d} \theta^{2}}-\frac{\mathrm{d} \xi_{2}(\theta)}{\mathrm{d} \theta} \frac{\mathrm{d}^{2} \xi_{1}(\theta)}{\mathrm{d} \theta^{2}}}{\left(\frac{\mathrm{d} \xi_{1}(\theta)}{\mathrm{d} \theta}\right)^{3}}
$$

\section{Appendix D: Curvature of the invasion boundaries}

In this appendix we prove Propositions 18. Invasion boundaries are characterized by functions $x_{1} \mapsto x_{2}=I\left(x_{1}\right)$ that are implicitly defined by $p\left(\left(x_{1}, I\left(x_{1}\right)\right), \hat{\boldsymbol{E}}\right)=0$, where $p$ can be any fitness proxy. In this and the following appendix we use the following notation. To indicate whether a specific loop contains a certain trait we add the traits that are contained in the loop as subscripts. For instance, the loop $L_{x_{i}}$ contains the trait $x_{i}$ and $L_{x_{i} x_{j}}$ contains both $x_{i}$ and $x_{j}$. For each set of traits the corresponding set of loops containing this combination of traits is indicated by adding the corresponding subscripts to $\mathcal{L}$, e.g., $\mathcal{L}_{x_{i} x_{j}}$. In particular, $\sum_{\mathcal{L}_{x_{i}}} L_{x_{i}}$ denotes the sum of all loops that contain a specific trait $x_{i}$. We use the following short hand for partial derivatives:

$$
\dot{L}_{x_{i}}:=\frac{\partial L_{x_{i}}}{\partial x_{i}}=\frac{L_{x_{i}}}{x_{i}} \quad \text { and } \quad \dot{L}_{x_{i} x_{j}}:=\frac{\partial^{2} L_{x_{i} x_{j}}}{\partial x_{i} \partial x_{j}}=\frac{L_{x_{i} x_{j}}}{x_{i} x_{j}}
$$

Throughout this appendix function $q(\alpha)$ is used as introduced in Sect. 2.2.

By definition

$$
Q\left(\left(x_{1}, I\left(x_{1}\right)\right), \hat{\boldsymbol{E}}\right)=0
$$

Let the operators $\partial_{1}$ and $\partial_{2}$ stand for differentiating for $x_{1}$ and $x_{2}=I\left(x_{1}\right)$. Differentiating twice for $x_{1}$ then gives

$$
\partial_{1}^{2} Q+2 \partial_{1} I \partial_{1} \partial_{2} Q+\partial_{2}^{2} Q\left(\partial_{1} I\right)^{2}+\partial_{2} Q \partial_{1}^{2} I=0
$$

After substituting $\partial_{1}^{2} Q=0=\partial_{2}^{2} Q$ (since $Q$ is linear in all traits separately) this gives

$$
\partial_{1}^{2} I=-\frac{2 \partial_{1} I \partial_{1} \partial_{2} Q}{\partial_{2} Q}
$$

with $\partial_{1} I<0$ and $\partial_{2} Q=-\sum_{\mathcal{L}_{x_{2}}} \dot{L}_{x_{2}} q\left(\breve{L}_{x_{2}}\right)>0$. The inequality follows from the fact that $Q$ is a fitness proxy and that $\lambda_{\mathrm{d}}$ is monotonically increasing in all demographic parameters. We can now treat the three cases of Proposition 18.

For case (i) we can substitute $\partial_{1} \partial_{2} Q=0$ and it follows that invasion boundaries are linear.

For case (ii) we can substitute

$$
\partial_{1} \partial_{2} Q=-\sum_{\mathcal{L}_{x_{1} x_{2}}} \dot{L}_{x_{1} x_{2}} q\left(\breve{L}_{x_{1} x_{2}}\right)>0
$$

Hence, the invasion boundaries have positive curvature and are convex. 
For case (iii) we can substitute

$$
\partial_{1} \partial_{2} Q=\sum_{\mathcal{L}_{x_{1}}{\stackrel{*}{\times} \mathcal{L}_{x_{2}}}} \dot{L}_{x_{1}} \dot{L}_{x_{2}} q\left(\breve{L}_{x_{1}} \cup \breve{L}_{x_{2}}\right)<0,
$$

where $\mathcal{L}_{x_{1}} \times \mathcal{L}_{x_{2}}$ denotes the Cartesian product over the two sets of loops $\mathcal{L}_{x_{1}}$ and $\mathcal{L}_{x_{2}}$. The star indicates that pairs of loops that are not unconnected are excluded from the Cartesian product. Therefore the invasion boundaries have negative curvature and are concave.

\section{Appendix E: Curvature of the invasion boundaries in higher dimensions}

We first prove that $\partial T / \partial x_{j}<0$ for all $j \in\{j, \ldots, m\}$ if and only if at any point $\boldsymbol{x}$ on the trade-off manifold all vectors orthogonal to the tangent plane can be chosen to point into the interior of the positive cone. Let $\boldsymbol{x}^{*}$ be a point on the trade-off manifold. Assume that the vectors orthogonal to the tangent plane of the trade-off manifold at $\boldsymbol{x}^{*}$ can all be chosen so as to point into the interior of the positive cone. This tangent plane consists of all vectors

$\boldsymbol{y}=\left(\left(x_{1}^{*}-x_{1}\right), \ldots,\left(x_{m-1}^{*}-x_{m-1}\right), \sum_{i=1}^{m-1} \frac{\partial T}{\partial x_{i}}\left(x_{i}^{*}-x_{i}\right)\right)$ for some $\left(x_{1}, \ldots, x_{m-1}\right)$.

Because of our assumption there exists a positive normal vector $\boldsymbol{n}$ to the tangent manifold. Then

$$
\boldsymbol{n} \cdot \boldsymbol{y}=n_{1}\left(x_{1}^{*}-x_{1}\right)+\cdots n_{m-1}\left(x_{m-1}^{*}-x_{m-1}\right)+n_{m} \sum_{i=1}^{m-1} \frac{\partial T}{\partial x_{i}}\left(x_{i}^{*}-x_{i}\right)=0 .
$$

By choosing $x_{i} \neq x_{i}^{*}$ and $x_{j}=x_{j}^{*}$ for all $j \neq i$ it follows $\partial T / \partial x_{i}<0$. Conversely, by choosing $n_{m}>0, x_{i} \neq x_{i}^{*}$ and $x_{j}=x_{j}^{*}$ it follows from $\partial T / \partial x_{i}<0$ that $n_{i}>0$.

Next we show that a singular point $x^{*}$ is locally uninvadable if and only if $I \stackrel{*}{\succ} T$ where

$$
I \stackrel{*}{\succ} T: \Longleftrightarrow(\mathrm{H}(I)-\mathrm{H}(T))\left(x_{1}^{*}, \ldots, x_{m-1}^{*}\right) \text { positive definite, }
$$

and with $\mathrm{H}(I)$ and $\mathrm{H}(T)$ denoting the Hessian matrix of the functions $I\left(x_{1}, \ldots, x_{m-1}\right)$ and $T\left(x_{1}, \ldots, x_{m-1}\right)$, respectively. The expression $(\mathrm{H}(I)-\mathrm{H}(T))\left(x_{1}^{*}, \ldots, x_{m-1}^{*}\right)$ describes the curvature of the manifold that results when $T\left(x_{1}, \ldots, x_{m-1}\right)$ is subtracted from $I\left(x_{1}, \ldots, x_{m-1}\right)$ locally around the point of tangency. If this manifold is convex, then locally around the singular point, except for the point of tangency, $I$ lies outside the feasibility set delineated by $T$ and the singular point is uninvadable. And indeed, any manifold $U$ is locally around a point $\boldsymbol{x}^{*}$ convex if the Hessian matrix $\mathrm{H}\left(U\left(x_{1}^{*}, \ldots, x_{m-1}^{*}\right)\right)=\left[h_{i j}\right]$ is positive definite. 
We call a singular point $\boldsymbol{x}^{*}=\left(x_{1}^{*}, \ldots, x_{m}^{*}\right)$ symmetric if

(a) $\left.\frac{\partial T}{\partial x_{1}}\right|_{*}=\cdots=\left.\frac{\partial T}{\partial x_{m-1}}\right|_{*}=-1$

(b) $Q\left(\boldsymbol{x}^{\prime}, \hat{\boldsymbol{E}}\left(\boldsymbol{x}^{*}\right)\right)=Q\left(\sigma\left(\boldsymbol{x}^{\prime}\right), \hat{\boldsymbol{E}}\left(\boldsymbol{x}^{*}\right)\right)$ for any permutation $\sigma$ of $\boldsymbol{x}^{\prime}$.

We now prove Propositions 19 and 20. We use the same notation as introduced in the previous appendix. By focusing on the traits $x_{i}, x_{j}$ and $x_{m}=I\left(x_{i}, x_{j}, A O A\right)$ with $i \neq j, i \neq m$ and $j \neq m$, we can write

$$
Q\left(\left(x_{i}, x_{j}, I\left(x_{i}, x_{j}, A O A\right), A O A\right), \hat{\boldsymbol{E}}\right)=0,
$$

where $A O A$ stands for all other arguments. The operators $\partial_{i}, \partial_{j}$ and $\partial_{m}$ stand for differentiating for $x_{i}, x_{j}$ and $x_{m}=I\left(x_{i}, x_{j}, A O A\right)$. Differentiating for $x_{i}$ then gives

$$
\partial_{i} Q+\partial_{m} Q \partial_{i} I=0
$$

Subsequently differentiating for $x_{j}$ gives

$$
\partial_{i} \partial_{j} Q+\partial_{i} \partial_{m} Q \partial_{j} I+\partial_{j} \partial_{m} Q \partial_{i} I+\partial_{m}^{2} Q \partial_{i} I \partial_{j} I+\partial_{m} Q \partial_{i} \partial_{j} I=0
$$

Using that $\partial_{m}^{2} Q=0$, which follows from the fact that $Q$ is linear in all traits separately, and solving for $\partial_{i} \partial_{j} I$ we get

$$
\partial_{i} \partial_{j} I=\frac{-\partial_{i} \partial_{j} Q-\partial_{i} \partial_{m} Q \partial_{j} I-\partial_{j} \partial_{m} Q \partial_{i} I}{\partial_{m} Q} .
$$

If all evolving loops are single-trait loops and if no pair of evolving loops exist that is unconnected, then $\partial_{k} \partial_{l} Q=0$. Thus, $\partial_{i} \partial_{j} I=0$ and invasion boundaries are linear. This proves Proposition 19.

Evaluating at a symmetric singular point, we can replace $\partial_{i} I$ and $\partial_{j} I$ with -1 (since at the singular point the graph of $I$ is tangent to the trade-off manifold). Then Eq. (E1) simplifies to

$$
\partial_{i} \partial_{j} I\left(x_{i}^{*}, x_{j}^{*}, A O A^{*}\right)=\frac{\partial_{i} \partial_{m} Q+\partial_{j} \partial_{m} Q-\partial_{i} \partial_{j} Q}{\partial_{m} Q} .
$$

To find the double derivative $\partial_{i}^{2} I\left(x_{i}^{*}, x_{j}^{*}, A O A^{*}\right)$ we substitute $i=j$ in Eq. (E2) which gives

$$
\partial_{i}^{2} I\left(x_{i}^{*}, x_{j}^{*}, A O A^{*}\right)=\frac{2 \partial_{i} \partial_{m} Q}{\partial_{m} Q}
$$

with $\partial_{m} Q=-\sum_{\mathcal{L}_{x_{m}}} \dot{L}_{x_{m}} q\left(\breve{L}_{x_{m}}\right)>0$. The inequality follows from the fact that $Q$ is a fitness proxy and that $\lambda_{\mathrm{d}}$ is monotonically increasing in all demographic parameters. 
A Hessian matrix $\mathrm{H}$ is positive definite if and only if $h_{i j}^{2}<h_{i i} h_{j j}$ and if $h_{i i}>0$ for all $i, j$, since any positive definite matrix can be seen as a co-variance matrix and vice versa. Conversely, a Hessian matrix $\mathrm{H}$ is negative definite if and only if for the entries of the matrix $\mathrm{G}:=-\mathrm{H}$ we have $g_{i j}^{2}<g_{i i} g_{j j}$ for all $i, j$.

If at least one multi-trait loop exists and all pairs of evolving loops are connected, then

$$
\partial_{k} \partial_{l} Q=-\sum_{\mathcal{L}_{x_{k} x_{l}}} \dot{L}_{x_{k} x_{l}} q\left(\breve{L}_{x_{k} x_{l}}\right)
$$

Thus, the Hessian matrix of the invasion boundary at a singular point is positive definite if for all $i, j \in\{1, \ldots, m-1\}$

$$
a^{2}<4 b c
$$

where

$$
\begin{aligned}
a & =-\sum_{\mathcal{L}_{x_{i} x_{m}}} \dot{L}_{x_{i} x_{m}} q\left(\breve{L}_{x_{i} x_{m}}\right)-\sum_{\mathcal{L}_{x_{j} x_{m}}} \dot{L}_{x_{j} x_{m}} q\left(\breve{L}_{x_{j} x_{m}}\right)+\sum_{\mathcal{L}_{x_{i} x_{j}}} \dot{L}_{x_{i} x_{j}} q\left(\breve{L}_{x_{i} x_{j}}\right) \\
b & =-\sum_{\mathcal{L}_{x_{i} x_{m}}} \dot{L}_{x_{i} x_{m}} q\left(\breve{L}_{x_{i} x_{m}}\right) \\
c & =-\sum_{\mathcal{L}_{x_{j} x_{m}}} \dot{L}_{x_{j} x_{m}} q\left(\breve{L}_{x_{j} x_{m}}\right)
\end{aligned}
$$

Under the assumption that at least one multi-trait loop exists and all pairs of evolving loops are connected we can write $Q$ as

$$
\begin{aligned}
Q= & -1+\sum_{i=1}^{m} x_{i} \sum_{\mathcal{L}_{x_{i}}} \dot{L}_{x_{i}} q\left(\breve{L}_{x_{i}}\right)+\sum_{\substack{i, j), i \neq j \\
i, j \in\{1, \ldots, m\}}} x_{i} x_{j} \sum_{\mathcal{L}_{x_{i} x_{j}}} \dot{L}_{x_{i} x_{j}} q\left(\breve{L}_{x_{i} x_{j}}\right) \\
& +\sum_{\substack{(i, j, k) \\
i \neq j, j \neq k, i \neq k \\
i, j, k \in\{1, \ldots, m\}}} x_{i} x_{j} x_{k} \sum_{\mathcal{L}_{x_{i} x_{j} x_{k}}} \dot{L}_{x_{i} x_{j} x_{k}} q\left(\breve{L}_{x_{i} x_{j} x_{k}}\right)+\ldots,
\end{aligned}
$$

where no function $q$ contains a trait. The symmetry $Q\left(\boldsymbol{x}^{\prime}, \hat{\boldsymbol{E}}\left(\boldsymbol{x}^{*}\right)\right)=Q\left(\sigma\left(\boldsymbol{x}^{\prime}\right), \hat{\boldsymbol{E}}\left(\boldsymbol{x}^{*}\right)\right)$ implies equality of the each sum over traits under a permutation $\sigma\left(\boldsymbol{x}^{\prime}\right)$ since these terms lie in independent subspaces of the space of polynomials. In particular,

$$
\sum_{\mathcal{L}_{x_{i} x_{j}}} \dot{L}_{x_{i} x_{j}} q\left(\breve{L}_{x_{i} x_{j}}\right)=\sum_{\mathcal{L}_{x_{k} x_{l}}} \dot{L}_{x_{k} x_{l}} q\left(\breve{L}_{x_{k} x_{l}}\right)
$$


Thus, $a=b=c$ which implies that Inequality (E4) is fulfilled. Hence, $\mathrm{H}$ is positive definite and invasion boundaries are convex. This completes the proof of Proposition 20(i).

If all evolving loops are single-trait loops and at least one pair of evolving loops exist that is unconnected, then

$$
\partial_{k} \partial_{l} Q=-\sum_{\mathcal{L}_{x_{k}}{ }^{*} \mathcal{L}_{x_{l}}} \dot{L}_{x_{k}} \dot{L}_{x_{l}} q\left(\breve{L}_{x_{k}} \cup \breve{L}_{x_{l}}\right),
$$

and it follows that

$$
\partial_{i}^{2} I=-\frac{2 \sum_{\mathcal{L}_{x_{i}}{ }^{*} \mathcal{L}_{x_{m}}} \dot{L}_{x_{i}} \dot{L}_{x_{m}} q\left(\breve{L}_{x_{i}} \cup \breve{L}_{x_{m}}\right)}{\sum_{\mathcal{L}_{x_{m}}} \dot{L}_{x_{m}} q\left(\breve{L}_{x_{m}}\right)}
$$

and

$$
\partial_{i} \partial_{j} I=-\frac{\sum_{\mathcal{L}_{x_{i}} \times \mathcal{L}_{x_{m}}} \dot{L}_{x_{i}} \dot{L}_{x_{m}} q\left(\breve{L}_{x_{i}} \cup \breve{L}_{x_{m}}\right)+\sum_{\mathcal{L}_{x_{j}} \times \mathcal{L}_{x_{m}}} \dot{L}_{x_{j}} \dot{L}_{x_{m}} q\left(\breve{L}_{x_{j}} \cup \breve{L}_{x_{m}}\right)}{-\sum_{\mathcal{L}_{x_{i}}{ }^{*} \mathcal{L}_{x_{j}}} \dot{L}_{x_{i}} \dot{L}_{x_{j}} q\left(\breve{L}_{x_{i}} \cup \breve{L}_{x_{j}}\right)} \underbrace{}_{\sum_{\mathcal{L}_{x_{m}}} \dot{L}_{x_{m}} q\left(\breve{L}_{x_{m}}\right)} .
$$

From this we see that $\partial_{i}^{2} I<0$ and $\mathrm{H}$ cannot be positive definite because its diagonal entries are negative. However, $-\mathrm{H}$ is positive definite at a singular point if for all $i, j \in\{1, \ldots, m-1\}$

$$
a^{2}<4 b c
$$

where

$$
\begin{aligned}
a= & -\sum_{\mathcal{L}_{x_{i}} \times \mathcal{L}_{x_{m}}} \dot{L}_{x_{i}} \dot{L}_{x_{m}} q\left(\breve{L}_{x_{i}} \cup \breve{L}_{x_{m}}\right)-\sum_{\mathcal{L}_{x_{j}}{ }^{*} \mathcal{L}_{x_{m}}} \dot{L}_{x_{j}} \dot{L}_{x_{m}} q\left(\breve{L}_{x_{j}} \cup \breve{L}_{x_{m}}\right) \\
& +\sum_{\mathcal{L}_{x_{i}}{ }^{*} \mathcal{L}_{x_{j}}} \dot{L}_{x_{i}} \dot{L}_{x_{j}} q\left(\breve{L}_{x_{i}} \cup \breve{L}_{x_{j}}\right) \\
b=- & \sum_{\mathcal{L}_{x_{i}} \stackrel{*}{\times} \mathcal{L}_{x_{m}}} \dot{L}_{x_{i}} \dot{L}_{x_{m}} q\left(\breve{L}_{x_{i}} \cup \breve{L}_{x_{m}}\right) \\
c= & -\sum_{\mathcal{L}_{x_{j}}{ }_{*}^{\times} \mathcal{L}_{x_{m}}} \dot{L}_{x_{j}} \dot{L}_{x_{m}} q\left(\breve{L}_{x_{j}} \cup \breve{L}_{x_{m}}\right) .
\end{aligned}
$$

By the same argument as above, the symmetry $Q\left(\boldsymbol{x}^{\prime}, \hat{\boldsymbol{E}}\left(\boldsymbol{x}^{*}\right)\right)=Q\left(\sigma\left(\boldsymbol{x}^{\prime}\right), \hat{\boldsymbol{E}}\left(\boldsymbol{x}^{*}\right)\right)$ implies 


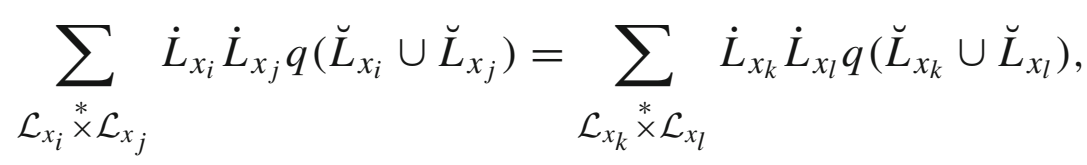

Thus, $a=b=c$ which implies that Inequality (E5) is fulfilled. Hence, $\mathrm{H}$ is negative definite and invasion boundaries are concave. This completes the proof of Proposition 20(ii).

\section{References}

Abrams PA (2005) 'Adaptive Dynamics’ vs. 'adaptive dynamics'. J Evol Biol 18:1162-1165

Armstrong RA, McGehee R (1980) Competitive exclusion. Am Nat 115:151-170

Bowers RG (2010) On the determination of evolutionary outcomes directly from the population dynamics of the resident. J Math Biol 62:901-924

Bowers RG, Hoyle A, White A, Boots M (2005) The geometric theory of adaptive evolution: trade-off and invasion plots. J Theor Biol 233:363-377

Brault S, Caswell H (1993) Pod-specific demography of killer whales (Orcinus orca). Ecology 74:14441454

Caswell H (1982) Optimal life histories and the maximization of reproductive value-a general theorem for complex life-cycles. Ecology 63:1218-1222

Caswell H (2001) Matrix population models, 2nd edn. Sinauer, Sunderland

Charlesworth B (1994) Evolution in age-structured populations, 2nd edn. Cambridge University Press, Cambridge

Charnov EL (1993) Life history invariants: some explorations of symmetry in evolutionary ecology. Oxford University Press, Oxford

de Mazancourt C, Dieckmann U (2004) Trade-off geometries and frequency-dependent selection. American Nat 164:765-778

Dercole F, Ferrière R, Rinaldi S (2002) Ecological bistability and evolutionary reversals under asymmetrical competition. Evolution 56:1081-1090

Dercole F, Rinaldi S (2008) Analysis of evolutionary processes: the adaptive dynamics approach and its applications. Princeton University Press, Princeton

Dieckmann U, Law R (1996) The dynamical theory of coevolution: A derivation from stochastic ecological processes. J Math Biol 34:579-612

Dieckmann U, Metz JAJ (2006) Surprising evolutionary predictions from enhanced ecological realism. Theor Popul Biol 69:263-381

Diekmann O (2004) A beginners guide to adaptive dynamics. In: Rudnicki R (ed) Mathematical modelling of population dynamics. Banach Center Publications, vol 63. Polish Academy of Sciences, Warszawa pp 47-86

Diekmann O, Gyllenberg M, Metz JAJ, Thieme HR (1998) On the formulation and analysis of general deterministic structured population models. I. Linear theory. J Math Biol 36:349-388

Diekmann O, Gyllenberg M, Huang H, Kirkilionis M, Metz JAJ, Thieme HR (2001) On the formulation and analysis of general deterministic structured population models. II. Nonlinear theory. J Math Biol 43:157-189

Diekmann O, Gyllenberg M, Metz JAJ (2003) Steady state analysis of structured population models. Theor Popul Biol 63:309-338

Ellner S, Hairston NG (1994) Role of overlapping generations in maintaining genetic variation in a fluctuating environment. Am Nat 143:403-417

Geritz SAH (2005) Resident-invader dynamics and the coexistence of similar strategies. J Math Biol 50:6782

Geritz SAH, Gyllenberg M, Jacobs FJA, Parvinen K (2002) Invasion dynamics and attractor inheritance. J Math Biol 44:548-560

Geritz SAH, Kisdi É, Meszéna G, Metz JAJ (1998) Evolutionarily singular strategies and the adaptive growth and branching of the evolutionary tree. Evol Ecol 12:35-57

Gyllenberg M, Service R (2011) Necessary and sufficient conditions for the existence of an optimisation principle in evolution. J Math Biol 62:359-369 
Heino M, Metz JAJ, Kaitala V (1998) The enigma of frequency-dependent selection. Trends Ecol Evol 13:367-370

Horn R, Johnson C (1985) Matrix analysis. Cambridge University Press, Cambridge

Hubbell S, Werner P (1979) Measuring the intrinsic rate of increase of populations with heterogeneous life-histories. Am Nat 113:277-293

Leimar O (2009) Multidimensional convergence stability. Evol Ecol Res 11:191-208

Levins SA (1970) Community equilibria and stability, and an extension of the competitive exclusion principle. Am Nat 104:413-423

Levins R (1962) Theory of fitness in a heterogeneous environment. I. The fitness set and the adaptive function. Am Nat 96:361-373

Levins R (1968) Evolution in changing environments. Princeton University Press, Princeton

MacArthur RH (1970) Species packing and competitive equilibrium for many species. Theor Popul Biol $1: 1-11$

Meszéna G, Gyllenberg M, Jacobs FJA, Metz JAJ (2005) Dynamics of similar populations: the link between population dynamics and evolution. Phys Rev Lett 95:078105(4)

Meszéna G, Gyllenberg M, Pásztor L, Metz JAJ (2006) Competitive exclusion and limiting similarity: a unified theory. Theor Popul Biol 69:68-87

Metz JAJ (2005) Eight personal rules for doing science. J Evol Biol 18:1178-1181

Metz JAJ (2008) Fitness. In: Jørgensen S, Fath B (eds) Evolutionary ecology. Encyclopedia of Ecology, vol [2]. Elsevier, pp 1599-1612

Metz JAJ (2011) Thoughts on the geometry of meso-evolution: collecting mathematical elements for a post-modern synthesis. In: Chalub FACC, Rodrigues JF (eds) The mathematics of Darwin's Legacy. Birkhauser, Basel

Metz JAJ, Diekmann O (1986) The dynamics of physiologically structured populations. Lecture Notes in Biomathematics, vol 68. Springer, Berlin

Metz JAJ, Geritz SAH, Meszéna G, Jacobs FJA, Van Heerwaarden JS (1996a) Adaptive dynamics: a geometrical study of the consequences of nearly faithful reproduction. In: van Strien S, Verduyn Lunel S (eds) Stochastic and spatial structures of dynamical systems, Proceedings of the Royal Dutch Academy of Science. North Holland, Dordrecht, Netherlands, pp 183-231. http://www.iiasa.ac.at/Research/ADN/ Series.html

Metz JAJ, Klinkhamer PGL, de Jong TJ (2009) A different model to explain delayed germination. Evol Ecol Res 11:177-190

Metz JAJ, Leimar O (2011) A simple fitness proxy for ESS calculations in structured populations with continuous traits, with applications to the evolution of haplo-diploids and genetic dimorphisms. J Biol Dyn 5:163-190

Metz JAJ, Mylius SD, Diekmann O (1996b) When does evolution optimize? On the relation between types of density dependence and evolutionarily stable life history parameters. IIASA working paper WP-96-04. http://www.iiasa.ac.at/Research/ADN/Series.html

Metz JAJ, Mylius SD, Diekmann O (2008) When does evolution optimise. Evol Ecol Res 10:629-654

Metz JAJ, Mylius SD, Diekmann O (2008) Even in the odd cases when evolution optimises, unrelated population dynamical details may shine through in the ESS. Evol Ecol Res 10:655-666

Metz JAJ, Nisbet RM, Geritz SAH (1992) How should we define 'fitness' for general ecological scenarios? Trends Ecol Evol 7:198-202

Mylius SD, Diekmann O (1995) On evolutionary stable life histories, optimisation and the need to be specific about density dependence. Oikos 74:218-224

Nowak M (1990) An evolutionary stable strategy may be inaccessible. J Theor Biol 142:237-241

Otto SP, Day T (2007) A biologist's guide to mathematical modeling in ecology and evolution. Princeton University Press, Princeton

Pásztor L, Meszéna G, Kisdi É (1996) $R_{0}$ or $r$ : a matter of taste. J Evol Biol 1996:511-518

Powell EO (1958) Criteria for the growth of contaminants and mutants in continuous culture. J Gen Microbiol 18:259-268

Ravigné V, Dieckmann U, Olivieri I (2009) Live where you thrive: joint evolution of habitat choice and local adaptation facilitates specialization and promotes diversity. Am Nat 174:E141-E169

Roff D (2002) Life history evolution. Sinauer, Sunderland

Rueffler C (submitted) A new formula for the basic reproduction ratio $R_{0}$. Bull Math Biol

Rueffler C, Van Dooren TJM, Leimar O, Abrams PA (2006) Disruptive selection and then what? Trends Ecol Evol 21:238-245 
Rueffler C, Van Dooren TJM, Metz JAJ (2004) Adaptive walks on changing landscapes: Levins' approached extended. Theor Popul Biol 65:165-178

Schneider K (2006) A multilocus-multiallele analysis of frequency-dependent selection induced by intraspecific competition. J Math Biol 52:483-523

Stearns SC (1992) The evolution of life histories. Oxford University Press, Oxford

Takada T, Nakajima H (1992) An analysis of life history evolution in terms of the density-dependent Lefkovitch matrix model. Math Biosci 112:155-176

Takada T, Nakajima H (1996) The optimal allocation for seed reproduction and vegetative reproduction in perennial plants: an application to the density-dependent transition matrix model. $\mathrm{J}$ Theor Biol 182:179-191

Takada T, Nakajima H (1998) Theorems on the invasion process in stage-structured populations with density-dependent dynamics. J Math Biol 36:497-514

Van Dooren TJM (2006) Protected polymorphism and evolutionary stability in pleiotropic models with trait-specific dominance. Evolution 60:1991-2003

Van Dooren TJM (2012) Adaptive dynamics for mendelian genetics. In: Metz JAJ, Dieckmann U (eds) Elements of adaptive dynamics. Cambridge University Press, Cambridge (in press) 